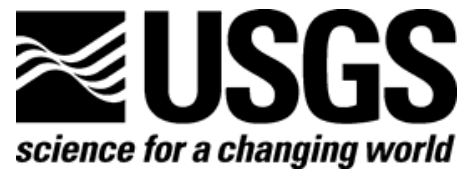

\title{
Occurrences of Calcareous Nannofossil, Dinoflagellate Cyst, and Pollen Taxa in Paleocene Strate in South Carolina
}

By Norman O. Frederiksen, Lucy E. Edwards, Laurel M. Bybell, and Jean M. Self-Trail

Report Series 2006-1353

U.S. Department of the Interior U.S. Geological Survey 


\section{U.S. Department of the Interior DIRK KEMPTHORNE, Secretary}

\section{U.S. Geological Survey \\ Mark D. Myers, Director}

U.S. Geological Survey, Reston, Virginia 2006

For product and ordering information:

World Wide Web: http://www.usgs.gov/pubprod

Telephone: 1-888-ASK-USGS

For more information on the USGS - the Federal source for science about the Earth, its natural and living resources, natural hazards, and the environment:

World Wide Web: http://www.usgs.gov

Telephone: 1-888-ASK-USGS

Suggested citation:

Frederiksen, Norman 0., Edwards, Lucy E., Bybell, Laurel M., Self-Trail, Jean M., 2006, Occurrences of Calcareous Nannofossil, Dinoflagellate Cyst, and Pollen Taxa in Paleocene Strata in South Carolina: Reston, Virginia, Open-File Report 2006-1353, p. \#\#; information on how to obtain if it's not from the group above.

Any use of trade, product, or firm names is for descriptive purposes only and does not imply endorsement by the U.S. Government.

Although this report is in the public domain, permission must be secured from the individual copyright owners to reproduce any copyrighted material contained within this report. 


\section{Contents}

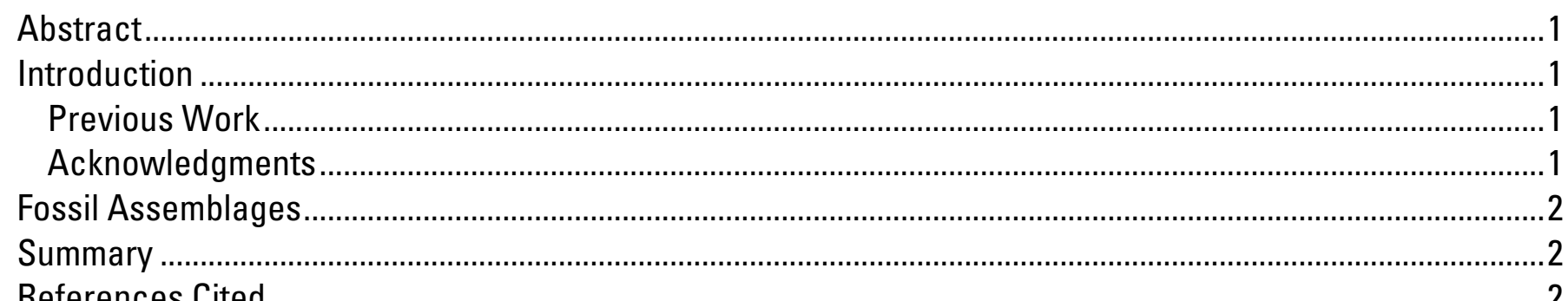

\section{Figures}

Figure 1. Index map of South Carolina showing the locations (black circles) and names of boreholes from which conventional cores, sidewall cores, or cuttings were recovered that were studied for this report, and the location of the Savannah River Site (hatched area).

Figure 2. Generalized comparison of nomenclature used by several authors for Paleocene geologic units in South Carolina. Shaded areas indicate missing stratigraphic sections. Fm., formation. Columns 4-7 are slightly modified from Falls and Prowell (2001); in column 5, formations are updip units; in column 7, formations are downdip units.

Figure 3. Distribution of dinocyst taxa in Paleocene samples from DNE/DOE corehole C10-A (ALL-348), Allendale County, South Carolina. $X=$ present; ? = questionable occurrence.

Figure 4. Distribution of dinocyst taxa in Paleocene cuttings samples from the Hilton Head water test borehole (BFT-2055), Beaufort County, South Carolina

Figure 5. Distribution of dinocyst taxa in Paleocene samples from the USGS/DNR St. Stephen corehole (BRK-644), Berkeley County, South Carolina.

Figure 6. Distribution of calcareous nannofossil taxa in Paleocene samples from the USGS/DNR St.

Stephen corehole (BRK-644), Berkeley County, South Carolina...

Figure 7. Distribution of pollen taxa in Paleocene samples from the USGS/DNR St. Stephen corehole (BRK-

644), Berkeley County, South Carolina.

Figure 8. Distribution of dinocyst taxa in Paleocene samples from the SRS P21TA corehole (BRN-335),

Barnwell County, South Carolina.

Figure 9. Distribution of pollen taxa in Paleocene samples from the SRS P21TA corehole (BRN-335),

Barnwell County, South Carolina.

Figure 10. Distribution of calcareous nannofossil taxa in Paleocene samples from the USGS Cannon Park corehole (CHN-800), Charleston County, South Carolina. 16

Figure 11. Distribution of calcareous nannofossil taxa in Paleocene samples from the USGS Moores Landing corehole (CHN-802), Charleston County, South Carolina.

Figure 12. Distribution of calcareous nannofossil taxa in Paleocene samples from the USGS Santee Coastal Reserve corehole (CHN-803), Charleston County, South Carolina.

Figure 13. Distribution of dinocyst taxa in Paleocene samples from the USGS/DNR Edisto Island corehole (COL-364), Colleton County, South Carolina.

Figure 14. Distribution of calcareous nannofossil taxa in Paleocene samples from the USGS/DNR Edisto Island corehole (COL-364), Colleton County, South Carolina. 
Figure 15. Distribution of pollen taxa in Paleocene samples from the USGS/DNR Edisto Island corehole (COL-364), Colleton County, South Carolina.

Figure 16. Distribution of dinocyst taxa in Paleocene samples from the USGS Clubhouse Crossroads No. 1 corehole (DOR-37), Dorchester County, South Carolina.

Figure 17. Distribution of calcareous nannofossil taxa in Paleocene samples from the USGS Clubhouse Crossroads No. 1 corehole (DOR-37), Dorchester County, South Carolina...

Figure 18. Distribution of pollen taxa in Paleocene samples from the USGS Clubhouse Crossroads No. 1 corehole (DOR-37), Dorchester County, South Carolina.

Figure 19. Distribution of calcareous nannofossil taxa in Paleocene samples from the USGS Pregnall corehole (DOR-208), Dorchester County, South Carolina.

Figure 20. Distribution of dinocyst taxa in Paleocene samples from the USGS St. George corehole (DOR211), Dorchester County, South Carolina.

Figure 21. Distribution of calcareous nannofossil taxa in Paleocene samples from the USGS St. George corehole (DOR-211), Dorchester County, South Carolina.

Figure 22. Distribution of calcareous nannofossil taxa in Paleocene sidewall-core samples from the DNR/DOE C-15 borehole (JAS-426), Jasper County, South Carolina.

Figure 23. Distribution of dinocyst taxa in Paleocene samples from the USGS/DNR Swansea corehole (LEX-844), Lexington County, South Carolina.

Figure 24. Distribution of pollen taxa in Paleocene samples from the USGS/DNR Swansea corehole (LEX844), Lexington County, South Carolina.

Figure 25. Distribution of dinocyst taxa in Paleocene samples from the USGS/DNR Clark School No. 1 corehole (ORG-393), Orangeburg County, South Carolina.

Figure 26. Distribution of pollen taxa in Paleocene samples from the USGS/DNR Clark School No. 1 corehole (ORG-393), Orangeburg County, South Carolina.

Figure 27. Distribution of dinocyst taxa in Paleocene samples from the USGS/DNR Clark School No. 2 corehole (ORG-430), Orangeburg County, South Carolina.

Figure 28. Distribution of pollen taxa in Paleocene samples from the USGS/DNR Clark School No. 2 corehole (ORG-430), Orangeburg County, South Carolina.

\section{Tables}

Table 1. South Carolina boreholes for which Paleocene dinoflagellate cyst, calcareous nannofossil, and pollen occurrence data are presented in this report and (or) have been presented in previous

U. S. Geological Survey open-file reports. 


\title{
Occurrences of Calcareous Nannofossil, Dinoflagellate Cyst, and Pollen Taxa in Paleocene Strate in South Carolina
}

\author{
By Norman 0. Frederiksen, Lucy E. Edwards, Laurel M. Bybell, and Jean M. Self-Trail
}

\begin{abstract}
This report presents 26 diagrams showing the occurrences of fossil dinocyst, calcareous nannofossil, and pollen assemblages in Paleocene samples from 15 boreholes in South Carolina.
\end{abstract}

\section{Introduction}

\section{Previous Work}

Paleocene stratigraphic units of South Carolina are not known in detail because most research on these units was based on discontinuous outcrop sections. Except for the many coreholes drilled at the Savannah River Site (SRS, fig. 1), few Paleocene cores were taken in South Carolina until the 1980's. Therefore, except at the SRS (Fallaw and Price, 1995, and references therein; Hargrove and Engelhardt, 1997), relatively little is known about the nature of the Paleocene stratigraphic units in the subsurface of South Carolina, and the Paleocene section is divided into only a few formations (fig. 2). Furthermore, until relatively recently, few paleontological studies of Paleocene strata in South Carolina based on dinoflagellate cysts (dinocysts), calcareous nannofossils, and pollen had been completed. This made it difficult to subdivide and correlate the Paleocene section in a detailed way. The object of the present report is to provide data on occurrences of Paleocene dinocysts, calcareous nannofossils, and pollen in samples from 15 boreholes in the coastal plain of South Carolina (table 1).

\section{Acknowledgments}

The authors acknowledge the efforts of Donald G. Queen, Eugene F. Cobbs II, Eugene F. Cobbs III, Gerald E. Idler, and especially David C. Prowell, all of whom work or did work for the U.S. Geological Survey, and Karen Waters and Joe Gellici of the South Carolina Department of Natural Resources, during coring and logging of the cores whose paleontology is reported here. We also thank Colleen Durand, Nancy Durika, Ellen Seefelt, Thomas Sheehan, and Karlyn Westover for diligent processing of the fossil-bearing samples. The U.S. Department of Energy supported this investigation. 
Table 1. South Carolina boreholes for which Paleocene dinoflagellate cyst, calcareous nannofossil, and pollen occurrence data are presented in this report and (or) have been presented in previous U. S. Geological Survey open-file reports.

\begin{tabular}{|c|c|c|c|c|c|c|c|c|c|c|c|c|c|c|}
\hline \multirow{2}{*}{$\begin{array}{c}\text { USGS } \\
\text { Drillhole } \\
\text { Designation }\end{array}$} & \multirow[b]{2}{*}{ Drillhole Name } & \multirow[b]{2}{*}{ County } & \multirow[b]{2}{*}{ Year } & \multirow{2}{*}{$\begin{array}{l}\text { Degrees } \\
\text { North } \\
\text { Latitude }\end{array}$} & \multirow{2}{*}{$\begin{array}{l}\text { Degrees } \\
\text { West } \\
\text { Longitude }\end{array}$} & \multirow{2}{*}{$\begin{array}{c}\text { Measured } \\
\text { Elevation, } \\
\text { Feet }\end{array}$} & \multirow{2}{*}{$\begin{array}{l}\text { Total } \\
\text { Depth, } \\
\text { Feet }\end{array}$} & \multirow[b]{2}{*}{ Samples } & \multicolumn{3}{|c|}{$\begin{array}{l}\text { Occurrence Data Presented } \\
\text { In This Report }\end{array}$} & \multicolumn{3}{|c|}{$\begin{array}{l}\text { Occurrence Data Previously } \\
\text { Provided in } \\
\text { USGS Open-File Reports } \\
\end{array}$} \\
\hline & & & & & & & & & $\begin{array}{l}\text { Dino- } \\
\text { flagellate } \\
\text { Cysts }\end{array}$ & $\begin{array}{c}\text { Calcareous } \\
\text { Nanno- } \\
\text { fossils }\end{array}$ & Pollen & $\begin{array}{l}\text { Dino- } \\
\text { flagellate } \\
\text { Cysts }\end{array}$ & $\begin{array}{c}\text { Calcareous } \\
\text { Nanno- } \\
\text { fossils }\end{array}$ & Pollen \\
\hline ALL-348 & DNR/DOE C10A & Allendale & 1990 & 33.013 & 81.2303 & 281.6 & 1,734 & cores & $\mathrm{X}$ & $\mathrm{X}$ & & & & \\
\hline BFT-2055 & Hilton Head Water test & Beaufort & 1992 & 32.1129 & 80.4214 & 10.0 & 3,833 & cuttings & $\mathrm{X}$ & & & & & \\
\hline BRK-644 & USGS/DNR St. Stephen & Berkeley & 1998 & 33.2415 & 79.5604 & 75.0 & 1,826 & cores & $\mathrm{X}$ & $\bar{X}$ & $\mathrm{X}$ & & & \\
\hline BRN-335 & SRS P21TA & Barnwell & 1985 & 33.0846 & 81.3628 & 207.0 & 1,152 & cores & $\mathrm{X}$ & & $\mathrm{X}$ & & & \\
\hline CHN-800 & USGS Cannon Park & Charleston & 1994 & 32.4655 & 79.5641 & 10.0 & 1,012 & cores & & $\mathrm{X}$ & & $\mathrm{X}$ & $\mathrm{X}$ & $\mathrm{X}$ \\
\hline CHN-802 & USGS Moores Landing & Charleston & 1996 & 32.5627 & 79.3927 & 7.8 & 369 & cores & & $\mathrm{X}$ & & & & \\
\hline CHN-803 & USGS Santee Coastal Reserve & Charleston & 1996 & 33.0910 & 79.2130 & 9.9 & 545 & cores & & $\mathrm{X}$ & & $\mathrm{X}$ & $\mathrm{X}$ & $\mathrm{X}$ \\
\hline COL-364 & USGS/DNR Edisto Island & Colleton & 1999 & 32.3013 & 80.1746 & 9.9 & 977 & cores & $\mathrm{X}$ & $\mathrm{X}$ & $\mathrm{X}$ & & & \\
\hline DOR-37 & USGS Clubhouse Crossroads No. 1 & Dorchester & 1975 & 32.5317 & 80.2133 & 18.0 & 2,599 & cores & $\mathrm{X}$ & $\mathrm{X}$ & $\mathrm{X}$ & $\mathrm{X}$ & $\mathrm{X}$ & $\mathrm{X}$ \\
\hline DOR-208 & USGS Pregnall & Dorchester & 1982 & 33.0908 & 80.2814 & 85.0 & 346 & cores & & $\mathrm{X}$ & & $\mathrm{X}$ & $\mathrm{X}$ & \\
\hline DOR-211 & USGS St. George & Dorchester & 1982 & 33.0925 & 80.3118 & 78.0 & 2,067 & cores & $\mathrm{X}$ & $\mathrm{X}$ & & & & \\
\hline JAS-426 & DNR/DOE C15 & Jasper & 1996 & 32.3704 & 80.5945 & 63.5 & 2,900 & sidewall cores & & & & & $\mathrm{X}$ & \\
\hline LEX-844 & USGS/DNR Swansea & Lexington & 1997 & 33.4444 & 81.0628 & 367.0 & 548 & cores & $\mathrm{X}$ & & $\mathrm{X}$ & & & \\
\hline ORG-393 & USGS/DNR Clark School No. 1 & Orangeburg & 1997 & 33.3029 & 80.5154 & 253.0 & 1,138 & cores & $\bar{X}$ & & $\mathrm{X}$ & & & \\
\hline ORG-430 & USGS/DNR Clark School No. 2 & Orangeburg & 1999 & 33.3029 & 80.5154 & 253.0 & 330 & cores & $\mathrm{X}$ & & $\mathrm{X}$ & & & \\
\hline
\end{tabular}




\section{Fossil Assemblages}

The fossil dinocyst, calcareous nannofossil, and pollen assemblages are presented in figures 3 through 28. These data are from 13 coreholes, one borehole from which sidewall cores were obtained, and one borehole from which cuttings were recovered (table 1). The table shows that some fossil data from some of these holes previously have been presented in U. S. Geological Survey open-file reports by Edwards and others (1997, 1999, 2000), Self-Trail and Bybell (1997), and Bybell and others (1998), but a subset of these data is updated in the present report along with data not previously made public. The diagrams for calcareous nannofossil assemblages do not display sample numbers (other than the depths from which the samples were obtained) because no such numbers were assigned. The coreholes and boreholes are presented in alphabetical order according to the county in which they were drilled. Hence, charts presenting data from each borehole are adjacent in the report, thereby permitting easy comparison of age interpretions based on different fossil groups from each borehole.

\section{Summary}

Twenty-six diagrams in this report display the occurrences of fossil dinocyst, calcareous nannofossil, and pollen assemblages in Paleocene samples from 13 coreholes, one borehole from which sidewall cores were obtained, and one borehole from which cuttings were recovered, all in South Carolina.

\section{References Cited}

Reference, 2000, National water quality inventory-1998 Report, U.S. Environmental Protection Agency Report EPA-841-F-00-006, Washington D.C.

Bybell, L. M., Conlon, K. J., Edwards, L. E., Frederiksen, N. O., Gohn, G. S., and Self-Trail, J. M., 1998, Biostratigraphy and physical stratigraphy of the USGS-Cannon Park core (CHN-800), Charleston County, South Carolina: U. S. Geological Survey Open-File Report 98-246, 65 p.

Drugg, W.S., 1967, Palynology of the upper Moreno Formation (Late Cretaceous-Paleocene) Escarpado Canyon, California: Palaeontographica, Abt. B, v. 120, p. 1-71.

Edwards, L.E., 1980, Dinoflagellate biostratigraphy: A first look, in Reinhardt, Juergen, and Gibson, T.G., Upper Cretaceous and lower Tertiary geology of the Chattahoochee River Valley, western Georgia and eastern Alabama, in Frey, R.W., ed., Excursions in southeastern geology, v. 2: Geological Society of America, Annual Meeting, (93rd), Atlanta 1980, Field Trip Guidebooks, p. 424-427, pl. 9, fig. 16.

Edwards, L.E., 1989, Dinoflagellate cysts from the lower Tertiary formations, Haynesville cores, Richmond County, Virginia, in Mixon, R.B., ed., Geology and paleontology of the Haynesville cores--Northeastern Virginia Coastal Plain: U.S. Geological Survey Professional Paper 1489-C, p. C1-C12.

Edwards, L. E., Bybell, L. M., Gohn, G. S., and Frederiksen, N. O., 1997, Paleontology and physical stratigraphy of the USGS-Pregnall No. 1 core (DOR-208), Dorchester County, South Carolina: U. S. Geological Survey Open-File Report OF 97-145, 35 p.

Edwards, L. E., Gohn, G. S., Self-Trail, J. M., Prowell, D. C., Bybell, L. M., Bardot, L. P., Firth, J. V., Huber, B. T., Frederiksen, N. O., and MacLeod, K. G., 1999, Physical stratigraphy, paleontology, and magnetostratigraphy of the USGS-Santee Coastal Reserve core (CHN-803), Charleston County, South Carolina: U. S. Geological Survey Open-File Report 99-308-A, 36 pp. 
Edwards, L. E., Gohn, G. S., Bybell, L. M., Chirico, P. G., Christopher, R. A., Frederiksen, N. O., Prowell, D. C., Self-Trail, J. M., and Weems, R. E., 2000, Supplement to the preliminary stratigraphic database for subsurface sediments of Dorchester County, South Carolina: U.S. Geological Survey Open-File Report 00-49-B.

Edwards, L.E., Goodman, D.K., and Witmer, R.J., 1984, Lower Tertiary (Pamunkey Group) dinoflagellate biostratigraphy, Potomac River area, Virginia and Maryland, in Frederiksen, N.O., and Krafft, K. (eds.), 1984, Cretaceous and Tertiary stratigraphy, paleontology, and structure, southwestern Maryland and northeastern Virginia: American Association of Stratigraphic Palynologists Field Trip Volume and Guidebook, p. 137-152.

Fallaw, W.C., and Price, V., 1995, Stratigraphy of the Savannah River Site and vicinity: Southeastern Geology, v. 35, p. 21-58.

Falls, W. F., and Prowell, D. C., 2001, Stratigraphy and depositional environments of sediments from five cores from Screven and Burke Counties, Georgia: U.S. Geological Survey Professional Paper 1603, p. A1-A20.

Hargrove, D. C., and Engelhardt, D. W., 1997, Palynology of the Maastrichtian/Danian boundary at Savannah River Site, South Carolina: Sedimentary Geology, v. 108, p. 121-140.

Kurita, Hiroshi, and McIntyre, D.J., 1995, Paleocene dinoflagellates from the Turtle Mountain Formation, southwestern Manitoba, Candada: Palynology, v. 19, p. 119-136, pls. 1, 2.

Morgenroth, Peter, 1966, Mikrofossilien uns Konkretionen des nordwesteuropäischen Untereozäns: Palaeontographica, ser. B, v. 119, p. 1-53, pls. 1-11.

Prowell, D.C., Edwards, L.E., and Frederiksen, N.O., 1985 [1986], The Ellenton Formation in South Carolina-A revised age designation from Cretaceous to Paleocene, in Stratigraphic notes, 1984: U.S. Geological Survey Bulletin 1605-A, p. A63-A69.

Self-Trail, J. M., and Bybell, L. M., 1997, Calcareous nannofossil biostratigraphy of the SCDNR test hole C-15, Jasper County, South Carolina: U. S. Geological Survey Open-File Report $97-$ 155,2 sheets.

Willoughby, R. H., Nystrom, P. G., Jr., Campbell, L. D., and Katuna, M. P., 1999, Cenozoic stratigraphic column of the coastal plain of South Carolina: South Carolina Geological Survey General Geologic Chart 1, 1 sheet. 


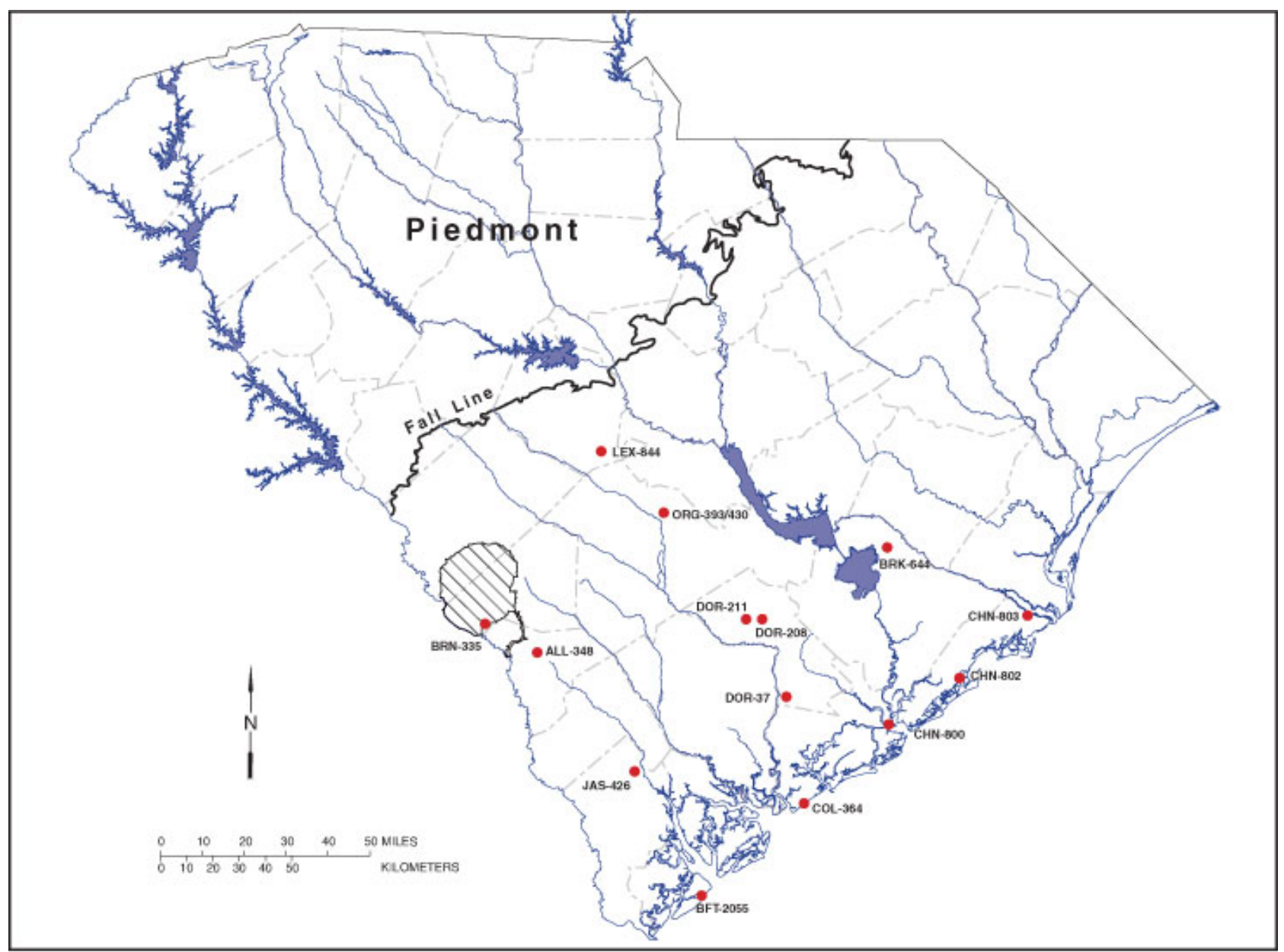

Figure 1. Index map of South Carolina showing the locations (black circles) and names of boreholes from which conventional cores, sidewall cores, or cuttings were recovered that were studied for this report, and the location of the Savannah River Site (hatched area). [file Norm Map-2.ai] 


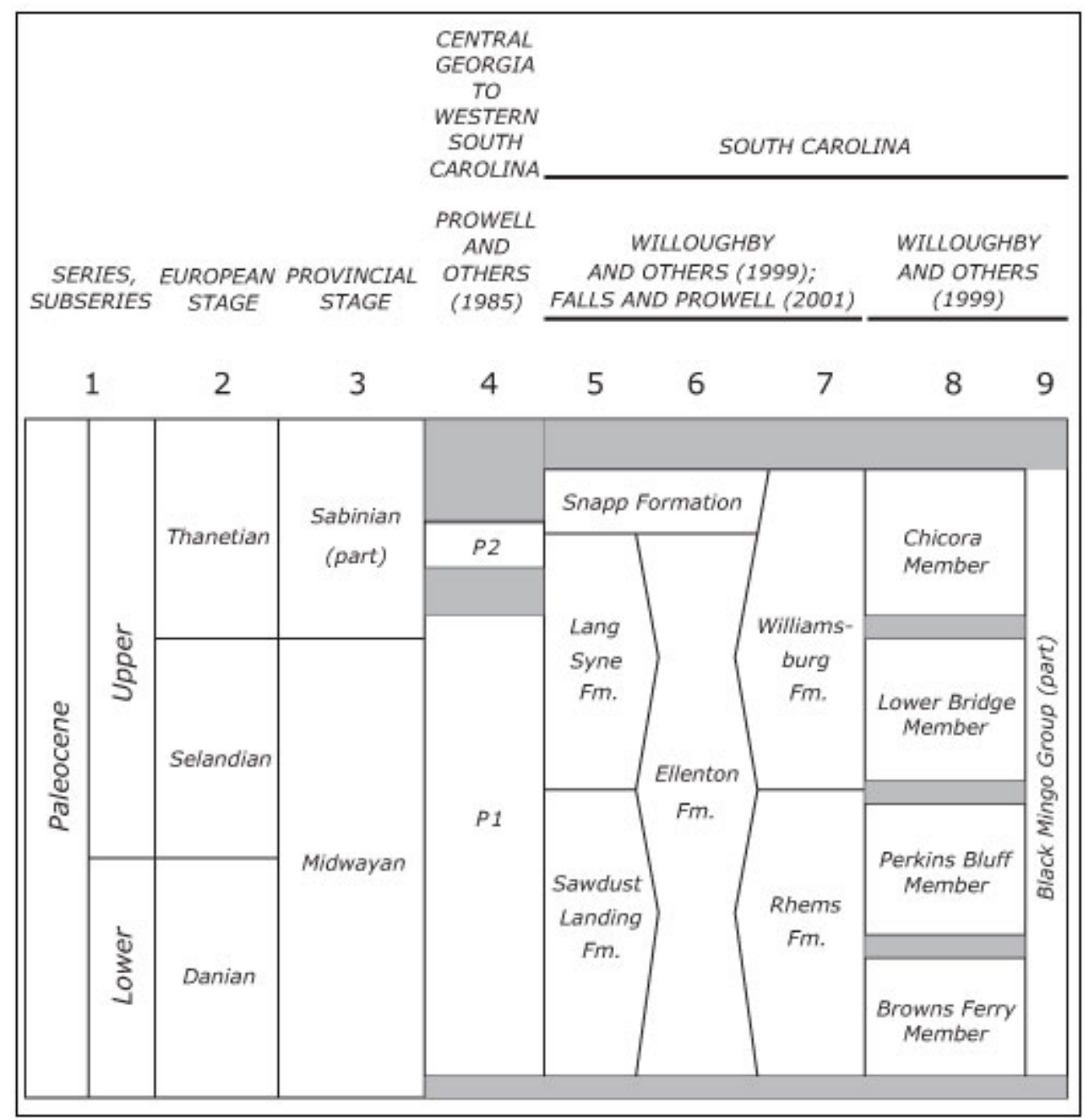

Figure 2. Generalized comparison of nomenclature used by several authors for Paleocene geologic units in South Carolina. Shaded areas indicate missing stratigraphic sections. Fm., formation. Columns 4-7 are slightly modified from Falls and Prowell (2001); in column 5, formations are updip units; in column 7, formations are downdip units. [file SE Paleocene fms-o file.ai] 


\begin{tabular}{|c|c|c|c|c|}
\hline Depth (feet) & 639.5 & 617 & 609 & 583.5 \\
\hline Sample & R4787 A & R4787 B & R4787 C & R4787 D \\
\hline Series & Paleocene & Barren & Barren & Eocene \\
\hline Part of Series & Upper & & & Lowest \\
\hline Taxa & & & & \\
\hline Adnatosphaeridium multispinosum Williams \& Downie 1966 & & & & $\mathrm{X}$ \\
\hline Adnatosphaeridium Williams \& Downie 1966 n. sp. & $\mathrm{X}$ & & & . \\
\hline Amphorosphaeridium multispinosum (Davey \& Williams 1966) Sarjeant 19 & $\mathrm{X}$ & & & $\mathrm{X}$ \\
\hline Apectodinium augustum/A. hyperacanthum & . & & & $\mathrm{X}$ \\
\hline Apectodinium homomorphum (Defl. \& Cook. 1955) Lentin \& Williams 197 & . & & & $\mathrm{X}$ \\
\hline Apectodinium paniculatum (Costa \& Downie 1976) Lentin \& Williams 197 & . & & & $\mathrm{X}$ \\
\hline Cerodinium medcalfi (Stover1974) Lentin \& Williams 1987 & . & & & $?$ \\
\hline Cerodinium speciosum (Alberti 1959) Lentin \& Williams 1987 & $\mathrm{X}$ & & & . \\
\hline Cordosphaeridium fibrospinosum Davey \& Williams 1966 & $\mathrm{X}$ & & & $\mathrm{X}$ \\
\hline Cordosphaeridium gracile (Eisenack 1954) Davey \& Williams 1966 & . & & & $\mathrm{X}$ \\
\hline Damassadinium californicum (Drugg 1967) Fensome et al. 1993 & $\mathrm{X}$ & & & . \\
\hline Dinopterygium cladoides sensu Morgenroth (1966) & . & & & $\mathrm{X}$ \\
\hline Eocladopyxis peniculata Morgenroth 1966 & . & & & $\mathrm{X}$ \\
\hline Fibrocysta bipolaris (Cookson \& Eisenack 1965) Stover \& Evitt 1978 & . & & & $\mathrm{X}$ \\
\hline Hafniasphaera septata (Cookson \& Eisenack 1967) Hansen 1977 & . & & & $\mathrm{X}$ \\
\hline Hystrichokolpoma unispinum Williams \& Downie 1966 & . & & & $\mathrm{X}$ \\
\hline Hystrichosphaeridium tubiferum (Erhenberg 1838) Deflandre 1937 & $\mathrm{X}$ & & & . \\
\hline Kallosphaeridium brevibarbatum de Coninck 1969 & 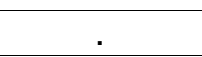 & & & $\mathrm{X}$ \\
\hline Nematosphaeropsis Deflandre \& Cookson 1955 n. sp. & $\mathrm{X}$ & & & . \\
\hline Operculodinium Wall $1967 \mathrm{sp.}$ & $\mathrm{X}$ & & & $\mathrm{X}$ \\
\hline Palaeocystodinium golzowense Alberti 1961 & $\mathrm{X}$ & & & . \\
\hline Phelodinium sp. of Edwards (1989) & $\mathrm{X}$ & & & . \\
\hline Polysphaeridium zoharyi (Rossignol 1962) Bujak et al. 1980 & & & & $?$ \\
\hline Senegalinium microgranulatum (Stanley 1965) Stover \& Evitt 1978 & $\mathrm{X}$ & & & . \\
\hline Spiniferites Mantell 1850 spp. & $X$ & & & $\mathrm{X}$ \\
\hline miscellaneous areoligeracean forms & . & & & $\mathrm{X}$ \\
\hline small peridiniacean forms & & & & $\mathrm{X}$ \\
\hline
\end{tabular}




\begin{tabular}{|c|c|c|c|}
\hline Depth & 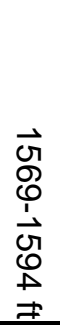 & 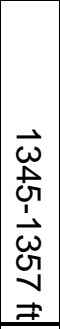 & 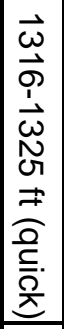 \\
\hline Sample & 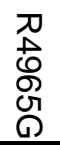 & $\begin{array}{l}D 0 \\
\stackrel{0}{0} \\
\text { ज़ } \\
\text { I }\end{array}$ & $\begin{array}{l}D \\
\mathbb{0} \\
\text { 品 } \\
\underline{\underline{G}}\end{array}$ \\
\hline Inferred Age & 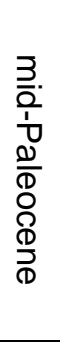 & 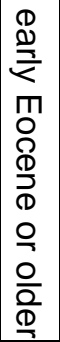 & 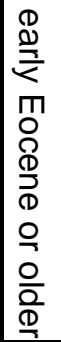 \\
\hline Taxa & & & \\
\hline Homotryblium plectilum Drugg \& Loeblich 1967 & $X$ & $\mathrm{X}$ & . \\
\hline Wetzeliella group & $\mathrm{X}$ & $\mathrm{X}$ & $\mathrm{x}$ \\
\hline Thalassiphora pelagica (Eisenack 1954) Eisenack \& Gocht 1960 & $\mathrm{X}$ & $\mathrm{X}$ & $\mathrm{X}$ \\
\hline Diphyes colligerum (Deflandre \& Cookson 1955) Cookson 1965 & . & $\mathrm{X}$ & . \\
\hline Pentadinium goniferum Edwards 1982 & $?$ & $\mathrm{X}$ & $\mathrm{X}$ \\
\hline Areosphaeridium diktyoplokus (Klumpp 1953) Eaton 1971 & . & $\mathrm{X}$ & . \\
\hline Rhombodinium draco Gocht 1955 & . & $\mathrm{X}$ & $\mathrm{x}$ \\
\hline Fibrocysta radiata (Morgenroth 1966) Stover \& Evitt 1978 & . & . & $\mathrm{x}$ \\
\hline Phelodinium sp. of Edwards (1989) & $\mathrm{x}$ & . & . \\
\hline Amphorosphaeridium multispinosum (Davey \& Williams 1966) Sarjeant & $\mathrm{x}$ & . & . \\
\hline Palaeoperidinium pyrophorum (Ehrenberg 1838) Sarjeant 1967 & $\mathrm{x}$ & . & . \\
\hline Damassadinium californicum (Drugg 1967) Fensome et al. 1993 & $\mathrm{x}$ & . & . \\
\hline Deflandrea delineata & $\mathrm{x}$ & . & . \\
\hline Senegalinium micrograulatum (Stanley 1965) Stover \& Evitt 1978 & $\mathrm{x}$ & . & . \\
\hline \multicolumn{4}{|l|}{ Figure 4. Distribution of dinocyst taxa in Paleocene cuttings samples from the } \\
\hline Hilton Head water test borehole (BFT-2055), Beaufort County, South Carolina. & & & \\
\hline
\end{tabular}




\begin{tabular}{|c|c|c|c|c|c|c|c|c|c|c|c|c|c|c|c|c|c|}
\hline & Depth $(\mathrm{Ft})$ & 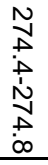 & 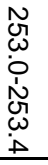 & $\begin{array}{l}\tilde{N} \\
\omega \\
\dot{\omega} \\
\dot{\Lambda} \\
\omega \\
\omega \\
\Delta\end{array}$ & 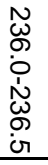 & $\begin{array}{l}\tilde{\omega} \\
\omega \\
\stackrel{N}{+} \\
\stackrel{\sim}{\omega} \\
\omega \\
\omega \\
\infty \\
\infty\end{array}$ & $\begin{array}{l}N \\
N \\
o \\
\text { N } \\
\tilde{N} \\
N \\
\infty \\
\infty\end{array}$ & 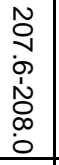 & 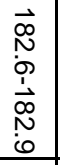 & 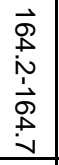 & 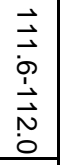 & $\begin{array}{l}\varnothing \\
0 \\
i \\
\dot{0} \\
i \\
\text { in }\end{array}$ & $\begin{array}{l}\infty \\
\stackrel{\infty}{\sim} \\
\stackrel{0}{0} \\
\stackrel{0}{0} \\
0\end{array}$ & 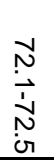 & $\begin{array}{l}8 \\
\circ \\
0 \\
\dot{1} \\
\circ \\
\dot{1} \\
\end{array}$ & 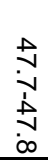 & 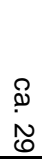 \\
\hline & Sample & $\begin{array}{l}\text { 刃ु } \\
\text { О } \\
\text { ه } \\
\text { m }\end{array}$ & $\begin{array}{l}\text { 刃ु } \\
\text { Д̆ } \\
8 \\
\pi\end{array}$ & 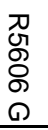 & $\begin{array}{l}\text { ग } \\
\text { Оे } \\
\varnothing \\
\text { エ }\end{array}$ & $\begin{array}{l}\text { 刃ु } \\
\text { ळ } \\
\text { ᄋ } \\
\end{array}$ & \begin{tabular}{l} 
गु \\
Oे \\
8 \\
\hdashline
\end{tabular} & 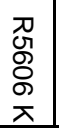 & $\begin{array}{l}\text { गु } \\
\text { Oे } \\
\stackrel{\circ}{\circ} \\
\end{array}$ & $\begin{array}{l}\text { D } \\
\text { Oे } \\
8 \\
3 \\
3\end{array}$ & $\begin{array}{l}\text { गु } \\
\text { Oे } \\
8 \\
0\end{array}$ & $\begin{array}{l}\text { गु } \\
\text { Oे } \\
8 \\
0\end{array}$ & $\begin{array}{l}\text { 刃 } \\
\text { Oু } \\
8 \\
8 \\
0 \\
\end{array}$ & $\begin{array}{l}\text { गु } \\
\text { ळे } \\
8 \\
0\end{array}$ & $\begin{array}{l}\text { 刃ु } \\
\text { ठ } \\
\text { 心 }\end{array}$ & 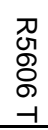 & $\begin{array}{l}\text { 刃ु } \\
\text { ळ } \\
\varnothing \\
\subset\end{array}$ \\
\hline & Series & \multicolumn{13}{|c|}{ Paleocene } & \multicolumn{3}{|c|}{ Pal-Eoc.? } \\
\hline & Subseries & \multicolumn{9}{|c|}{ Lower } & \multicolumn{4}{|c|}{ Upper } & & & \\
\hline Taxa & & & & & & & & & & & & & & & & & \\
\hline Achilleodinium biformoides (Eisenack 1954) Eaton 1976 & & . & . & . & . & . & . & . & . & . & . & . & . & & . & . & $x$ \\
\hline chorate, cf. Achilleodinium & & . & . & . & . & . & . & . & . & . & . & . & . & . & $\mathrm{X}$ & $\mathrm{x}$ & . \\
\hline Achomosphaera alcicornu (Eisenack 1954) Davey \& Williams 1966 & & $\mathrm{X}$ & . & . & . & . & . & . & . & . & . & $\mathrm{x}$ & $\mathrm{x}$ & . & . & . & . \\
\hline Adnatosphaeridium multispinosum Williams \& Downie 1966 & & . & . & . & . & . & . & . & . & . & . & . & $\mathrm{x}$ & $\mathrm{x}$ & $\mathrm{x}$ & $\mathrm{x}$ & $\mathrm{x}$ \\
\hline Amphorosphaeridium multispinosum (Davey \& Williams 1966) Sarjeant 1981 & & . & . & . & . & . & . & . & . & . & . & . & . & $\mathrm{X}$ & . & . & . \\
\hline Andalusiella polymorpha (Malloy 1972) Lentin \& Williams 1977 & & . & . & . & . & . & . & . & $\mathrm{x}$ & $\mathrm{x}$ & . & . & . & & . & . & . \\
\hline Andalusiella sp. aff. A. polymorpha of Edwards (1980) & & . & $\mathrm{X}$ & $?$ & . & . & . & . & . & . & . & . & . & . & . & . & . \\
\hline ?Andalusiella rhombohedra of Edwards and others (1984) & & . & $\mathrm{x}$ & . & . & . & . & . & $\mathrm{X}$ & . & . & . & . & . & . & . & . \\
\hline Apectodinium homomorphum (Deflandre \& Cookson 1955) Lentin \& Williams 1977 & &. & . & . & . & . & . & . & . & . & . & . & $\mathrm{x}$ & $\mathrm{x}$ & $\mathrm{x}$ & $\mathrm{x}$ & . \\
\hline Apectodinium hyperacanthum (Deflandre \& Cookson 1955) Lentin \& Williams 1977 & &. & & & . & & . & . & & . & . & . & . & & $\mathrm{x}$ & & \\
\hline Carpatella cornuta Grigorovich 1969 & & $?$ & & $?$ & . & $\mathrm{X}$ & . & . & & $\mathrm{X}$ & . & . & . & & . & . & . \\
\hline Catillopsis Drugg $1970 ?$ sp. & & . & $\mathrm{x}$ & . & . & $\mathrm{x}$ & . & . & . & . & . & . & . & . & . & . & . \\
\hline Cerodinium diebelii (Alberti 1959) Lentin \& Williams 1987 & & . & . & $\mathrm{x}$ & . & . & $\mathrm{x}$ & . & . & . & . & . & . & . & . & . & . \\
\hline Cerodinium striatum (Drugg 1967) Lentin \& Williams 1987 & & $\mathrm{x}$ & $\mathrm{x}$ & . & . & . & . & . & . & . & . & . & . & . & . & . & . \\
\hline Cerodinium Vozzhennikova $1963 \mathrm{sp.}$ & & . & . & . & . & $\mathrm{x}$ & . & . & . & . & . & . & . & . & . & . & . \\
\hline Cordosphaeridium fibrospinosum Davey \& Williams 1966 & & $\mathrm{x}$ & $\mathrm{x}$ & . & . & & . & $\mathrm{x}$ & $\mathrm{x}$ & . & . & . & . & & . & . & $\mathrm{x}$ \\
\hline Cordosphaeridium gracile (Eisenack 1954) Davey \& Williams 1966 & & . & . & . & . & . & . & . & & . & . & . & . & $\mathrm{x}$ & $\mathrm{x}$ & $\mathrm{x}$ & $\mathrm{x}$ \\
\hline Cordosphaeridium inodes (Klumpp 1953) Eisenack 1963 & & . & . & $\mathrm{x}$ & . & . & $\mathrm{x}$ & . & $\mathrm{x}$ & . & . & . & . & . & . & . & . \\
\hline Cordosphaeridium Eisenack 1963 sp. & & . & . & . & . & $\mathrm{X}$ & . & $\mathrm{x}$ & . & . & $\mathrm{X}$ & $\mathrm{x}$ & $\mathrm{x}$ & . & . & . & . \\
\hline Cribroperidinium Neale \& Sarjeant 1962 spp. & & $\mathrm{x}$ & $\mathrm{x}$ & $\mathrm{x}$ & . & $\mathrm{X}$ & $\mathrm{x}$ & . & . & . & . & . & . & . & . & . & . \\
\hline Damassadinium californicum (Drugg 1967) Fensome et al. 1993 & & $\mathrm{x}$ & $\mathrm{x}$ & $\mathrm{x}$ & $\mathrm{x}$ & $\mathrm{x}$ & $\mathrm{x}$ & $\mathrm{x}$ & . & $\mathrm{x}$ & $\mathrm{x}$ & $\mathrm{x}$ & $\mathrm{x}$ & $\mathrm{x}$ & $\mathrm{x}$ & $?$ & ?f \\
\hline Deflandrea cf. D. diebelii Alberti of Drugg (1967) & & . & $\mathrm{x}$ & . & . & 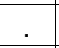 & . & $\mathrm{x}$ & & $\mathrm{x}$ & . & . & . & & . & . & 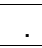 \\
\hline Deflandrea n. sp. aff. D. truncata Eisenack 1938 & & . & $\mathrm{x}$ & $\mathrm{x}$ & $\mathrm{x}$ & & . & $\mathrm{x}$ & & $\mathrm{x}$ & . & & . & & . & . & \\
\hline Deflandrea phosphoritica Eisenack 1938 & & . & . & . & . & . & . & . & . & . & . & . & $\mathrm{x}$ & $?$ & $?$ & . & $\mathrm{x}$ \\
\hline Diphyes colligerum (Deflandre \& Cookson 1955) Cookson 1965 & & $\mathrm{x}$ & $\mathrm{x}$ & $\mathrm{x}$ & . & $\mathrm{P}$ & $\mathrm{P}$ & . & $\mathrm{x}$ & . & $\mathrm{x}$ & . & . & . & . & . & . \\
\hline Disphaerogena carposphaeropsis Wetzel 1933 & & $\mathrm{x}$ & $\mathrm{x}$ & . & . & . & . & . & . & . & . & . & . & . & . & . & . \\
\hline Eocladopyxis peniculata Morgenroth 1966 & & . & . & . & . & . & . & . & . & . & . & $\mathrm{x}$ & $\mathrm{x}$ & $\mathrm{x}$ & $\mathrm{x}$ & $\mathrm{x}$ & $\mathrm{x}$ \\
\hline Exochosphaeridium Davey et al. 1966 spp. & & . & $\mathrm{x}$ & $\mathrm{x}$ & $\mathrm{x}$ & $\mathrm{x}$ & $\mathrm{x}$ & $\mathrm{x}$ & $\mathrm{x}$ & $\mathrm{x}$ & $\mathrm{x}$ & & . & & . & . & \\
\hline Fibradinium annetorpense Morgenroth 1968 & & $\mathrm{X}$ & $\mathrm{x}$ & . & . & $?$ & . & . & . & . & . & . & . & . & . & . & . \\
\hline Fibrocysta radiata (Morgenroth 1966) Stover \& Evitt 1978 & & . & . & . & . & . & . & . & . & . & . & $\mathrm{x}$ & $\mathrm{x}$ & $\mathrm{x}$ & . & . & . \\
\hline Fibrocysta Stover \& Evitt 1978 sp. & & . & . & . & . & $\mathrm{x}$ & . & . & & . & & & . & & . & & \\
\hline
\end{tabular}




\begin{tabular}{|c|c|c|c|c|c|c|c|c|c|c|c|c|c|c|c|c|c|}
\hline & Depth $(\mathrm{Ft})$ & 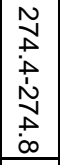 & $\begin{array}{l}N \\
\tilde{N} \\
\omega \\
0 \\
\dot{N} \\
\tilde{W} \\
0 \\
D\end{array}$ & 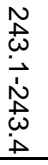 & $\begin{array}{l}\tilde{\omega} \\
o \\
0 \\
\dot{1} \\
\tilde{\omega} \\
\tilde{\omega} \\
\dot{\omega}\end{array}$ & $\begin{array}{l}\tilde{\omega} \\
\omega \\
\dot{\omega} \\
\dot{1} \\
\tilde{\omega} \\
\omega \\
\infty \\
\infty\end{array}$ & $\begin{array}{l}N \\
N \\
o \\
01 \\
N \\
N \\
N \\
\infty\end{array}$ & $\begin{array}{l}\tilde{0} \\
\text { V } \\
0 \\
1 \\
\tilde{O} \\
0 \\
0 \\
0\end{array}$ & 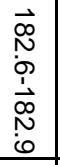 & 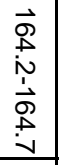 & 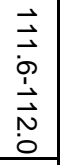 & $\begin{array}{l}0 \\
0 \\
i \\
0 \\
\text { ì } \\
\text { N }\end{array}$ & $\begin{array}{c}\infty \\
\stackrel{\infty}{N} \\
\stackrel{0}{0} \\
\stackrel{0}{0} \\
\end{array}$ & $\begin{array}{l}N \\
N \\
i \\
N \\
N \\
G\end{array}$ & $\begin{array}{l}8 \\
0 \\
0 \\
\dot{2} \\
\dot{1} \\
\end{array}$ & \begin{tabular}{l} 
\pm \\
\multirow{2}{n}{} \\
$\dot{1}$ \\
$\infty$ \\
0
\end{tabular} & $\begin{array}{l}\text { مै } \\
\text { Nֶ }\end{array}$ \\
\hline & Sample & $\begin{array}{l}\text { Dू } \\
\text { Oू } \\
8 \\
m \\
\end{array}$ & $\begin{array}{l}\text { 刃 } \\
\text { Л } \\
8 \\
\pi\end{array}$ & 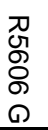 & $\begin{array}{l}\text { गू } \\
\text { O } \\
\text { o } \\
\text { I }\end{array}$ & $\begin{array}{l}\text { 刃ू } \\
\text { Оి } \\
0 \\
\end{array}$ & $\begin{array}{l}\text { गू } \\
\text { ठे } \\
\varnothing \\
\end{array}$ & $\begin{array}{l}\text { 刃 } \\
\text { Oे } \\
\text { O } \\
\pi\end{array}$ & $\begin{array}{l}\text { गु } \\
\text { Oे } \\
\varnothing \\
\end{array}$ & $\begin{array}{l}\text { गू } \\
\text { Oे } \\
8 \\
3\end{array}$ & $\begin{array}{l}\text { ㄲ. } \\
\text { O } \\
8 \\
0\end{array}$ & $\begin{array}{l}\text { ग } \\
\text { Oे } \\
8 \\
0\end{array}$ & $\begin{array}{l}\text { 刃 } \\
\text { Oू } \\
8 \\
0 \\
0\end{array}$ & $\begin{array}{l}\prod_{\pi} \\
\text { Oे } \\
0 \\
0\end{array}$ & $\begin{array}{l}\text { ग } \\
\text { Oे } \\
\text { o } \\
\text { 心 }\end{array}$ & $\begin{array}{l}\text { गु } \\
\text { ᄋे } \\
8 \\
-1\end{array}$ & $\begin{array}{l}\text { गु } \\
\text { ᄋ } \\
\text { ه } \\
\subset\end{array}$ \\
\hline & Series & \multicolumn{13}{|c|}{ Paleocene } & \multicolumn{3}{|c|}{ Pal-Eoc.? } \\
\hline & Subseries & \multicolumn{9}{|c|}{ Lower } & \multicolumn{4}{|c|}{ Upper } & & & \\
\hline Taxa & & & & & & & & & & & & & & & & & \\
\hline Fromea fragilis (Cookson \& Eisenack 1962) Stover \& Evitt 1978 & & $\mathrm{x}$ & & . & . & . & . & & . & . & $\mathrm{x}$ & . & . & . & . & . & . \\
\hline Hafniasphaera fluens Hansen 1977 & & $\mathrm{x}$ & $\mathrm{x}$ & $\mathrm{X}$ & . & $\mathrm{X}$ & . & $\mathrm{x}$ & . & . & . & . & . & . & . & . & . \\
\hline Hafniasphaera septata (Cookson \& Eisenack 1967) Hansen 1977 & & . & . & . & . & $\mathrm{x}$ & . & . & . & . & . & $\mathrm{X}$ & . & . & . & . & . \\
\hline Hafniasphaera Hansen 1977 sp. & & . & . & . & . & . & . & . & $\mathrm{x}$ & . & . & . & $\mathrm{x}$ & . & . & . & . \\
\hline Hystrichokolpoma Deflandre 1935 sp. & & $\mathrm{x}$ & $\mathrm{x}$ & $\mathrm{X}$ & . & $\mathrm{x}$ & $\mathrm{X}$ & & . & . & . & $\mathrm{x}$ & $\mathrm{X}$ & $\mathrm{X}$ & $\mathrm{X}$ & $\mathrm{x}$ & . \\
\hline Ifecysta Jan du Chêne \& Adediran 1985 sp. & & $\mathrm{x}$ & $\mathrm{x}$ & $\mathrm{x}$ & . & . & . & . & . & $\mathrm{x}$ & . & . & & . & . & . & . \\
\hline Isabelidinium/Chatangiella/Manumiella sp. (reworked?) & & . & . & . & . & . & . & . & . & . & . & . & . & . & $\mathrm{X}$ & . & . \\
\hline Kallosphaeridium brevibarbatum de Coninck 1969 & & . & . & . & . & . & . & . & . & . & . & $\mathrm{X}$ & $\mathrm{x}$ & $?$ & $?$ & . & . \\
\hline Lejeunecysta Artzner \& Dörhöfer 1978 sp. & & . & $\mathrm{x}$ & & & . & . & . & . & . & . & $\mathrm{x}$ & . & . & $\mathrm{x}$ & . & . \\
\hline Lingulodinium machaerophorum (Deflandre \& Cookson 1955) Wall 1967 & & . & & $\therefore$ & & . & . & & . & & . & . & & . & $\mathrm{x}$ & . & . \\
\hline Melitasphaeridium pseudorecurvatum (Morgenroth 1966) Bujak et al. 1980 & & . & & . & & . & . & & . & . & . & . & . & $\mathrm{x}$ & . & . & . \\
\hline Membranosphaera maastrichtica Samoilovitch 1961 & & . & $?$ & $\mathrm{x}$ & . & . & . & . & . & . & . & . & . & . & . & . & . \\
\hline Oligosphaeridium complex (White 1842) Davey \& Williams 1966 & & $?$ & . & . & . & . & $?$ & . & . & . & . & . & . & . & . & . & . \\
\hline Operculodinium Wall 1967 sp. or spp. & & $\mathrm{X}$ & . & . & $\mathrm{x}$ & $\mathrm{X}$ & $\mathrm{X}$ & . & $\mathrm{x}$ & $\mathrm{x}$ & $\mathrm{x}$ & . & . & $\mathrm{x}$ & $\mathrm{X}$ & $\mathrm{x}$ & . \\
\hline Palaeocystodinium golzowense Alberti 1961 & & . & . & . & . & . & . & . & . & $?$ & $\mathrm{x}$ & . & . & . & . & . & . \\
\hline Palaeocystodinium Alberti $1961 \mathrm{sp}$. & & $\mathrm{x}$ & $\mathrm{x}$ & . & . & . & . & $\mathrm{x}$ & . & $\mathrm{x}$ & $\mathrm{x}$ & . & & . & . & . & . \\
\hline Palaeocystodinium Alberti 1961 sp. (fat) & & . & & . & . & $\mathrm{x}$ & $\mathrm{x}$ & $\mathrm{x}$ & $\mathrm{x}$ & . & . & . & . & . & . & . & . \\
\hline Palaeoperidinium pyrophorum (Ehrenberg 1838) Sarjeant 1967 & & . & $\mathrm{x}$ & $\mathrm{x}$ & . & . & . & $\mathrm{x}$ & . & . & $\mathrm{x}$ & . & . & . & . & . & . \\
\hline Phelodinium magnificum (Stanley 1965) Stover \& Evitt 1978 & & $\mathrm{X}$ & . & . & . & . & . & . & . & . & . & . & . & . & . & . & . \\
\hline Phelodinium sp. of Edwards (1989) & & . & . & . & . & . & . & . & . & . & $\mathrm{x}$ & . & . & poor & . & . & . \\
\hline Phelodinium Stover \& Evitt 1978 spp. & & . & $\mathrm{x}$ & $\mathrm{x}$ & $\mathrm{x}$ & $\mathrm{x}$ & $\mathrm{x}$ & & $\mathrm{x}$ & $\mathrm{x}$ & . & . & . &. & . & . & . \\
\hline Pulchrasphaera minuscula Schiøler et al. 1997 & & $\mathrm{x}$ & & . & $?$ & . & $?$ & & . & . & . & . & . & . & . & . & . \\
\hline Rottnestia Cookson \& Eisenack 1961 sp. & & . & & . & . & . & . & $\mathrm{x}$ & . & . & . & . & . & . & . & . & . \\
\hline Senoniasphaera Clarke \& Verdier 1967 sp. & & . & . & . & . & . & . & . & $?$ & $?$ & . & . & . & . & . & . & . \\
\hline Spinidinium pulchrum (Benson 1976) Lentin \& Williams 1977 & & $?$ & . & $?$ & . & . & . & . & $?$ & $?$ & . & . & . & . & . & . & . \\
\hline Spinidinium Cookson \& Eisenack 1962 spp. & & $\mathrm{x}$ & $\mathrm{x}$ & $\mathrm{x}$ & $\mathrm{x}$ & $\mathrm{x}$ & $\mathrm{x}$ & $\mathrm{x}$ & $\mathrm{x}$ & $\mathrm{x}$ & $\mathrm{x}$ & $\mathrm{x}$ & . & . & . & . & . \\
\hline Spiniferites pseudofurcatus (Klumpp 1953) Sarjeant 1970 & & . & . & . & . & . & . & . & $?$ & . & . & . & . & . & . & . & . \\
\hline Spiniferites Mantell 1850 spp. & & $\mathrm{x}$ & $\mathrm{x}$ & $\mathrm{x}$ & $\mathrm{x}$ & $\mathrm{x}$ & $\mathrm{x}$ & $\mathrm{x}$ & $\mathrm{x}$ & $\mathrm{x}$ & $\mathrm{X}$ & $\mathrm{x}$ & $\mathrm{X}$ & $\mathrm{x}$ & $\mathrm{x}$ & $\mathrm{x}$ & $\mathrm{x}$ \\
\hline Tectatodinium rugulatum (Hansen 1977) McMinn 1988 (sensu amplo) & & . & . & . & . & . & . & $\mathrm{X}$ & $\mathrm{x}$ & $\mathrm{x}$ & . & . & . & . & . & . & . \\
\hline Turbiosphaera sp. aff T. magnifica Eaton of Edwards (1989) (frag.) & & . & . & . & . & . & . & . & . & . & . & ?f & . & . & . & . & . \\
\hline Xenicodinium-Tectatodinium plexus & & $\mathrm{x}$ & & . & & . & $?$ & & . & & . & . & & & & . & \\
\hline
\end{tabular}




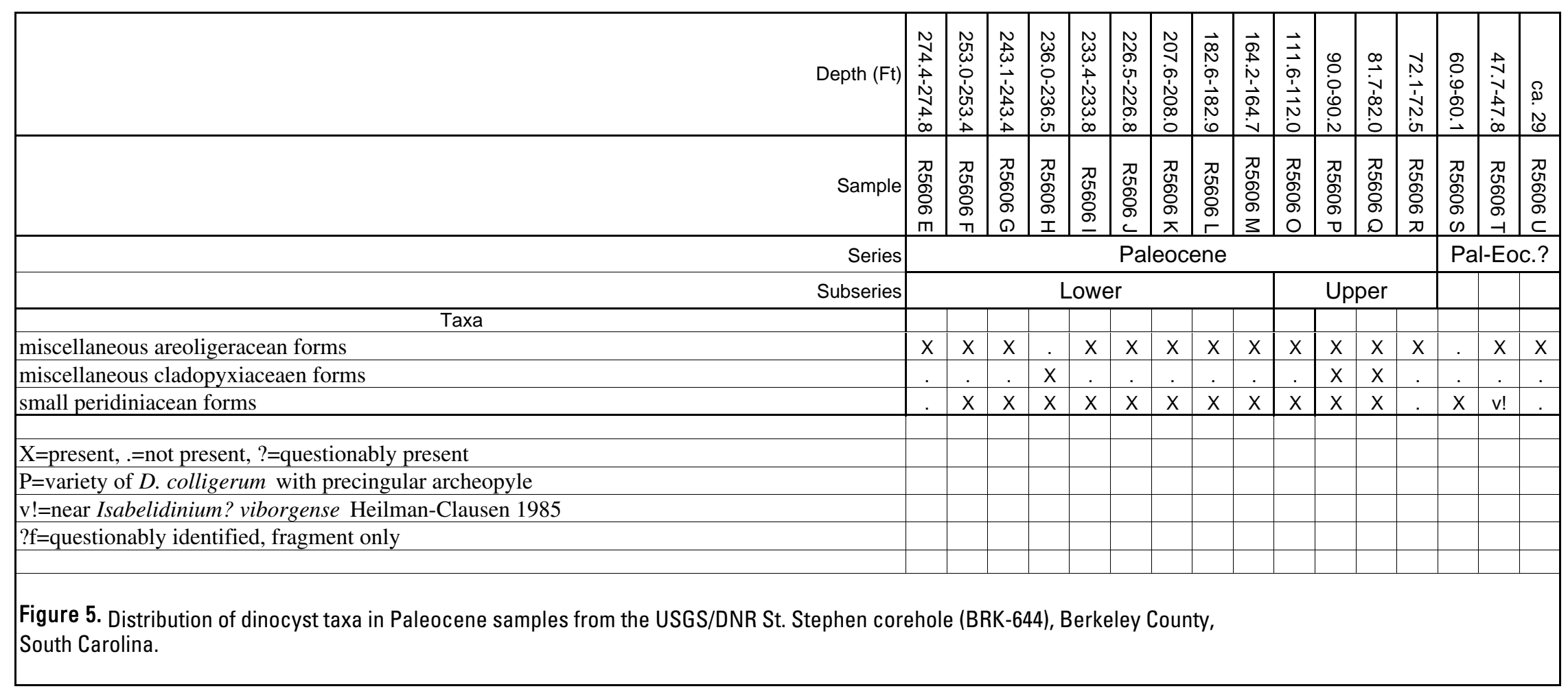




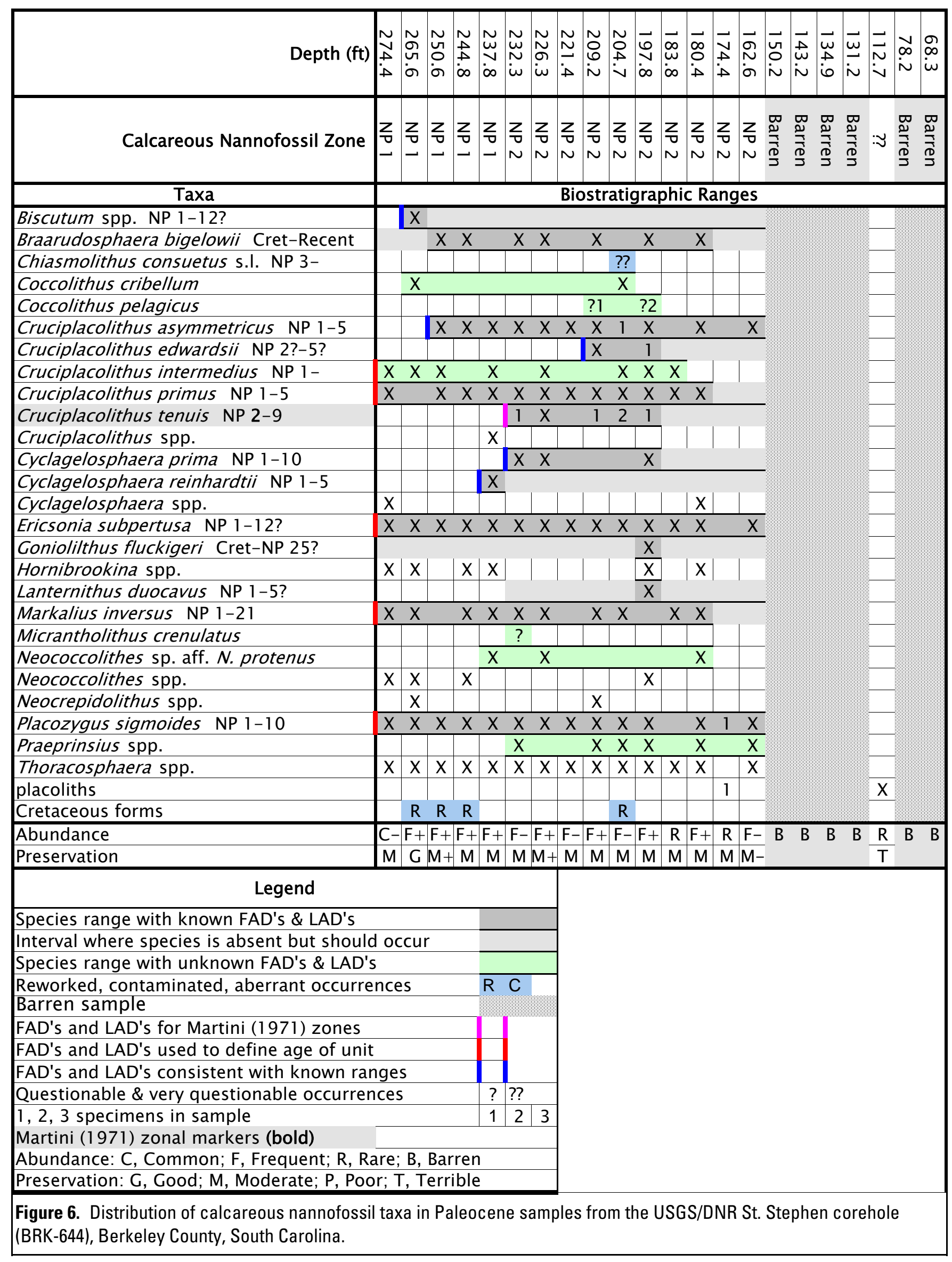




\begin{tabular}{|c|c|c|c|c|c|c|c|c|c|c|c|c|c|c|c|c|c|}
\hline Depth $(\mathrm{ft})$ & 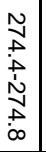 & \begin{tabular}{|c|}
$\tilde{N}$ \\
$\omega$ \\
0 \\
$\dot{1}$ \\
$\tilde{N}$ \\
$\omega$ \\
$\Delta$ \\
0
\end{tabular} & $\mid \begin{array}{c}\tilde{\omega} \\
\omega \\
\dot{1} \\
\tilde{\omega} \\
\omega \\
\dot{\Delta} \\
\end{array}$ & 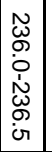 & \begin{tabular}{|c|}
$\tilde{w}$ \\
$\omega$ \\
$\omega$ \\
$\dot{1}$ \\
$\tilde{\omega}$ \\
$\omega$ \\
$\infty$ \\
$\infty$
\end{tabular} & 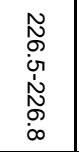 & 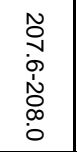 & $\begin{array}{c}\vec{c} \\
o \\
N \\
0 \\
i \\
\dot{1} \\
N \\
0 \\
0\end{array}$ & 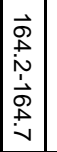 & $\begin{array}{l}\vec{\omega} \\
\stackrel{+}{+} \\
\dot{0} \\
\dot{\omega} \\
心 \\
\stackrel{\Delta}{\Delta}\end{array}$ & 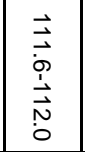 & $\begin{array}{l}8 \\
0 \\
\dot{1} \\
\dot{0} \\
\dot{N}\end{array}$ & 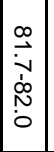 & $\begin{array}{l}\stackrel{N}{N} \\
\stackrel{i}{N} \\
\text { טn }\end{array}$ & $\begin{array}{l}8 \\
0 \\
0 \\
0 \\
0 \\
0\end{array}$ & \begin{tabular}{l} 
\pm \\
\multirow{1}{N}{} \\
$\dot{N}$ \\
$\infty$
\end{tabular} & $\begin{array}{l}\stackrel{\rho}{p} \\
\tilde{0}\end{array}$ \\
\hline Sample & $\begin{array}{l}\text { गु } \\
\text { Oे } \\
\text { m } \\
m\end{array}$ & 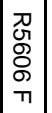 & 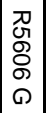 & 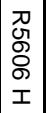 & $\begin{array}{l}0 \\
\widetilde{N} \\
\stackrel{0}{0} \\
-\end{array}$ & $\begin{array}{l}\text { 刃ु } \\
\text { ठ̀े } \\
\stackrel{2}{4}\end{array}$ & $\begin{array}{l}\text { 刃 } \\
\text { Oे } \\
\text { Ф } \\
\text { त }\end{array}$ & $\begin{array}{l}D_{0} \\
\text { Oे } \\
8 \\
\ulcorner\end{array}$ & 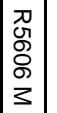 & $\begin{array}{l}\text { 刃ु } \\
\text { Oे } \\
\text { O } \\
z\end{array}$ & $\begin{array}{l}\text { 刃 } \\
\text { Оे } \\
8 \\
0\end{array}$ & $\begin{array}{l}\text { 刃 } \\
\text { Oे } \\
\text { ه } \\
0\end{array}$ & 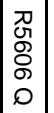 & $\begin{array}{l}\text { 刃ु } \\
\text { Oे } \\
\text { ه } \\
\text { D }\end{array}$ & $\begin{array}{l}\text { D } \\
\text { Oे } \\
\text { O } \\
\text { 心 }\end{array}$ & $\begin{array}{l}\text { 刃ु } \\
\text { Oे } \\
\stackrel{-}{-1} \\
-1\end{array}$ & $\begin{array}{l}\text { 刃ु } \\
\text { 。े } \\
\stackrel{\circ}{\subset} \\
\end{array}$ \\
\hline $\begin{array}{r}\text { Age or NP zone } \\
\text { equivalent }\end{array}$ & 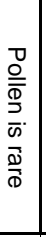 & 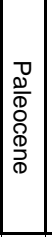 & 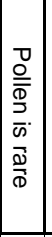 & 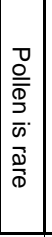 & 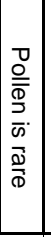 & 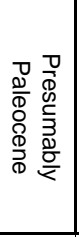 & 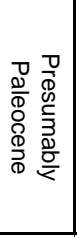 & 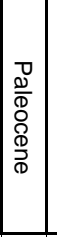 & 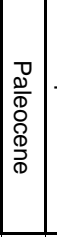 & 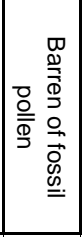 & 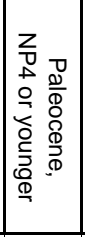 & 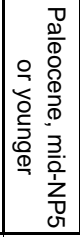 & $\begin{array}{l}z \\
0 \\
0\end{array}$ & zo & 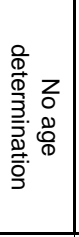 & 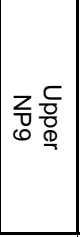 & 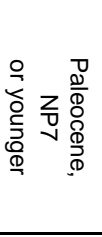 \\
\hline Taxa & . & . &. & . &. & . & . & . & . & . & . & . &. & . &. & . & . \\
\hline Aesculiidites circumstriatus & . & & . & . & . & & - & & & . & . & - & $\mathrm{X}$ & $\mathrm{x}$ & & $\mathrm{X}$ & . \\
\hline Bombacacides reticulatus & . & $\mathrm{X}$ &. & . & . & $\mathrm{X}$ & $\mathrm{X}$ & $\mathrm{X}$ & $\mathrm{X}$ & . & . & $\mathrm{X}$ & $\mathrm{X}$ & $\mathrm{X}$ & $\mathrm{X}$ & . & . \\
\hline Carya $<29 \mu \mathrm{m}$ & . & . &. &. &. & . & . & . &. & . & . & $\mathrm{X}$ & $\mathrm{X}$ & $\mathrm{X}$ & . & . & . \\
\hline Caryapollenites prodromus group & . & . &. &. &. & . & . & $x$ & & . & . & . & . &. & . & . & . \\
\hline Favitricolporites baculoferus & & &. &. & . & & 7 & & $\mathrm{x}$ & . & . & . & . & . & . & . & . \\
\hline Holkopollenites chemardensis & . & $\mathrm{X}$ & . & . & . & . & $\mathrm{X}$ & $\mathrm{X}$ & $\mathrm{X}$ & . & . & . & . & . & . & . & . \\
\hline Intratriporopollenites pseudinstructus & . & . & . & . & . & . & . &. &. & . & & . & . & $\mathrm{x}$ & . & $\mathrm{x}$ & . \\
\hline Momipites actinus & . & . & . &. & . & . & . & & & . & $\mathrm{x}$ & . &. & . & . & . & . \\
\hline Momipites coryloides/microfoveolatus & . & $\mathrm{X}$ & . &. &. & . & . & $\mathrm{X}$ & $\mathrm{X}$ & . & $\mathrm{X}$ & $\mathrm{X}$ & $\mathrm{x}$ & $\mathrm{X}$ & . & prob. & $?$ \\
\hline Momipites strictus & . & . &. &. &. & & . & . &. & . & & . & $\mathrm{x}$ & $\mathrm{X}$ & . &. & $\mathrm{x}$ \\
\hline Momipites tenuipolus group & & . & . &. &. & $\mathrm{X}$ & . & . & & . & $\mathrm{X}$ & . &. & . & . & . &. \\
\hline Nudopollis terminalis & . & . & . &. & . & . & . & . & . & . & $\mathrm{X}$ & . & $\mathrm{X}$ & $\mathrm{X}$ & $\mathrm{X}$ & $\mathrm{X}$ & $\mathrm{X}$ \\
\hline Pistillipollenites mcgregorii Range base is in NP9 & . & . & . &. &. & . & . &. & . & . & . & . & $\mathrm{X}$ & . & . & . & . \\
\hline Platycarya sp. A & . & . & . &. &. & . & . &. & . & . & . & . & . & $?$ & . & . & . \\
\hline Plicatopollis triorbicularis type & & . & . &. &. & . & . & . & & . & $\mathrm{X}$ & $\mathrm{X}$ & & . & . & . & . \\
\hline Pseudoplicapollis limitatus & & . & . &. &. & . & . & . & . & . & $\mathrm{X}$ & . & $\mathrm{X}$ & . & . & . & . \\
\hline Psilodiporites iszkaszentgyorgyi Range base is up. NP8 & . & . & . & . &. & . & . & . & . & . & . & . & . & prob. & . & . & \\
\hline Sparganiaceaepollenites sp. & . & . & . & . &. & . & . & . & . & . & . & . & . &. & . & . & $\mathrm{X}$ \\
\hline Thomsonipollis magnificus & . & . & . & . & . & . & . & . & . & . & $\mathrm{X}$ & . & . & . & . & . & $\mathrm{x}$ \\
\hline Tricolpites asper & . & . & . &. &. & . & . & . & . & . & . & . &. & . & . & $\mathrm{x}$ & . \\
\hline Ttricolpites crassus & & . & . &. &. & & . & & & . & & . & & . & . & $\mathrm{X}$ & . \\
\hline Triporopollenites microgranulatus & . & . & . & . & . & . & . & . & . & . & . & . & $\mathrm{X}$ & . & . & . & . \\
\hline Trudopollis spp., including T. plenus & & . & . & . & . & . & . & . & & . & $\mathrm{X}$ & . & . & . & . & . & . \\
\hline Ulmipollenites krempii & . & . & . &. &. & . & . & . & . & . & 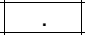 & . &. & $x$ & . & . & . \\
\hline Ulmipollenites tricostatus & & & . & . &. & & . & . & & . & $\mathrm{X}$ & . & . & . & . & . & . \\
\hline Ulmipollenites undulosus & & & & & & & & & & & & & . & . & & $\mathrm{X}$ & \\
\hline & & & & & & & & & & & & & & & & & \\
\hline prob. = probably belongs to this taxon. & & & & & & & & & & & & & & & & & \\
\hline & & & & & & & & & & & & & & & & & \\
\hline
\end{tabular}




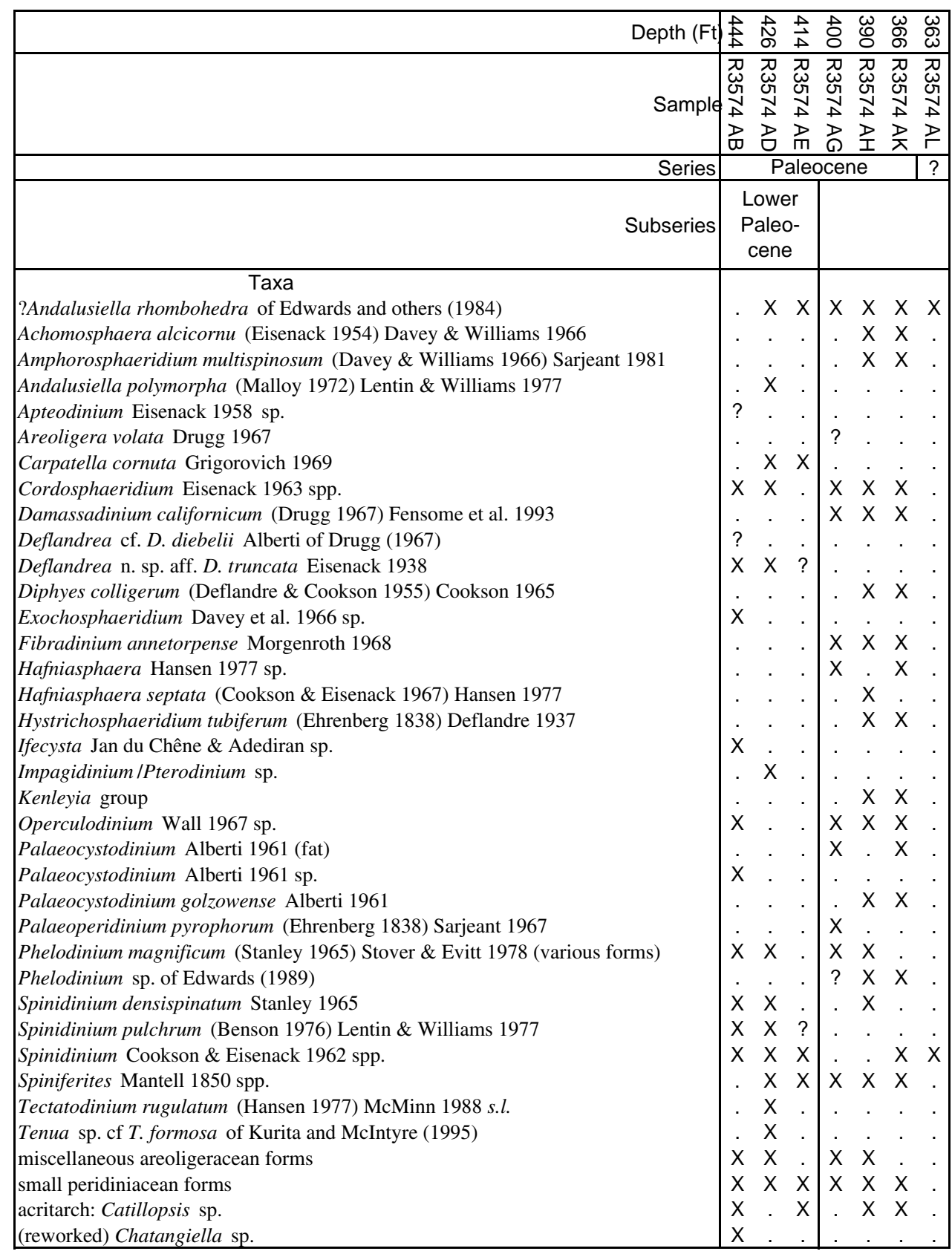

$\mathrm{X}=$ present, .=not present, ?=questionably present

Figure 8. Distribution of dinocyst taxa in Paleocene samples from the SRS P21TA corehole (BRN-335), Barnwell County, South Carolina. 


\begin{tabular}{|c|c|c|c|c|c|c|}
\hline Depth $(F t)$ & $\stackrel{\vec{P}}{\vec{P}}$ & $\stackrel{\vec{t}}{0}$ & $\begin{array}{l}\text { Nิ } \\
0 \\
0\end{array}$ & $\underset{⿱ ⺊ 尸}{\stackrel{P}{0}}$ & $\begin{array}{l}\omega \\
\infty \\
0\end{array}$ & $\underset{\sigma}{\sigma}$ \\
\hline Sample & 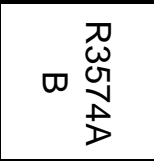 & 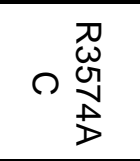 & $\nabla \underset{\mathbb{N}}{\stackrel{D}{N}}$ & 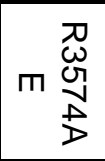 & 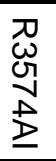 & 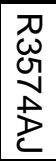 \\
\hline Age & 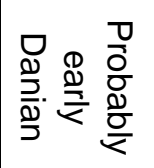 & 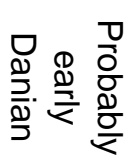 & 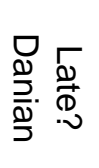 & 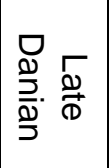 & 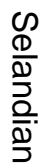 & $\begin{array}{l}\frac{\omega}{D} \\
\frac{D}{2} \\
\vdots \\
\frac{2}{2}\end{array}$ \\
\hline \multicolumn{7}{|l|}{ Pollen Taxa } \\
\hline Aesculiidites circumstriatus & . & . & . & . & $\mathrm{X}$ & $\mathrm{X}$ \\
\hline Alnus sp. & . & . & . & . & . & $\mathrm{x}$ \\
\hline Bombacacidites nanobrochatus & . & . & . & . & . & $x$ \\
\hline Bombacacidites paulus & . & $\mathrm{X}$ & . & . & . & . \\
\hline Bombacacides reticulatus & $\mathrm{X}$ & $\mathrm{X}$ & $\mathrm{X}$ & $\mathrm{X}$ & . & $\mathrm{x}$ \\
\hline Caryapollenites prodromus group & . & . & . & $\mathrm{x}$ & . & 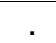 \\
\hline Choanopollenites conspicuus & . & . & . & . & $\mathrm{x}$ & $\mathrm{x}$ \\
\hline Favitricolporites baculoferus & . & $\mathrm{X}$ & $\mathrm{X}$ & $\mathrm{X}$ & . & \\
\hline Lanagiopollis cribellatus & . & . & . & . & . & $\mathrm{x}$ \\
\hline Milfordia minima & $\mathrm{X}$ & . & . & . & . & . \\
\hline Momipites coryloides/microfoveolatus & $\mathrm{X}$ & . & $\mathrm{X}$ & $\mathrm{X}$ & . & $\mathrm{X}$ \\
\hline Momipites dilatus & . & . & . & prob. & . & $x$ \\
\hline Momipites tenuipolus group & $\mathrm{x}$ & $\mathrm{X}$ & $\mathrm{X}$ & $\mathrm{X}$ & . & \\
\hline Nudopollis terminalis & $\mathrm{X}$ & $\mathrm{X}$ & $\mathrm{X}$ & . & . & $\mathrm{x}$ \\
\hline Nyssapollenites paleocenicus & $\mathrm{X}$ & $\mathrm{X}$ & & & $\mathrm{x}$ & $\mathrm{x}$ \\
\hline Osculapollis ? colporatus & $\mathrm{X}$ & $\mathrm{X}$ & $\mathrm{X}$ & $\mathrm{x}$ & . & $\mathrm{x}$ \\
\hline Plicatopollis triradiatus & . & . & $\mathrm{X}$ & . & . & \\
\hline Pseudoplicapollis limitatus & . & . & . & $\mathrm{X}$ & . & $\mathrm{x}$ \\
\hline Pseudoplicapollis serenus & $\mathrm{X}$ & . & $\mathrm{X}$ & $\mathrm{X}$ & . & . \\
\hline Tricolpites asper & 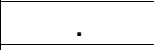 & $\mathrm{X}$ & . & . & . & . \\
\hline Tricolpites microreticulatus & $X ?$ & . & . & . & . & . \\
\hline Trudopollis spp., including T. plenus & & . & 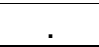 & . & . & $\mathrm{X}$ \\
\hline a & (6) & 11 & 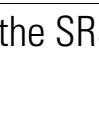 & $\Gamma \angle 1$ & & \\
\hline
\end{tabular}




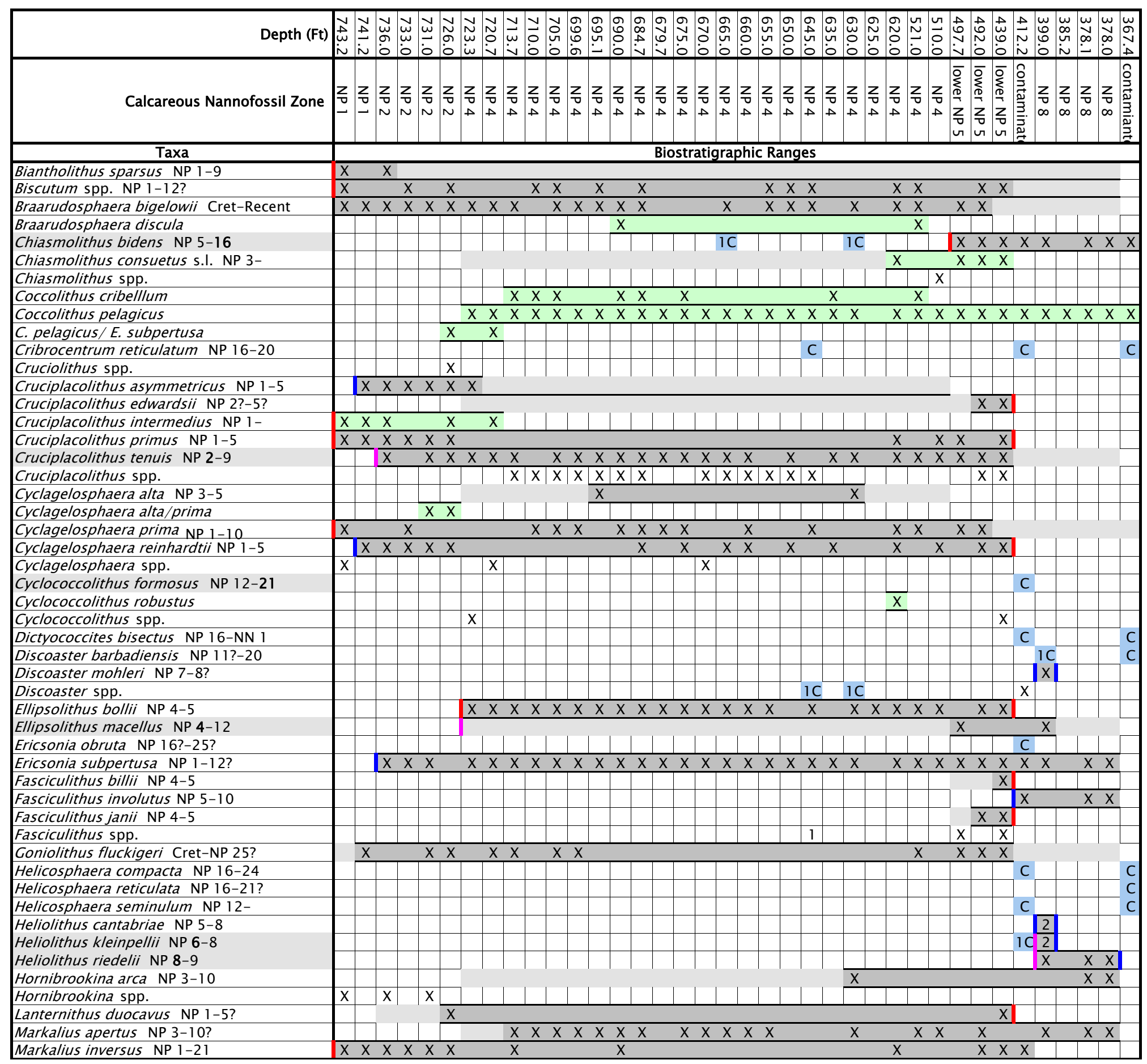




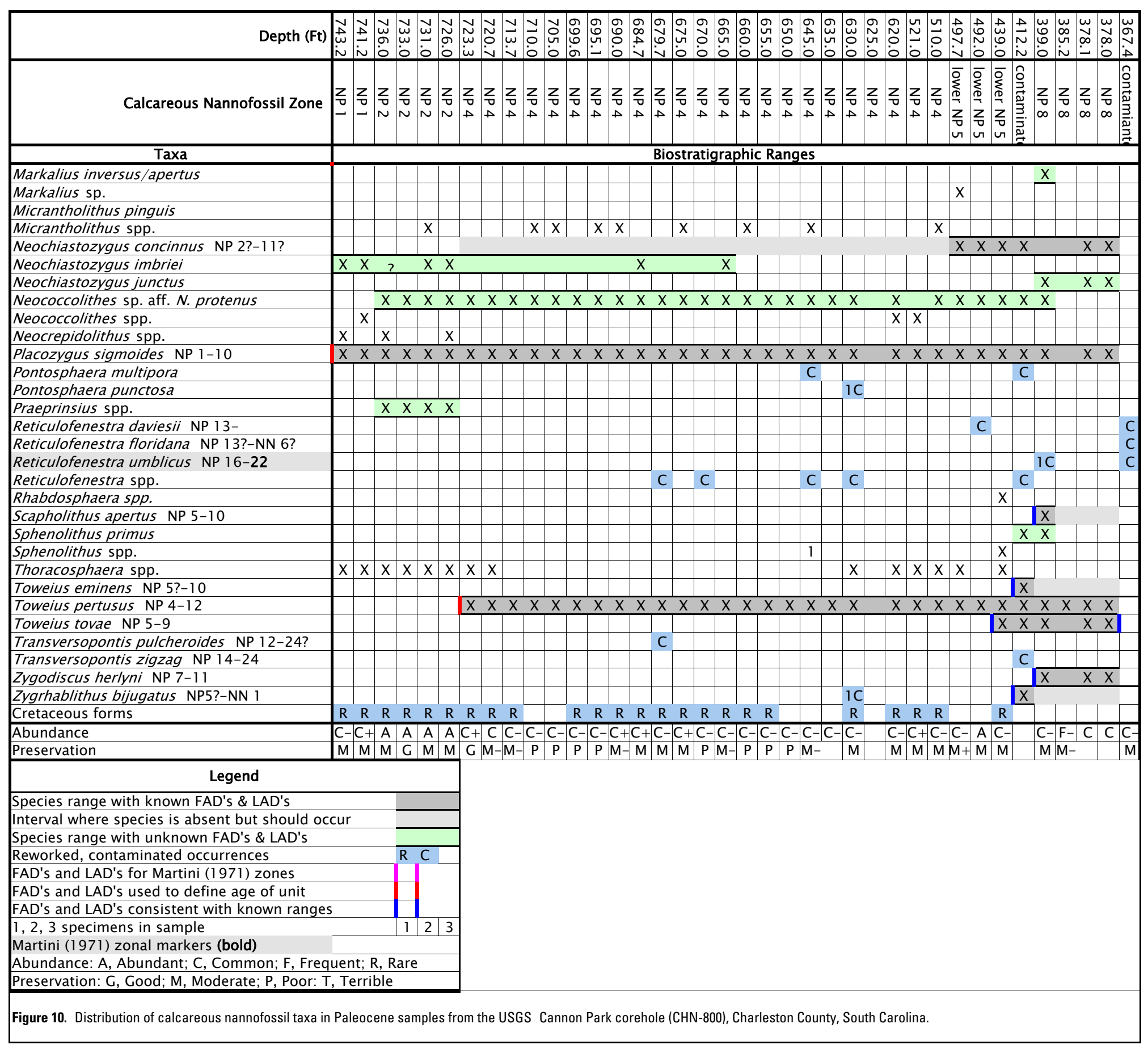




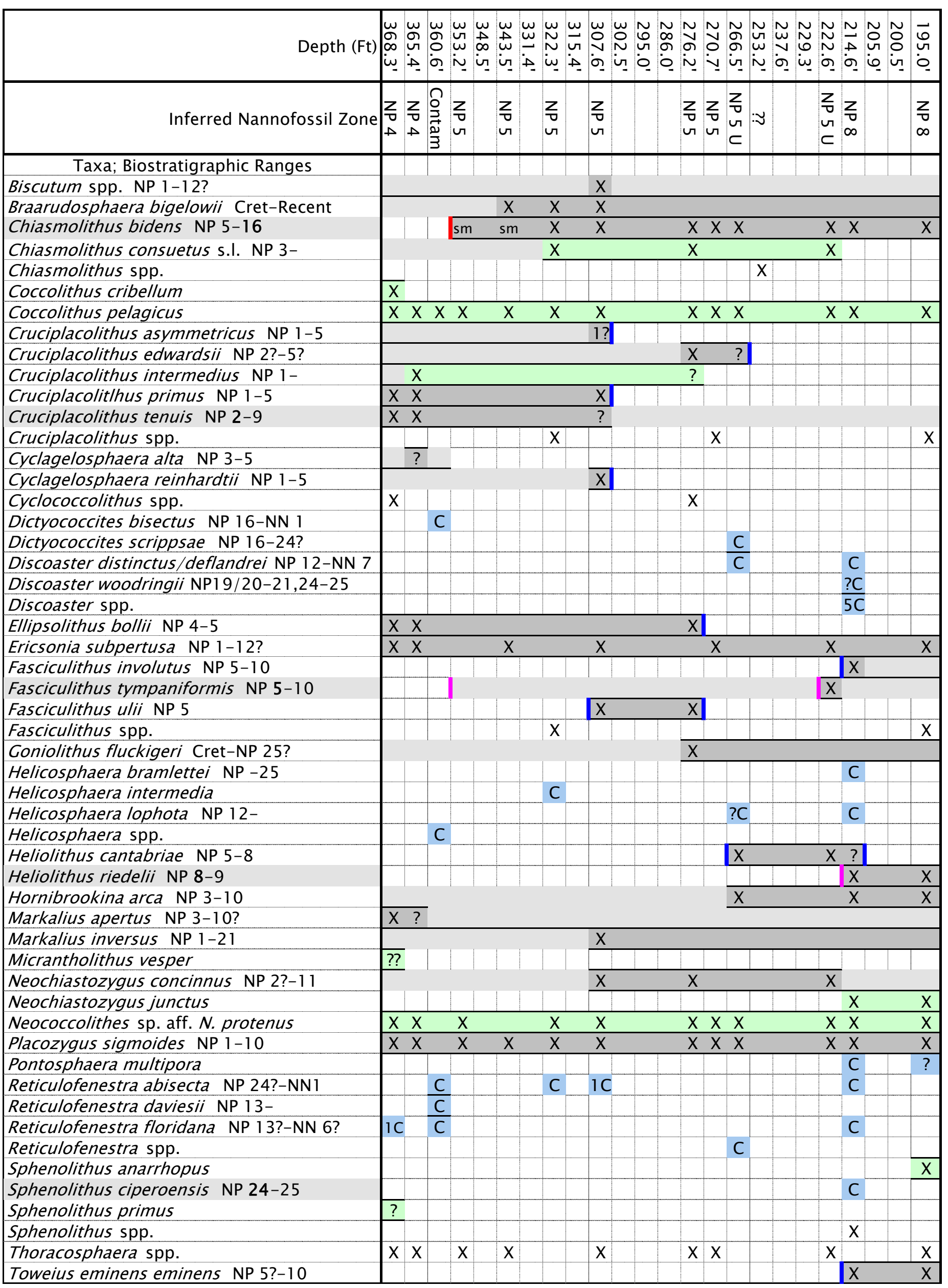




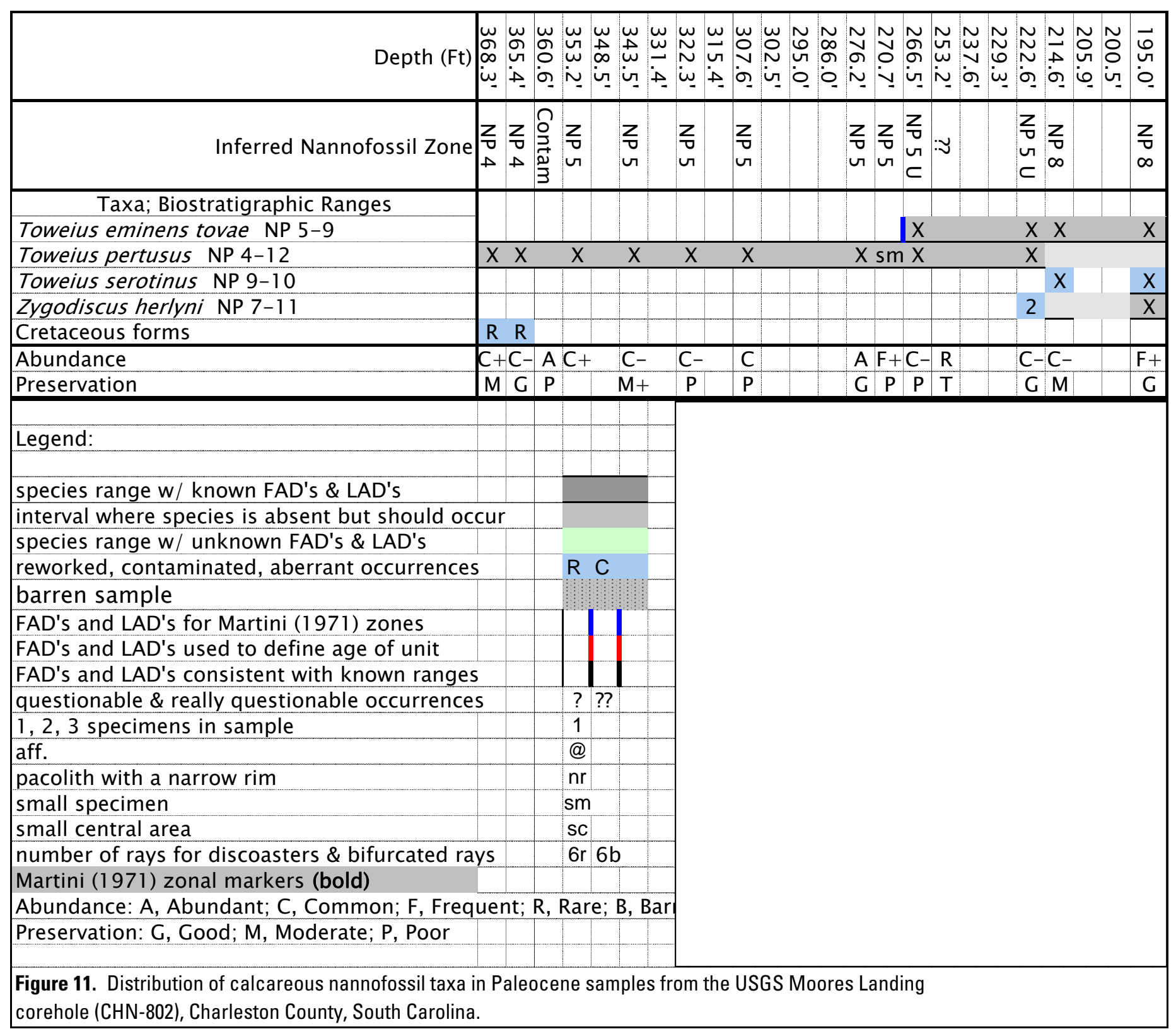




\begin{tabular}{|c|c|c|c|c|c|c|c|c|c|c|c|c|c|c|c|c|c|c|c|c|c|c|c|c|c|c|c|c|c|c|c|c|c|c|c|c|c|c|c|c|c|c|}
\hline Depth (Ft) & & & & & & & 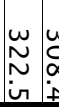 & & & & & & & 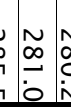 & & $\underset{\sim}{\tilde{N}} \underset{\sim}{\sim} \underset{\sim}{\sim}$ & & & & & & & \begin{tabular}{l|l}
$\sim$ & $\sim$ \\
$N$ & $w$ \\
& $\omega$ \\
0 & $\omega$ \\
\end{tabular} & & & & & & & & & & 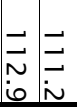 & $\begin{array}{l}\overrightarrow{8} \\
\\
\\
\end{array}$ & & & & & & & & \\
\hline Calcareous Nannofossil Zone & & $\begin{array}{l}z \\
z \\
n\end{array}$ & Z & $\begin{array}{l}Z \\
Z \\
\sim\end{array}$ & & $\begin{array}{ll}Z \\
\text { Z } \\
N\end{array}$ & $Z_{n}^{Z}$ & $\begin{array}{ll}Z_{0} & Z \\
\sim & \\
N\end{array}$ & $\begin{array}{ll}Z_{0} & Z \\
\sim & \end{array}$ & $\begin{array}{l}Z_{n} \\
\sim \\
N\end{array}$ & & & & 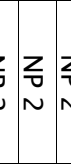 & & $\begin{array}{l}z \\
\mathbf{z} \\
\mathbf{z} \\
\mathbf{n}\end{array}$ & $\begin{array}{l}Z_{0} \\
Z_{0}\end{array}$ & & $\begin{array}{ll}z & Z \\
\sim & z \\
\sim & \end{array}$ & $\mid \begin{array}{l}z \\
z \\
A\end{array}$ & & & & 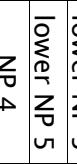 & 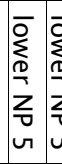 & & 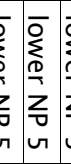 & 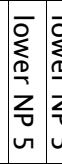 & & 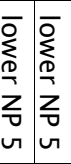 & 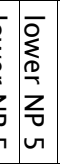 & 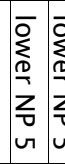 & 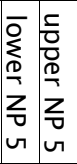 & $\begin{array}{l}z \\
u \\
u \\
0 \\
0 \\
\sigma \\
\sigma\end{array}$ & $\begin{array}{ll}z & \\
\bar{v} & z \\
v & z \\
o & \sigma \\
\vdots & \sigma\end{array}$ & & & & & $\approx$ & & u \\
\hline Taxa & & & & & & & & & & & & & & & & & & & & ostra & atig & grap & ohic R & Rang & ges & & & & & & & & & & & & & & & & & \\
\hline Biantholithus sparsus NP 1-9 & & & & & & & & & & & $\bar{X}$ & & & & & & & & & $\mathrm{X}$ & & & & & & & & & & & & & & & & & & & & & & \\
\hline Biscutum spp. NP 1-12? & & $\mathrm{X}$ & & & & & & & & & $\mathrm{X}$ & & & & & & & & & & & & & & & & & & & & & & & & & & & & & & & \\
\hline Braarudosphaera bigelowii Cret-Recent & $\mathrm{X}$ & $\mathrm{X}$ & $\mathrm{X}$ & $\mathrm{X}$ & $\mathrm{X}$ & $\mathrm{x}$ & $\mathrm{X}$ & & $x>$ & $\mathrm{x} x$ & $\mathrm{x} x$ & $\mathrm{xx}$ & $\mathrm{x} x$ & $\mathrm{x} x$ & & & & & $\mathrm{X}$ & $\mathrm{x}$ & & $\mathrm{X}$ & & & & $\mathrm{X}$ & $\mathrm{X}$ & 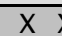 & $x \times$ & $\mathrm{XX}$ & $\bar{x}$ & & & & & & & & & & & \\
\hline Braarudosphaera discula & & & & & & & & & & & & & $\mathrm{X}$ & $\mathrm{X}$ & & & & & & 1 & & & & & & & & & & & & & & & & & & & & & & \\
\hline Braarudosphaera spp. & & & & & & & & & & & & & & $\mathrm{X}$ & $\mathrm{X}$ & & & & & & & & & & & & & & & & & & & & & & & & & & & \\
\hline Chiasmolithus bidens NP 5-16 & & & & & & & & & & & & & & 1 & & & & & & & & & & $\mathrm{X}$ & $\mathrm{X}$ & $\mathrm{XX}$ & $x$ & $\mathrm{X}$ & $\mathrm{xx}$ & $\mathrm{Xx}$ & $x \times$ & 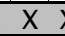 & $\mathrm{X}$ & & & $\bar{x}$ & & $\bar{x}$ & $\bar{x}$ & & & \\
\hline Chiasmolithus sp. aff C. bidens NP 4 & & & & & & & & & & & & & & & & & & & $\mathrm{X}$ & 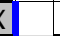 & & & & 1 & & & & & & & & & & & & & & & & & & \\
\hline Chiasmolithus consuetus s.I. NP 3- & & & & & & & & & & & 1 & & & 1 & & & & 1 & & & 1 & & & & $\mathrm{X}$ & & $\mathrm{X}$ & $\mathrm{X}$ & $\mathrm{xx}$ & $\mathrm{X}$ & & $\mathrm{X}$ & & & & & & & & & & \\
\hline Chiasmolithus spp. & & & & & & & & & & & & & & $\mathrm{x}$ & & & & & & & & & & & & & & & & & & & & $\mathrm{X}$ & & & & & & & & \\
\hline Coccolithus cribellum & & & & & & & & & & & & & & & & & & & ${ }^{*} x$ & & & & & $\mathrm{X}$ & & & & & & & & & & & & & & & & & & \\
\hline Coccolithus pelagicus & & & & 1 & & & $\mathrm{X}$ & & & & & & 1 & 2 & & & & & & $\mathrm{X}$ & $\mathrm{X}$ & $\mathrm{X}$ & $\mathrm{Xx}$ & $\mathrm{XX}$ & $\mathrm{X}$ & $\mathrm{XX}$ & $\mathrm{x} x$ & & $\mathrm{xx}$ & $\mathrm{XX}$ & $\mathrm{x} x$ & $x>$ & $\mathrm{XX}$ & $\bar{x}$ & $\mathrm{Xx}$ & $\bar{x}$ & & $\bar{x}$ & $\bar{x}$ & & & \\
\hline Cruciplacolithus asymmetricus NP 1-5 & & $\mathrm{X}$ & $\mathrm{X}$ & $\mathrm{X}$ & $\mathrm{X}$ & $\mathrm{X}$ & $\times>$ & $\overline{x>}$ & $\overline{x>}$ & $\overline{x x}$ & $\overline{x x}$ & $\mathrm{xx}$ & $\mathrm{Xx}$ & $x \times$ & $\mathrm{xx}$ & $\mathrm{X}$ & $\bar{x}$ & $\bar{x}$ & $\mathrm{X}$ & & & & $\mathrm{X}$ & & & & & $\mathrm{X}$ & & 1 & & & & & & & & & & & & \\
\hline Cruciplacolithus edwardsii NP 2?-5? & & & & & & & & & & & & & & 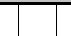 & & & & $? 1$ & & & & & & & & & & & & & & & & & & & & & & & & \\
\hline Cruciplacolithus intermedius NP 1- & $\mathrm{X}$ & $\mathrm{x}$ & & & $\mathrm{X}$ & $\mathrm{X}$ & & & & & & & $\bar{X}$ & & & & & & $\mathrm{X}$ & & & & $?$ & & & & & & & & & & & & & & & & & & & \\
\hline Cruciplacolit/hus primus NP 1-5 & $\mathrm{X}$ & $\mathrm{X}$ & $\mathrm{X}$ & $\mathrm{X}$ & $\mathrm{X}$ & $\mathrm{X}$ & $x>$ & $x>$ & $x>$ & $x>$ & $x>$ & $\mathrm{xx}$ & $\mathrm{Xx}$ & 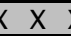 & $\mathrm{xx}$ & $\bar{x}$ & $x$ & $\mathrm{X}$ & $\bar{x}$ & 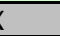 & & & & & & $\mathrm{X}$ & $\mathrm{X}$ & & & $\mathrm{X}$ & & & & & & & & & & & & \\
\hline Cruciplacolithus tenuis NP 2-9 & 1 & $\mathrm{X}$ & $\mathrm{X}$ & & & & & & & & & & & & & & & 1 & $\mathrm{X}$ & $\mathrm{x}$ & $\mathrm{X}$ & $\mathrm{X}$ & $\mathrm{Xx}$ & $\mathrm{Xx}$ & $\mathrm{X}$ & $\mathrm{X}$ & & & $\mathrm{X}$ & $\bar{x}$ & $\bar{x}$ & & & & & & & & & & & \\
\hline Cruciplacolithus spp. & & & & & & & & & & & & & & & & & & & & & & & & & & & & & & $\mathrm{X}$ & & $\mathrm{x} \mid \mathrm{r}$ & $\mathrm{X}$ & & & & & & & & & \\
\hline Cyclagelosphaer alta NP 3-5 & & & & & & & & & & & & & & & & & & & & & & & & & & & $?$ & & & & & & & & & & & & & & & \\
\hline Cyclagelosphaera prima NP 1-10 & $\mathrm{X}$ & $\mathrm{X}$ & & & & & $\mathrm{X}$ & & & & & & & & $\mathrm{X}$ & & & $\bar{X}$ & & & & & & & & & & & & & & & & & & & & & & & & \\
\hline Cyclagelosphaera reinhardtii NP 1-5 & & & & & & & $\mathrm{X}$ & & & & & & & & & & & & & & & & & & & & & & & & & & & & & & & & & & & \\
\hline Cyclagelosphaera spp. & & & & & & $\mathrm{x}$ & & & & $\mathrm{X}$ & & & $\mathrm{X}$ & $\mathrm{X}$ & & & & & & & & & & & & & & & & & $\mathrm{X}$ & & & & & & & & & & & \\
\hline Cyclococcolithus robustus & & & & & & & & & & & & & & & & & & & & & & & & & & & & & & & & & $?$ & & & & & & & & & \\
\hline Cyclococcolithus spp. & & & & & & & & & & & & & & & & & & & & & $\mathrm{X}$ & & & & $\mathrm{X}[\mathrm{\gamma}$ & $\mathrm{X}$ & & & & & & & & & & & & & & & & \\
\hline Ellipsolithus bollii NP 4-5 & & & & & & & & & & & & & & & & & & & & & & & & & $\mathrm{X}$ & 1 & & $\mathrm{X}$ & & $\mathrm{X}$ & & & & & & & & & & & & \\
\hline Ellipsolithus distichus NP 4-12 & & & & & & & & & & & & & & & & & & & & 1 & & & & & & & & & & & & & & & & & & & & & & \\
\hline Ellipsolithus macellus NP 4-12 & & & & & & & & & & & & & & 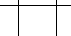 & & & & & & & $\mathrm{X}$ & $\mathrm{x}$ & $\mathrm{x}$ & & & & & & & & & & & & & & & & & & & \\
\hline Ericsonia subpertusa NP 1-12? & & $\mathrm{x}$ & $\mathrm{X}$ & $\mathrm{x}$ & $\mathrm{X}$ & $\mathrm{x}$ & $x>$ & $x>$ & $\mathrm{X}$ & $x>$ & $\overline{x>}$ & $\mathrm{xx}$ & $x \times$ & $x \times$ & $x \times$ & $\bar{x}$ & $\bar{x})$ & $\bar{x})$ & $\mathrm{x} x$ & $x$ & $\mathrm{X}$ & $\mathrm{X}$ & $\mathrm{Xx}$ & $\mathrm{XX}$ & $x>$ & $\mathrm{X} ?$ & $? \mathrm{X}$ & & & $\mathrm{XX}$ & $\mathrm{x} x$ & $x$ & $\mathrm{X}$ & & & $\bar{X}$ & & $\bar{x}$ & & & & \\
\hline Fasciculithus involutus NP 5-10 & & & & & & & & & & & & & & & & & & & & & & & & & & & & & & & & & & & & & & $\underline{X}$ & & & & \\
\hline Fasciculithus spp. & & & & & & & & & & & & & & & & & & & & & & & & & & & $\mathrm{X}$ & $\mathrm{X}$ & $\mathrm{X}$ & & & & & & & & & & & & & \\
\hline Goniolithus fluckigeri Cret-NP 25? & & & $\mathrm{X}$ & & & & $\mathrm{X}$ & & & & & & & $\mathrm{X}$ & & & & & $\mathrm{X}$ & & & & & & $\mathrm{X}$ & & & $\mathrm{x}$ & & $\mathrm{x} x$ & $\bar{x}$ & & & & & & & & & & & \\
\hline Heliolithus cantabriae NP 5-8 & & & & & & & & & & & & & & & & & & & & & & & & & & & & & & & & & 1 & & & & & $\mathrm{x}$ & & & & \\
\hline Heliolithus kleinpellii NP 6-8 & & & & & & & & & & & & & & & & & & & & & & & & & & & & & & & & & & & & $\bar{X}$ & & $\bar{x}$ & & & & \\
\hline Heliolithus spp. & & & & & & & & & & & & & & & & & & & & & & & & & & ?? & & & & & & & & & & & & & & & & \\
\hline Hornibrookina spp. & & & & $\mathrm{X}$ & $\mathrm{X}$ & & & & & 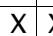 & $\bar{x} \mid \lambda$ & \begin{tabular}{l|l}
$X$ & $X$ \\
\end{tabular} & \begin{tabular}{l|l}
$\mathrm{X}$ & $\mathrm{X}$ \\
$\mathrm{n}$
\end{tabular} & \begin{tabular}{l|l|}
$X$ & $X$ \\
\end{tabular} & & $\mathrm{X}$ & & $\mathrm{X}$ & & & & & & & & & & & & & & & & & & & & & & & & \\
\hline Lanternithus duocavus NP 1-5? & & & & & & & & & & & & & & $x$ & & & & & & & & & & & & & & & & & & & & & & & & & & & & \\
\hline Lanternithus sp. & & & & & & & $?$ & & & & & & & & & & & & & & & & & & & & & & & & & & & & & & & & & & & \\
\hline Markalius apertus NP 3-10? & & & & & & & & & & & & & & & & & & & & & & & & & & & & & & & $?$ & & & & & & & & & & & \\
\hline Markalius inversus NP $1-21$ & $\mathrm{X}$ & $\mathrm{x}$ & $\mathrm{X}$ & $\mathrm{X}$ & & $\mathrm{x}$ & $\mathrm{x}$ & & & $\mathrm{X}$ & & $\mathrm{xx}$ & $\bar{X}$ & & & & $\bar{X}$ & & & & & $\mathrm{X}$ & $\mathrm{Xx}$ & & & & & & & & & & & & & & & & & & & \\
\hline Micrantholithus aequalis & & & & & & & & & 1 & & & & & & & & & & & & & & & & & & & & & & & & & & & & & & & & & \\
\hline Micrantholithus fornicatus & & & & & & & & & & & & & $\mathrm{X}$ & X & & & & & & & & & & & & & & & & & & & & & & & & & & & & \\
\hline Micrantholithus pinguis & & & & & & & & & & & $\mathrm{X}$ & & $\mathrm{X}$ & & & & & & & & & & & & & & & & & & & & & & & & & & & & & \\
\hline Micrantholithus vesper & & & & & & & & & & & & & & & & & & & & & & & & & $?$ & & & & & & & & & & & & & & & & & \\
\hline Micrantholithus spp. & & & & & & $\mathrm{X}$ & & & & $\mathrm{X}$ & & & & & & & & & $\mathrm{X}$ & & & & & & & $\mathrm{X}$ & & & & & & & & & & & & & & & & \\
\hline Neochiastozygus concinnus NP 2?-11? & & & & & & & & & & & & & & & & & & & & & & & & & & & & $\bar{x}$ & & $\mathrm{Xx}$ & & $\bar{x}$ & & & & $\bar{X}$ & $?$ & $\bar{X}$ & & & & \\
\hline Neococcolithes sp. aff. N. protenus & & & $\mathrm{X}$ & & $\mathrm{X}$ & $x$ & & & & & & & & & & & & & $\mathrm{X}$ & $x$ & $\mathrm{X}$ & $\mathrm{X}$ & $\mathrm{X}$ & $\mathrm{X}$ & 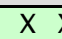 & $\mathrm{X}$ & $\mathrm{X}$ & $\mathrm{x}$ & $\mathrm{X}$ & & $\mathrm{X}$ & $\mathrm{x}$ & $\mathrm{X}$ & & & $\mathrm{X}$ & & $\mathrm{X}$ & $\bar{x}$ & & & \\
\hline Neococcolithes spp. & $\mathrm{X}$ & $\mathrm{X}$ & & $\mathrm{X}$ & & & & & $\mathrm{X}$ & & \begin{tabular}{l|l}
$X$ & $\gamma$
\end{tabular} & \begin{tabular}{l|l}
$X$ \\
$X$
\end{tabular} & $\mathrm{X}$ & $\mathrm{X} \mid \mathrm{r}$ ( & $\mathrm{X} \mid \mathrm{X}$ & $\mathrm{X}$ & $\mathrm{X}[\mathrm{r}$ & $\mathrm{X} \mid \mathrm{x}$ & \begin{tabular}{l|l}
$X$ & $X$ \\
\end{tabular} & & & & & & & & & & & & & & & & & & & & & & & \\
\hline
\end{tabular}




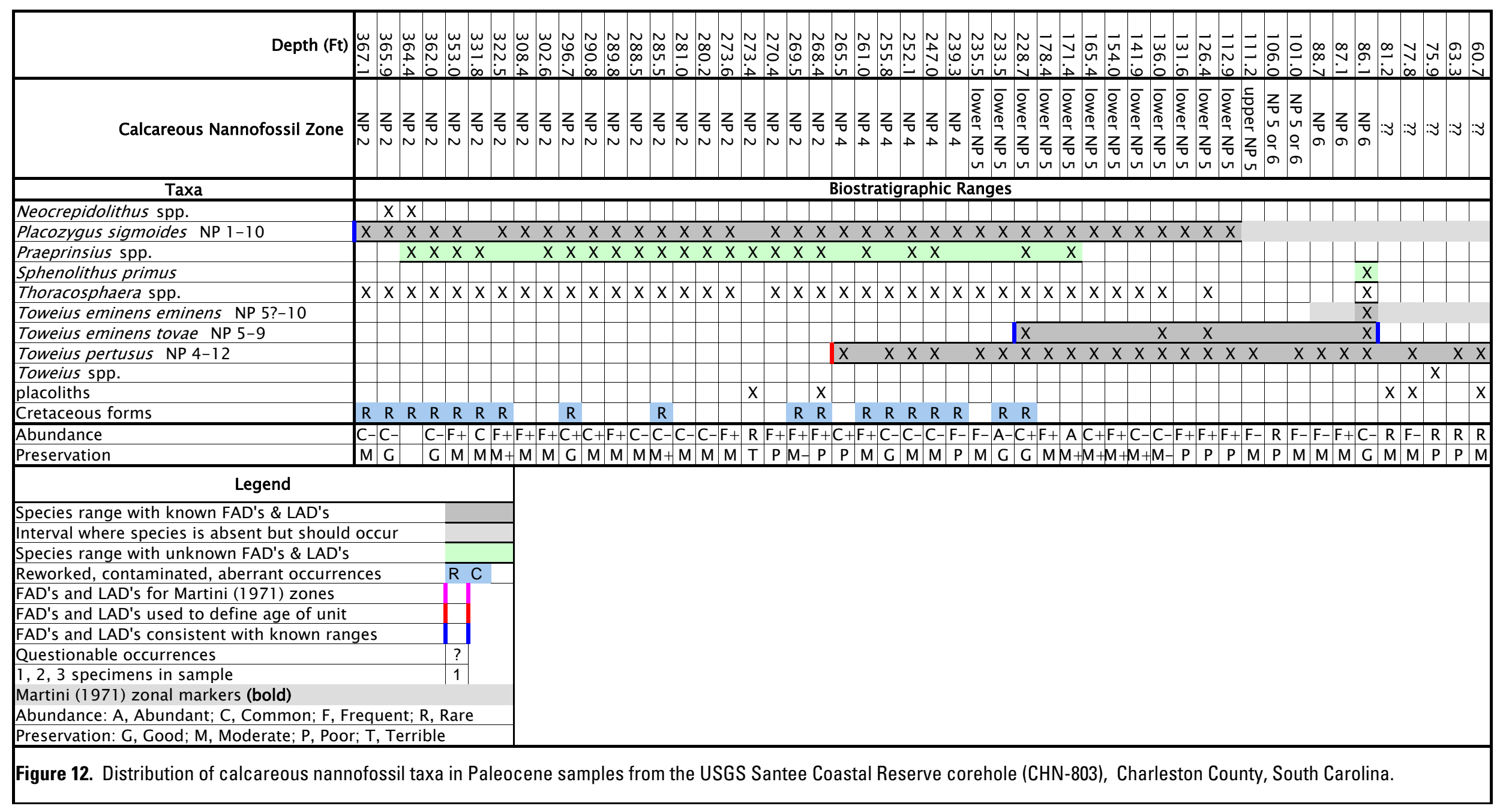




\begin{tabular}{|c|c|c|c|c|c|c|}
\hline Depth $(\mathrm{Ft})$ & $\begin{array}{l}0 \\
N \\
N \\
\dot{1} \\
\dot{0} \\
N \\
N \\
\dot{D} \\
\end{array}$ & $\begin{array}{l}0 \\
0 \\
+ \\
0 \\
0 \\
0 \\
! \\
\omega \\
\omega\end{array}$ & $\begin{array}{l}0 \\
\tilde{N} \\
\omega \\
0 \\
0 \\
0 \\
\tilde{\omega} \\
\omega \\
\omega \\
\end{array}$ & $\begin{array}{l}1 \\
N \\
0 \\
0 \\
0 \\
N \\
0 \\
0 \\
D\end{array}$ & 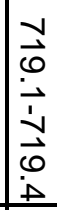 & \\
\hline Sample & $\begin{array}{l}D \\
O \\
\stackrel{1}{N} \\
N \\
D\end{array}$ & 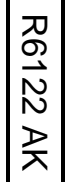 & 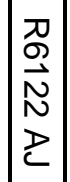 & $\begin{array}{l}D \\
D \\
\stackrel{D}{N} \\
N \\
\geq \\
\geq\end{array}$ & 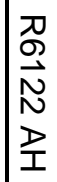 & \\
\hline Series & \multicolumn{6}{|c|}{ Paleocene } \\
\hline Subseries & \multicolumn{5}{|c|}{ upper } & \\
\hline \multicolumn{7}{|l|}{ Taxa } \\
\hline ?Andalusiella rhombohedra of Edwards and others (1984) & $\mathrm{X}$ & $\mathrm{X}$ & . & . & . & \\
\hline Cordosphaeridium fibrospinosum Davey \& Williams 1966 & $\mathrm{X}$ & & 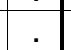 & . & $\mathrm{X}$ & \\
\hline Damassadinium californicum (Drugg 1967) Fensome et al. 1993 & $?$ & $\mathrm{X}$ & $\mathrm{X}$ & $\mathrm{X}$ & $\mathrm{X}$ & \\
\hline Deflandrea delineata Cookson \& Eisenack 1965 & $?$ & $\mathrm{X}$ & $\mathrm{X}$ & $\mathrm{X}$ & . & \\
\hline Exochosphaeridium Davey et al. 1966 sp. & $\mathrm{X}$ & . & . & . & . & \\
\hline Fibradinium annetorpense Morgenroth 1968 & $\mathrm{X}$ & . & . & . & . & \\
\hline Fibrocysta Stover \& Evitt 1978 sp. & $\mathrm{X}$ & . & . & . & . & \\
\hline Fromea fragilis (Cookson \& Eisenack 1962) Stover \& Evitt 1978 & 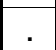 & . & . & . & $\mathrm{X}$ & \\
\hline Hystrichokolpoma unispinum Williams \& Downie 1966 & 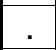 & . & . & . & $\mathrm{X}$ & \\
\hline Impagidinium Stover \& Evitt 1978 spp. & $\mathrm{X}$ & . & . & . & . & \\
\hline Kallosphaeridium brevibarbatum de Coninck 1969 & & . & . &. & $\mathrm{x}$ & \\
\hline Kallosphaeridium De Coninck 1969 spp. & $\mathrm{X}$ & & . &. & . & \\
\hline Nematosphaeropsis Deflandre \& Cookson 1955 sp. & . & $?$ & . & . & $\mathrm{X}$ & \\
\hline Operculodinium Wall 1967 spp. & $\mathrm{X}$ & & . & . & $\mathrm{X}$ & \\
\hline Palaeocystodinium golzowense Alberti 1961 & $\mathrm{X}$ & $\mathrm{X}$ & . & $\mathrm{X}$ & . & \\
\hline Phelodinium Stover \& Evitt 1978 sp. & $\mathrm{X}$ & & . & . & . & \\
\hline Phelodinium sp. of Edwards (1989) & $\mathrm{X}$ & $\mathrm{X}$ & . & $\mathrm{X}$ & . & \\
\hline Spinidinium Cookson \& Eisenack 1962 spp. & $\mathrm{X}$ & . & . & . & . & \\
\hline Spiniferites mirabilis (Rossignol 1964) Sarjeant 1970 & & . & . & . & . & \\
\hline Spiniferites pseudofurcatus (Klumpp 1953) Sarjeant 1970 & $\mathrm{X}$ & . & . &. & . & \\
\hline Spiniferites Mantell 1850 spp. & $\mathrm{X}$ & $\mathrm{X}$ & . & $\mathrm{X}$ & $\mathrm{X}$ & \\
\hline Turbiosphaera filosa (Wilson 1967) Archangelsky 1969 & & & . & $\mathrm{X}$ & . & \\
\hline miscellaneous areoligeracean forms & $X$ & $\mathrm{X}$ & . & . & $\mathrm{X}$ & \\
\hline miscellaneous cladopyxidiacean forms & & . & . & $\mathrm{X}$ & . & \\
\hline small peridiniacean forms & $\mathrm{X}$ & & . & $\mathrm{X}$ & $\mathrm{X}$ & \\
\hline \multicolumn{7}{|l|}{$\mathrm{X}=$ present, .=not present, ?=questionably present } \\
\hline $\begin{array}{l}\text { Figure 13. Distribution of dinocyst taxa in Paleocene samples from the } \\
\text { USGS/DNR Edisto Island corehole (COL-364), Colleton County, South Carolina. }\end{array}$ & & & & & & \\
\hline
\end{tabular}




\begin{tabular}{|c|c|c|c|c|c|c|c|c|c|c|c|c|c|c|c|c|c|c|c|c|c|}
\hline Depth $(\mathrm{Ft})$ & & & & & & & & & & & $\mid \begin{array}{l}\infty \\
w \\
\infty \\
\sim \\
\sim\end{array}$ & & & & & & & & \begin{tabular}{l|l}
$N$ & $N$ \\
$\tilde{N}$ & $\tilde{w}$ \\
0 & $\infty$
\end{tabular} & & 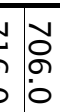 \\
\hline Calcareous Nannofossil Zone & 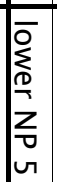 & & & & & & & 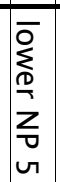 & & 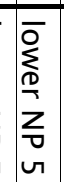 & $\mid \begin{array}{l}\bar{D} \\
\sum_{0} \\
\underline{D} \\
z \\
z \\
v \\
v\end{array}$ & 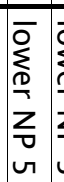 & & & & 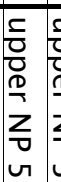 & $\begin{array}{l}\frac{5}{0} \\
\bar{D} \\
\mathbb{D} \\
Z \\
z \\
v \\
v\end{array}$ & & & & \begin{tabular}{l|l}
$z$ \\
$\vdots$ \\
0 \\
0
\end{tabular} \\
\hline Taxa & & & & & & & & & $\overline{\text { ostra }}$ & ratig & igrap & $\overline{\text { phic }}$ & $\mathrm{c} \mathrm{Ra}$ & $\overrightarrow{a n g}$ & & & & & & & \\
\hline Biscutum spp. NP 1-12? & & $\mathrm{X}$ & & & & & & & & & & & & & & & & & & & \\
\hline Blackites spp. & & & & & & & & & & & & & & & & & & & & & C \\
\hline Braarudosphaera bigelowii Cret-Recent & $\mathrm{X}$ & & & $\mathrm{X}$ & $\mathrm{X}$ & $\mathrm{x}$ & $\mathrm{x}$ & $\mathrm{x}$ & $\mathrm{X}$ & $\mathrm{x}$ & $\mathrm{x}$ & $\mathrm{x}$ & $\mathrm{x}$ & & & & & & $\mathrm{X}$ & & $\mathrm{x}$ \\
\hline Campylosphaera dela NP 9-17 & & & & & & & & & & & & & & & & & & & & & \\
\hline Cepekiella lumina NP 11-24? & & & & & & & & & & & & & & & & & & & & & 10 \\
\hline Chiasmolithus bidens NP 5-16 & $\bar{x}$ & $\mathrm{X}$ & $\mathrm{X}$ & $\mathrm{X}$ & $\mathrm{X}$ & $\mathrm{X}$ & $\mathrm{X}$ & $\mathrm{X}$ & $\mathrm{X}$ & $\mathrm{X}$ & $\mathrm{X}$ & $\mathrm{x}$ & $\mathrm{X}$ & $\mathrm{X}$ & & & & & $\mathrm{X}$ & & $x \times$ \\
\hline Chiasmolithus consuetus s.I. NP 3- & & & & $\mathrm{X}$ & $\mathrm{X}$ & & & $\mathrm{X}$ & & & & $\mathrm{X}$ & & & & & & & $\bar{X}$ & & $\bar{x}$ \\
\hline Coccolithus pelagicus & & $\bar{x}$ & $\mathrm{X}$ & $\mathrm{X}$ & $\mathrm{X}$ & $\mathrm{X}$ & $\mathrm{X}$ & $\mathrm{X}$ & $\mathrm{X}$ & $\mathrm{X}$ & $\mathrm{X}$ & $\mathrm{X}$ & $\mathrm{X}$ & & & $\mathrm{X}$ & $\mathrm{X}$ & $\mathrm{X}$ & $\mathrm{X}$ & & $\mathrm{xx}$ \\
\hline Cruciplacolithus asymmetricus NP 1-5 & $\mathrm{X}$ & $\mathrm{X}$ & & & $\mathrm{X}$ & $\mathrm{x}$ & & & & & & & & & & & & & & & \\
\hline Cruciplacolithus primus NP 1-5 & & & $\mathrm{X}$ & $\mathrm{X}$ & & & & & & & $\mathrm{X}$ & & & & & & & & & & \\
\hline Cruciplacolithus tenuis NP 2-9 & $\mathrm{X}$ & & & $\mathrm{X}$ & $\bar{X}$ & & & & $\bar{X}$ & & $\bar{x}$ & & & & & & & & & & \\
\hline Cruciplacolithus spp. & & & $\mathrm{X}$ & & $\mathrm{X}$ & $\mathrm{X}$ & & $\mathrm{X}$ & & $\mathrm{X}$ & & & & & & & & $\mathrm{X}$ & & & $x \mid x$ \\
\hline Cyclagelosphaera spp. & $x$ & & $\mathrm{X}$ & & & & & & & & & $\mathrm{X}$ & $\mathrm{X}$ & & & & & & & & \\
\hline Cyclococcolithus spp. & $\mathrm{X}$ & & $\mathrm{X}$ & $\mathrm{X}$ & $\mathrm{X}$ & $\mathrm{X}$ & $\mathrm{X}$ & & & $\mathrm{X}$ & $\mathrm{X}$ & & $\mathrm{X}$ & & & & & & & & \\
\hline Discoaster mohleri NP 7-8? & & & & & & & & & & & & & & & & & & & $\mathrm{X}$ & & \\
\hline Discoaster spp. & & & & 1C & & & & & & & & & & & & & & & & & \\
\hline Ellipsolithus bollii NP 4-5 & & & $\bar{X}$ & $\mathrm{x}$ & $\mathrm{X}$ & & $\mathrm{X}$ & & $\mathrm{X}$ & $\mathrm{x}$ & $\mathrm{x}$ & $\mathrm{x}$ & $\mathrm{X}$ & & & & & & & & \\
\hline Ellipsolithus distichus NP 4-12 & & & & & & $\mathrm{X}$ & $\mathrm{X}$ & & & & & & & & & & & & $\bar{x}$ & & $\bar{x}$ \\
\hline Ellipsolithus macellus NP 4-12 & & & & & & $\mathrm{X}$ & & $\mathrm{X}$ & & $\mathrm{X}$ & & $\mathrm{X}$ & $\bar{X}$ & & & & & & & & \\
\hline Ericsonia subpertusa NP 1-12? & $\mathrm{X}$ & $\bar{x}$ & $\bar{X}$ & $\mathrm{X}$ & $\mathrm{X}$ & $\mathrm{x}$ & $\mathrm{X}$ & $\mathrm{X}$ & $\mathrm{X}$ & $\mathrm{X}$ & $\mathrm{X}$ & $\mathrm{X}$ & $\bar{X}$ & & & $\bar{X}$ & & & $\bar{X}$ & & $\mathrm{xx}$ \\
\hline Fasciculithus involutus NP 5-10 & & & & & & & & & & $\mathrm{X}$ & & $\mathrm{X}$ & & & & & & & $\mathrm{X}$ & & \\
\hline Fasciculithus ulii NP 5 & & & $\mathrm{X}$ & $\mathrm{X}$ & $\mathrm{X}$ & $\mathrm{X}$ & & $\mathrm{X}$ & & & & $\mathrm{X}$ & $\mathrm{X}$ & & & & & & & & \\
\hline Fasciculithus spp. & & & & & & & & & & & $\bar{X}$ & & & & & & & $\mathrm{X}$ & & & \\
\hline Goniolithus fluckigeri Cret-NP 25? & $\mathrm{X}$ & $x$ & & & & & & & & & & & & & & & & & & & \\
\hline Heliolithus cantabriae NP 5-8 & & & & & & & & & & & & & & & & $\mathrm{X}$ & & & $\mathrm{X}$ & & \\
\hline Heliolithus riedelii NP 8-9 & & & & & & & & & & & & & & & & & & $\bar{X}$ & $\bar{X}$ & & \\
\hline Hornibrookina arca NP 3-10 & & & & & & & & & & & & & & & & & & $\mathrm{X}$ & $\mathrm{X}$ & & \\
\hline Isthmolithus recurvus NP 19/20-21 & & & & & & & & & & & & & & & & & & & & & 10 \\
\hline Lophodolithus spp. & & & & & & & & & & & & & & & & & & & & & ? \\
\hline Markalius apertus NP 3-10? & $\mathrm{X}$ & & & & $\mathrm{X}$ & & & & & $\mathrm{X}$ & & & $\mathrm{X}$ & & & & & & $\mathrm{X}$ & & $\mathrm{x}$ \\
\hline Markalius inversus NP 1-21 & & & & & $\mathrm{X}$ & $\mathrm{X}$ & & $\bar{X}$ & $\mathrm{X}$ & & & & & & & & & & & & \\
\hline Micrantholithus inaequalis & & & & & & & & & & & & & & & & & & & $?$ & & \\
\hline Micrantholithus pinguis & & & & & & & & & & & & & & & & & & $?$ & & & \\
\hline Micrantholithus spp. & $\mathrm{X}$ & & & $X$ & & & $X$ & & & & & & & & & & & & & & $\mathrm{x}$ \\
\hline Neochiastozygus concinnus NP 2?-11 & & $\bar{x}$ & $\bar{X}$ & & & $\bar{x}$ & & $\mathrm{X}$ & $\mathrm{X}$ & $\mathrm{X}$ & $\mathrm{x}$ & $\mathrm{X}$ & $\mathrm{X}$ & & & & $\bar{x}$ & $\bar{x}$ & & & $\bar{x}$ \\
\hline Neochiastozygus junctus & & & & & & & & & & & & & & & & & & & $\mathrm{X}$ & & \\
\hline Neococcolithes sp. aff. N. protenus & $\mathrm{X}$ & $\mathrm{X}$ & $\mathrm{X}$ & $\mathrm{X}$ & $\mathrm{X}$ & $x$ & & $\mathrm{X}$ & $\mathrm{X}$ & $\mathrm{X}$ & $\mathrm{X}$ & $\mathrm{X}$ & $\bar{X}$ & & & $\mathrm{X}$ & & $\bar{X}$ & $\bar{X}$ & & $\mathrm{xx}$ \\
\hline Neocrepidolithus spp. & & & & & $\mathrm{X}$ & & & $\mathrm{X}$ & & & & & & & & & & & & & \\
\hline Pemma spp. & & & 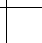 & & & & & & & & & 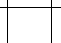 & . & & & & & . & 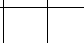 & & $?$ \\
\hline Placozygus sigmoides NP 1-10 & $\mathrm{X}$ & $\mathrm{X}$ & $\mathrm{X}$ & $\mathrm{X}$ & $\mathrm{X}$ & $\mathrm{X}$ & $\mathrm{X}$ & $\mathrm{X}$ & $\mathrm{X}$ & $\mathrm{X}$ & $\mathrm{X}$ & $\mathrm{X}$ & $\bar{X}$ & & & & & $\bar{X}$ & $\mathrm{X}$ & & \\
\hline Pontosphaera spp. & & & & & $\mathrm{X}$ & & & & & & & & & & & & & & & & \\
\hline Praeprinsius spp. & & & $\mathrm{X}$ & & & & & & & & & & & & & & & & & & \\
\hline Reticulofenestra umblicus NP 16-22 & & & & & & & & & & & & & & & & & & & & & 10 \\
\hline Reticulofenestra spp. & & & & & & & & & & & & C & & & & & & & & & \\
\hline Scapholithus apertus NP 5-10 & & & & & & & & & & & & & & & & & & & $\mathrm{X}$ & & $\bar{x}$ \\
\hline Sphenolithus anarrhopus & & & & & & & & & & & & & & & & & & & $\bar{X}$ & & \\
\hline Sphenolithus primus/moriformis & & & & & $\bar{X}$ & & & & & $\bar{x}$ & & $\bar{x}$ & & & & & & & $\bar{X}$ & & $\bar{x}$ \\
\hline Sphenolithus spp. & & & & & & & $\mathrm{X}$ & & & & & & $?$ & & & & & & & & \\
\hline Thoracosphaera spp. & $\mathrm{X}$ & $\mathrm{X}$ & $\mathrm{X}$ & $\mathrm{X}$ & $\mathrm{X}$ & $\mathrm{X}$ & $\mathrm{X}$ & $\mathrm{X}$ & $\mathrm{X}$ & $\mathrm{X}$ & $\mathrm{X}$ & $\mathrm{X}$ & & & & & & & $\mathrm{X}$ & & \\
\hline Toweius eminens eminens NP 5?-10 & & & & & & & & & & & & & & & & & & & $x$ & & \\
\hline Toweius eminens tovae NP 5-9 & & & & & & & & & $\mathrm{X}$ & $\mathrm{X}$ & & & & & & & & $\mathrm{X}$ & $\mathrm{X}$ & & $\mathrm{x} x$ \\
\hline Toweius pertusus NP 4-12 & $\mathrm{X}$ & & $\mathrm{X}$ & $\mathrm{X}$ & $\mathrm{X}$ & $\mathrm{x}$ & $\mathrm{X}$ & $\mathrm{x}$ & $\mathrm{X}$ & $\mathrm{X}$ & $\mathrm{X}$ & $\mathrm{X}$ & $\bar{X}$ & & $\bar{X}$ & $\mathrm{X}$ & $\mathrm{X}$ & $\bar{x}$ & $\mathrm{XX}$ & $\mathrm{X}$ & $\mathrm{xx}$ \\
\hline Toweius serotinus NP 9-10 & & & & 10 & & & & & & & & & & & & & & & $?$ & & \\
\hline Transversopontis pulcher NP 9-17? & & & & & & & & & & & & $\mathrm{C}$ & & & & & & & & & 2( \\
\hline Zygodiscus herlyni NP 7-11 & & & & & & & & & & & & & & & & & & $\mathrm{X}$ & $\mathrm{X}$ & & $x x$ \\
\hline placoliths & & & & & & & & & & & & & & $\mathrm{X}$ & & & & & & & \\
\hline
\end{tabular}




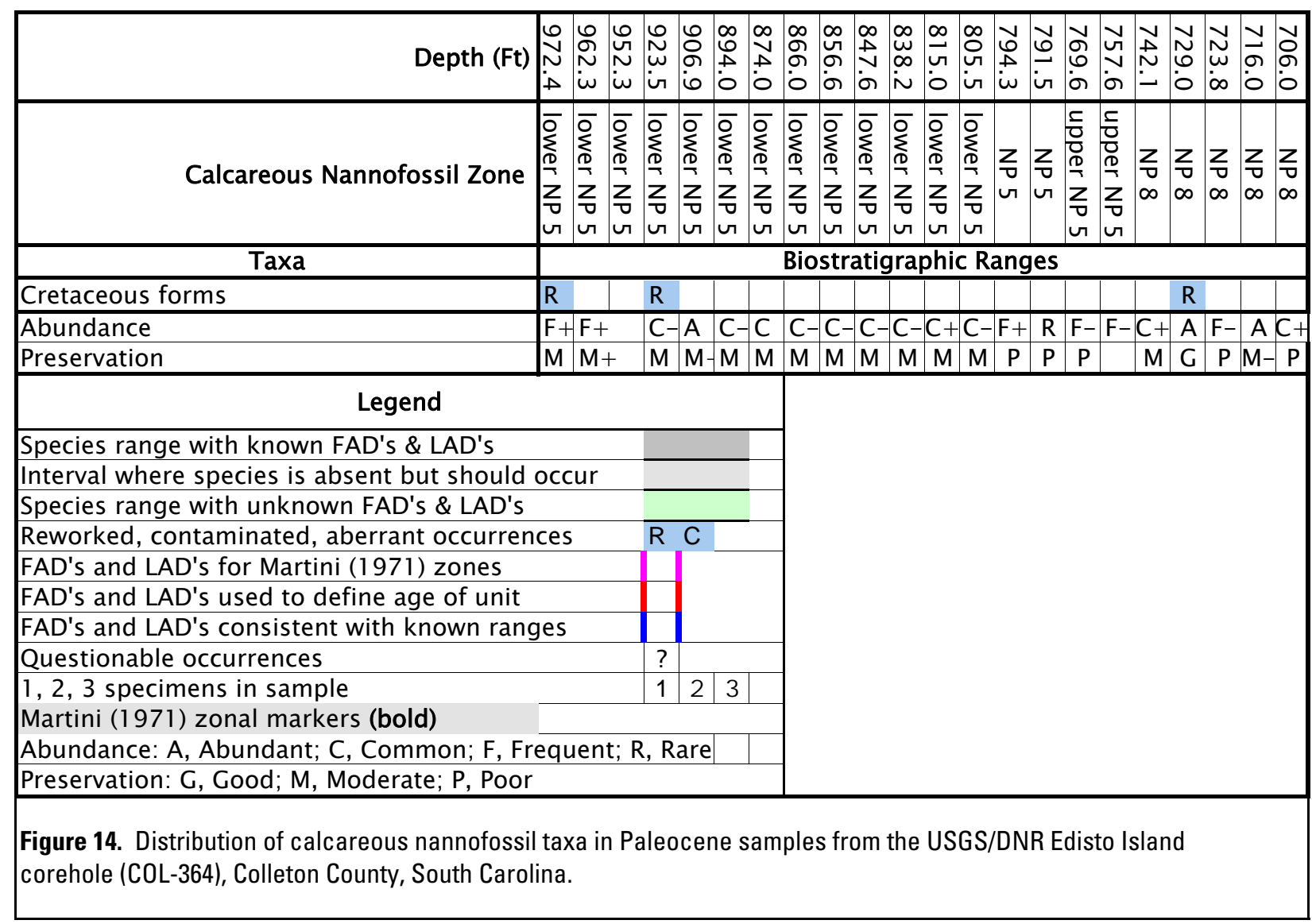




\begin{tabular}{|c|c|c|c|c|c|}
\hline Depth in $\mathrm{ft}$ & 972.1-972.4 & 901.0-901.3 & $823.0-823.3$ & 729.0-729.4 & 719.1-719.4 \\
\hline Sample & $\mathrm{R} 6122 \mathrm{AL}$ & R6122AK & $\mathrm{R} 6122 \mathrm{AJ}$ & \begin{tabular}{|l|}
$\mathrm{R} 6122 \mathrm{Al}$ \\
\end{tabular} & $\mathrm{R} 6122 \mathrm{AH}$ \\
\hline $\begin{array}{r}\text { Age or NP zone } \\
\text { equivalent }\end{array}$ & $\begin{array}{c}\text { Very sparse } \\
\text { pollen }\end{array}$ & $\begin{array}{l}\text { Apparently } \\
\text { NP5 }\end{array}$ & $\begin{array}{l}\text { Paleocene, } \\
\text { older than } \\
\text { mid-NP5? }\end{array}$ & $\begin{array}{c}\text { Paleocene, } \\
\text { NP5 or } \\
\text { younger }\end{array}$ & $\begin{array}{c}\text { Paleocene, } \\
\text { NP8 or } \\
\text { younger }\end{array}$ \\
\hline \multicolumn{6}{|l|}{ Taxa } \\
\hline Aesculiidites circumstriatus & . & . & . & $\mathrm{X}$ & . \\
\hline Carya $<29 \mu \mathrm{m}$ range base $=$ mid-NP5 & . & . & . & $\mathrm{X}$ & $\mathrm{X}$ \\
\hline Choanopollenites sp. cf. C. consanguineus & . & $\mathrm{X}$ & $\mathrm{X}$ & . & . \\
\hline Choanopollenites conspicuus Range top = NP5 & . & $\mathrm{X}$ & . & . & . \\
\hline Favitricolporites baculoferus & . & . & $\mathrm{X}$ & $\mathrm{X}$ & $\mathrm{X}$ \\
\hline Momipites actinus & . & . & . & . & $\mathrm{X}$ \\
\hline Momipites dilatus Range top $=$ NP8 & . & $\mathrm{X}$ & . & . & . \\
\hline Momipites strictus & . & . & . & . & $\mathrm{X}$ \\
\hline Nudopollis terminalis & . & . & $\mathrm{X}$ & . & $\mathrm{X}$ \\
\hline Nudopollis thiergartii & . & . & . & $\mathrm{x}$ & . \\
\hline Platycarya sp. A NP8 to lower Eocene & . & . & . & . & $\mathrm{X}$ \\
\hline Plicatopollis triorbicularis type & . & . & . & $\mathrm{X}$ & $\mathrm{X}$ \\
\hline Plicatopollis triradiatus & . & . & . & $\mathrm{X}$ & $\mathrm{X}$ \\
\hline Pseudoplicapollis limitatus & . & . & $\mathrm{X}$ & $\mathrm{X}$ & . \\
\hline Sparganiaceaepollenites sp. & . & . & . & $\mathrm{X}$ & . \\
\hline Spinaepollis spinosus Range base = approx. NP 4-5 bound. & . & $\mathrm{X}$ & . & $\mathrm{X}$ & . \\
\hline Thomsonipollis magnificus & . & . & $\mathrm{X}$ & $\mathrm{X}$ & $\mathrm{X}$ \\
\hline Trudopollis spp., including T. plenus & . & $\mathrm{X}$ & $\mathrm{X}$ &. & $\mathrm{X}$ \\
\hline \multicolumn{6}{|c|}{$\begin{array}{l}\text { Figure 15. Distribution of pollen taxa in Paleocene samples from the USGS/DNR Edisto Island corehole (COL-364), } \\
\text { Colleton County, South Carolina. }\end{array}$} \\
\hline
\end{tabular}




\begin{tabular}{|c|c|c|c|c|c|c|c|c|c|c|c|c|c|c|c|c|c|c|c|c|}
\hline & Depth $(F t)^{\star}$ & טै & $\begin{array}{l}1 \\
\infty \\
0\end{array}$ & సे & जै & $\underset{n}{\stackrel{H}{n}}$ & $\begin{array}{c}W \\
\omega \\
0\end{array}$ & N & రి & $\begin{array}{l}S \\
N \\
N\end{array}$ & $\begin{array}{l}9 \\
\omega \\
\omega\end{array}$ & $\begin{array}{l}\mathscr{S} \\
\mathbb{\omega} \\
\end{array}$ & N & 8 & \begin{tabular}{|l|}
$\pi$ \\
$\infty$ \\
\hdashline
\end{tabular} & $\begin{array}{l}c \\
\omega \\
\omega\end{array}$ & 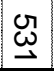 & $\begin{array}{l}N \\
N \\
\text { N }\end{array}$ & ज्ञ & जि \\
\hline & Sample & 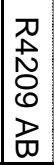 & $\begin{array}{l}D \\
\mathbb{N} \\
0 \\
0 \\
D \\
D\end{array}$ & $\begin{array}{l}D \\
0 \\
N \\
0 \\
0 \\
D \\
0\end{array}$ & $\begin{array}{l}D \\
\mathbb{1} \\
\mathbb{O} \\
0 \\
D \\
\text { 而 }\end{array}$ & $\begin{array}{l}0 \\
0 \\
\stackrel{1}{0} \\
0\end{array}$ & $\begin{array}{l}D \\
\mathbb{N} \\
0 \\
0 \\
D \\
\pi\end{array}$ & $\begin{array}{l}D \\
\mathbb{N} \\
\mathbb{N} \\
0 \\
D \\
D \\
B\end{array}$ & 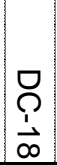 & $\begin{array}{l}D \\
\mathbb{N} \\
0 \\
0 \\
D \\
己\end{array}$ & $\begin{array}{l}0 \\
0 \\
\stackrel{\varphi}{v}\end{array}$ & $\begin{array}{l}D \\
\text { D } \\
\text { O } \\
0 \\
\text { D }\end{array}$ & $\begin{array}{l}D \\
\mathbb{D} \\
\mathrm{N} \\
0 \\
0 \\
\mathbb{D}\end{array}$ & 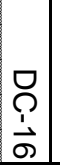 & $\begin{array}{l}20 \\
0 \\
0 \\
0 \\
I \\
I\end{array}$ & $\begin{array}{l} \\
\nabla \\
\cap \\
\dot{1} \\
\mathrm{v}\end{array}$ & $\begin{array}{l}\square \\
0 \\
1 \\
\dot{1} \\
\end{array}$ & $\begin{array}{l}\square \\
\bigcap \\
\dot{\omega} \\
\omega\end{array}$ & $\begin{array}{l}0 \\
n \\
\stackrel{n}{n} \\
\end{array}$ & $\begin{array}{l}\text { ? } \\
\stackrel{⿱ ⺊ 口}{\ominus}\end{array}$ \\
\hline Taxa & & & & & & & & & & & & & & & & & & & & \\
\hline Achomosphaera alcicornu (Eisenack 1954) Davey \& Williams 1966 & & . & . & . & . & . & . & . & . & . & . & . & . & . & . & . & . & . & $\mathrm{X}$ & \\
\hline Amphorosphaeridium ? multispinosum (Davey \& Williams 1966) Sarieant 1981 & & . &. &. &. &. & . &. &. & & . &. &. & . & $\mathrm{X}$ &. & . &. &. & \\
\hline Andalusiella sp. aff. A. polymorpha of Edwards (1980) & & . & . & . & . & . & . & . & . & . & . & . & . & $\mathrm{X}$ & . & . & . & . & . & \\
\hline Andalusiella Riegel 1974 spp. & & . &. &. & $\mathrm{X}$ & . & . & . & . & . & . & . & . &. &. &. &. & . & . & \\
\hline ?Andalusiella rhombohedra of Edwards and others (1984) & & . & $X$ & $\mathrm{X}$ & . & . & . & . & $\mathrm{X}$ & $\mathrm{X}$ & . & . & . & . & $\mathrm{X}$ & $\mathrm{X}$ & $\mathrm{X}$ & . & . & \\
\hline Apectodinium homomorphum (Defl. \& Cook. 1955) Lentin \& Williams 1977 & & . & . & . & . & . & . & . & . & . & . & . & . & . & . & . & . & . & . & $\mathrm{X}$ \\
\hline Areoligera volata Drugg 1967 & & $\mathrm{X}$ & . & $\mathrm{X}$ & $\mathrm{X}$ & . & $\mathrm{X}$ & $\mathrm{X}$ & . & $\mathrm{X}$ &. & . & $\mathrm{cf}$ & . & . & . & . &. & . & \\
\hline ?Canningia Cookson \& Eisenack 1960 sp. & & . & . & $\mathrm{X}$ & $x$ & . & $\mathrm{X}$ & $x$ & . & & . & . & $x$ & . & . & . & . & . & . & \\
\hline cf. Catillopsis Drugg 1970 & & . & $x$ & $x$ & . & . & . & $\mathrm{x}$ & . & . & . & . & . & . & . & . & . & . & . & . \\
\hline Cordosphaeridium fibrospinosum Davey \& Williams 1966 & & . &. & $\mathrm{X}$ & $\mathrm{X}$ & . & . & . & . & $\mathrm{X}$ & . & . & $\mathrm{X}$ & . & . & . & . & . & . & . \\
\hline Cordosphaeridium gracile (Eisenack 1954) Davey \& Williams 1966 & & . & . & . & . & . & . & . & . & . & . & . & $\mathrm{X}$ & . & . & . & . & . & . & $\mathrm{X}$ \\
\hline Cordosphaeridium inodes (Klumpp 1953) Eisenack 1963 & & $\mathrm{x}$ & $x$ & $\mathrm{x}$ & . & . & $\mathrm{X}$ & $\mathrm{X}$ & . & . & . & . & . & . & . & . & . & . & . & \\
\hline Cordosphaeridium Eisenack 1963 spp. & & . & . & . & . & . & . & . & . & $\mathrm{X}$ & . & . & $\mathrm{X}$ & . & $\mathrm{X}$ & . & . & . & . & \\
\hline Damassadinium californicum (Drugg 1967) Fensome et al. 1993 & & $\mathrm{X}$ & $?$ & . & . & . & $\mathrm{X}$ & $\mathrm{X}$ & . & $\mathrm{X}$ & . & . & $\mathrm{X}$ & . & $\mathrm{X}$ & . & . & $?$ & $\mathrm{X}$ & \\
\hline Deflandrea delineata Cookson \& Eisenack 1965 & & . & . & . & . & . & . & . & . & . & . & . & . & . & $\mathrm{X}$ & $\mathrm{X}$ & $x$ & . & $\mathrm{X}$ & $x$ \\
\hline Deflandrea cf. D. diebelii Alberti of Drugg (1967) & & $\mathrm{X}$ & $x$ & $\mathrm{x}$ & $x$ & . & $\mathrm{x}$ & . & . & $\mathrm{X}$ & $x$ & . & . & . & . & . & . & . & . & . \\
\hline Deflandrea n. sp. aff. D. truncata Eisenack 1938 & & $\mathrm{X}$ & $\mathrm{X}$ & $\mathrm{X}$ & cf & . & $\mathrm{X}$ & $\mathrm{X}$ & . & $\mathrm{X}$ & . & . & . & . & . & . & . & . & . & \\
\hline Diphyes colligerum (Deflandre \& Cookson 1955) Cookson 1965 & & $\mathrm{X}$ & $\mathrm{X}$ & $\mathrm{x}$ & . & . & $\mathrm{X}$ & $\mathrm{X}$ & . & . & . & . & $\mathrm{X}$ & . & . & . & . & . & . & \\
\hline Disphaerogena carposphaeropsis Wetzel 1933 & & . & $\mathrm{X}$ & . & . & . & . & . & . & . & . & . & . & . & . & . & . & . & . & \\
\hline Exochosphaeridium Davey et al. 1966 spp. & & $\mathrm{X}$ & $x$ & $\mathrm{X}$ & $x$ & $\mathrm{X}$ & $\mathrm{X}$ & $x$ & . & $\mathrm{x}$ & $x$ & . & cf & $x$ & . & . & . & . & . & \\
\hline Fibradinium annetorpense Morgenroth 1968 & &. & $\mathrm{X}$ & . & . & . & . & . & . & . & . & . & $\mathrm{X}$ & . & . & . & . & . & . & \\
\hline Fibrocysta lappacea (Drugg 1970) Stover \& Evitt 1978 & & . & . & . & $?$ & . & . & $x$ & . & . & . & . & . & . & . & . & . & . & . & \\
\hline Gonyaulacacysta group & & $\mathrm{X}$ & . & . & . & . & . & . & . & . & . & . & . & . & . & . & . & . & . & \\
\hline Hafniasphaera septata (Cookson \& Eisenack 1967) Hansen 1977 & & . & $?$ & $\mathrm{X}$ & $\mathrm{X}$ & . & . & . & . & . & . & . & $\mathrm{X}$ & . & . & . & . & . & . & \\
\hline Hafniasphaera Hansen 1977 sp. & & $x$ & $x$ & $\mathrm{X}$ & . &. &. &. &. & . &. & . & . &. &. &. &. & . &. & \\
\hline Hystrichokolpoma Deflandre 1935 spp. & & . & $x$ & $x$ & . & . & . & $x$ & . & . & . & . & . & . & . & . & . & . & . & \\
\hline Hystrichosphaeridium tubiferum (Ehrenberg 1838) Deflandre 1937 & & . & $x$ & $\mathrm{X}$ & . & . & $\mathrm{X}$ & $x$ & . & . & . & . & . & . & . & . & . & . & . & \\
\hline Impagidinium Stover \& Evitt 1978 spp. (and transitional forms) & & . & $\mathrm{X}$ & $\mathrm{X}$ & . & . & $\mathrm{X}$ & $\mathrm{X}$ & . & $\mathrm{X}$ & . & . & $\mathrm{X}$ & . & . & . & . & . & . & \\
\hline Ifecysta Jan du Chêne \& Adediran 1985 sp. & & $\mathrm{X}$ & $\mathrm{X}$ & . & . & . & . &. & . & & . & . &. & . & . & . & . & . &. & \\
\hline
\end{tabular}




\begin{tabular}{|c|c|c|c|c|c|c|c|c|c|c|c|c|c|c|c|c|c|c|c|c|}
\hline & Depth $(\mathrm{Ft})^{\star}$ & ปै & $\begin{array}{l}1 \\
\infty \\
0\end{array}$ & హె & जे & $\stackrel{B}{P}$ & $\begin{array}{c}\tilde{\omega} \\
\mathscr{\omega}\end{array}$ & $\begin{array}{l}N \\
\mathcal{O}\end{array}$ & ठั & $\begin{array}{l}\text { N } \\
\text { N }\end{array}$ & $\begin{array}{l}\stackrel{8}{\omega} \\
\end{array}$ & 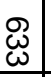 & 尺્) & 8 & 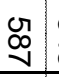 & $\begin{array}{l}\sigma \\
\omega \\
\omega\end{array}$ & 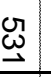 & & & ज \\
\hline \multicolumn{21}{|c|}{ 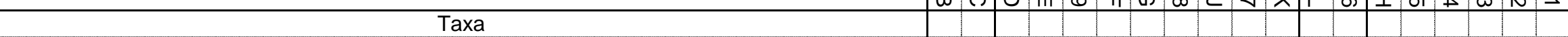 } \\
\hline Kallosphaeridium de Coninck1969? sp. & & . &. & & & . & . & . & . & . &. & . & $\mathrm{X}$ & & . &. & . & . & & . \\
\hline Operculodinium centrocarpum (Deflandre \& Cookson 1955) Wall 1967 & & . & $x$ & $x$ & $\mathrm{x}$ & &. & $\mathrm{x}$ & & $\mathrm{x}$ & . & . & . & & $\mathrm{X}$ & . & . & & & \\
\hline Palaeoperidinium pyrophorum (Ehrenberg 1838) Sarjeant 1967 & & . & $x$ & $\mathrm{x}$ & $x$ &. &. &. &. & $\cdot$ & . & . & . & & $\mathrm{x}$ & . & . &. & . & . \\
\hline Phelodinium magnificum (Stanley 1965) Stover \& Evitt 1978 & & . & $x$ & $\mathrm{x}$ &. & . &. & . & $\mathrm{x}$ & $x$ & $x$ & . & . & &. & . & . &. & & . \\
\hline Phelodinium sp. of Edwards (1989) & & . &. & . & & . & . & . & & & . & . & $\mathrm{X}$ & & $\mathrm{X}$ & . & . & . & $\mathrm{x}$ & \\
\hline Phelodinium Stover \& Evitt 1978 sp. & & $x$ &. &. & . & $\mathrm{X}$ & $x$ & $\mathrm{x}$ & . & . &. & . & . & & . & . & . & . & & \\
\hline Senoniasphaera inornata (Drugg 1970) Stover \& Evitt 1978 & & . & $x$ &. & . &. &. & . & . & $\mathrm{x}$ &. & . &. & & . & . & . & . & . & . \\
\hline Spinidinium pulchrum (Benson 1976) Lentin \& Williams 1977 & & $\mathrm{x}$ & $\mathrm{x}$ & $\mathrm{X}$ & $\mathrm{x}$ & $\mathrm{X}$ & $x$ & $\mathrm{X}$ & $\mathrm{X}$ & $\mathrm{X}$ &. & . & cf & & . & . & . & & & . \\
\hline Tenua sp. cf. T. formosa of Kurita and McIntyre (1995) & & . &. & $\mathrm{x}$ & cf & . & cf & . & & $\mathrm{X}$ &. & $\mathrm{x}$ &. & & . & . & . & . & & \\
\hline Trithyrodinium Drugg $1967 \mathrm{sp.}$ & & $?$ &. & & & . &. & . & & & . &. &. & & & . & . & . & & \\
\hline Tubiosphaera filosa (Wilson 1967) Archangelsky 1969 & &. &. & $\cdot$ & . & . &. & . & . & . &. &. & $\mathrm{x}$ & & . & . & . &. & . & . \\
\hline Turbiosphaera sp. aff. T. magnifica Eaton of Edwards (1989) & & . &. &. & & . & . & $!$ & & &. & . & . & & . & . & . & . & $x$ & \\
\hline Veryhachium -group acritarch & & . & $x$ & . &. & . &. & . & . & . &. & . & . & & . & . & . & . & 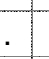 & \\
\hline miscellaneous areoligeracean forms & & $\mathrm{X}$ & $x$ & $x$ & $x$ & . &. & $x$ & & &. & $\cdot$ & $\mathrm{X}$ & & $\mathrm{X}$ & . & . &. & & \\
\hline miscellaneous cladopyxiaceaen forms & & $\mathrm{x}$ & $x$ & & & . &. & . & & &. & . &. & & & . & . & & & \\
\hline small peridiniacean forms & & . & $\mathrm{x}$ & $x$ & $\mathrm{x}$ & $\mathrm{x}$ & $x$ & $\mathrm{x}$ & $\mathrm{x}$ & $\mathrm{x}$ & $x$ & $\mathrm{x}$ & $\mathrm{X}$ & $\mathrm{X}$ & $\mathrm{x}$ & . & . & & $x$ & \\
\hline \multicolumn{21}{|l|}{ *Depths recorded at the time of sampling. To convert to log depths, add $5 \mathrm{ft}$. } \\
\hline $\mathrm{cf}=$ similar to this taxon. & & & & & & & & & & & & & & & & & & & & \\
\hline & & & & & & & & & & & & & & & & & & & & \\
\hline
\end{tabular}




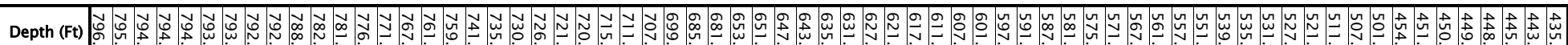

Calcareous Nannofossil Zone

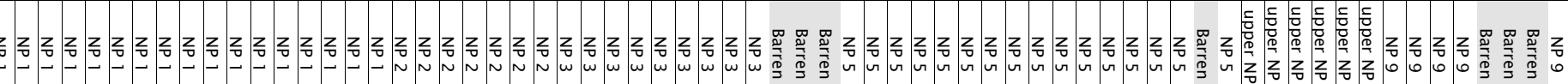

\begin{tabular}{l} 
Taxa \\
\hline Biantholithus sparsus NP 1-9 \\
Biscutum spp. NP 1-12? \\
Blackites spp. \\
Braarudosphaera bigelowii Cret-Recent
\end{tabular} Chiasmolithus bidens NP 5-16 Chiasmolthus consuetus s.l. NP

Chiasmolithus spp.

Coccolithus eopelagicus

Coccolithus pelagicus Criciplacolithus asymmetricus $\mathrm{NP}$ 1-5 Cruciph colithus interds N NP 2?-5? Cruciplacolithus intermedius NP

Cruciplacolithus tenuis NP 2-9

Cruciplacolithus spp.

Cyclagelosphaera reinhardtii NP 1-5

Cyclagelosphaera spp.

Cyclococcolithus robustus

Cyclococcolithus sp.

Discoaster multiradiatus NP 9-11

Discoaster spp.

Ellipsolithus macellus NP 4-12

Ellipsolithus sp.

Ericsonia subpertusa NP 1-12?

Fasciculithus involutus NP 5-10

Fasciculithus janil NP 4-5

Fasciculithus tympaniformis NP 5-10

Fasciculithus ulii NP 5

Fasciculithus spp.

Coniolithus fluckigeri Cret-NP 25?

Helicosphaera compacta NP 16-24

Helicosphaera spp.
Helithus cantabriae NP 5-8

Heliolithus spp. NP 5-9

Hornibrookina spp.

Lanternithus duocavus NP 1-5?

anternithus sp

Whas apertus NP 3-10?

Micrantholithus as NP 1-21

Micrantholithus pinguis

Micrantholithus spp.

Neochiastozygus concinnus NP 2?-11

Neococcolithes sp. aff. N. protenus

Neococcolithes spp.

Neacrepidolithus sp.
Placozygus sigmoides NP 1-10

Praeprinsius sp.

Reticulofenestra floridain NP 13Reticulofenestra hillae

Reticulofenestra placomorpha

Reticulofenestra umblicus NP 16-22

Reticulofenestra spp.

Thoracosphaera spp.

Toweius eminens tovae NP 5-9

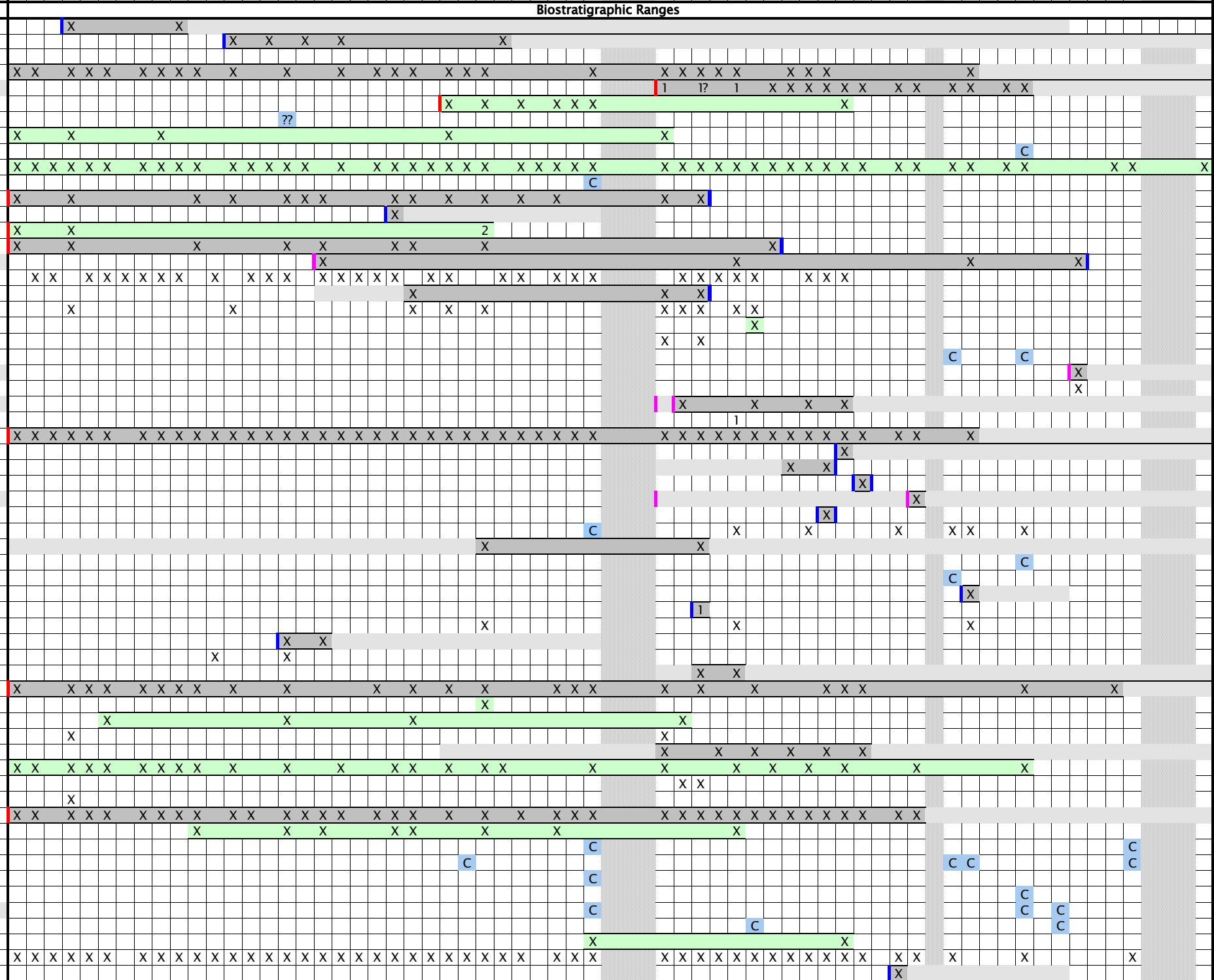




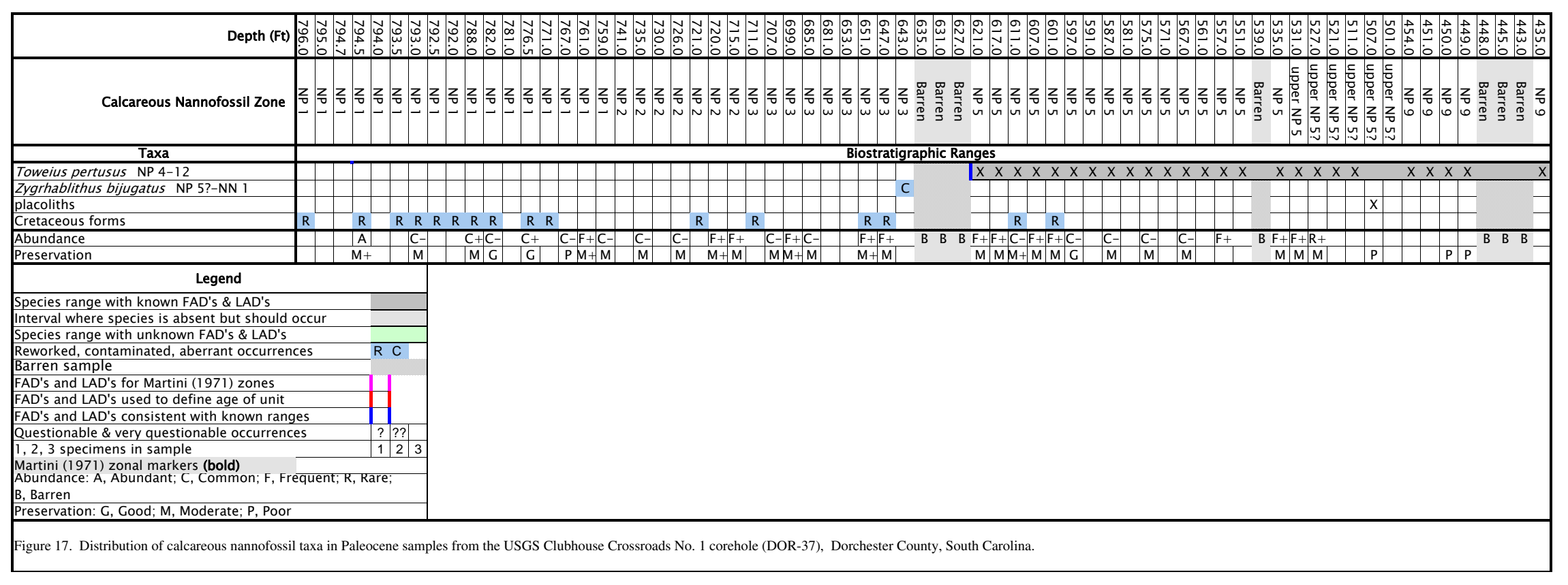




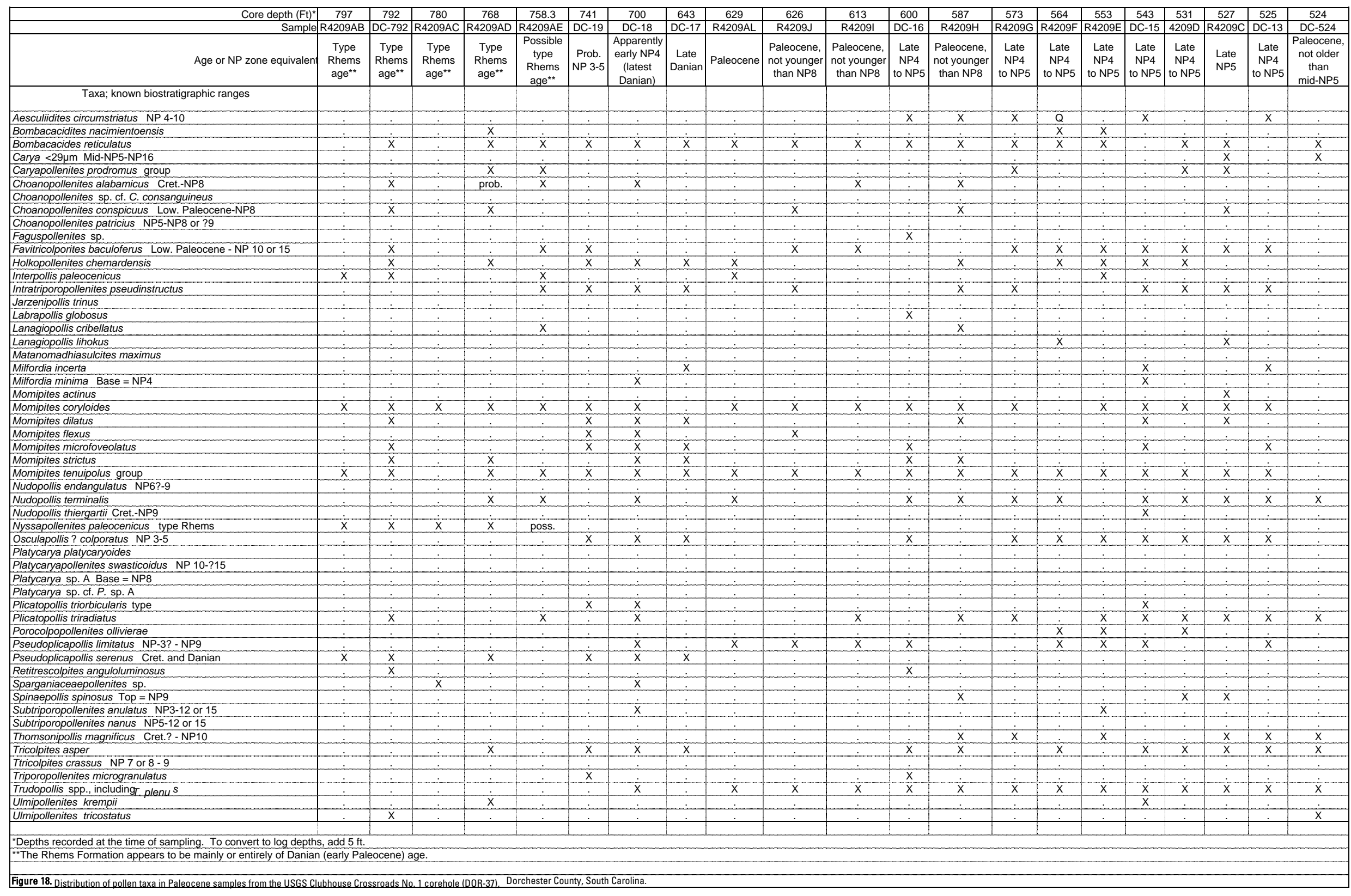




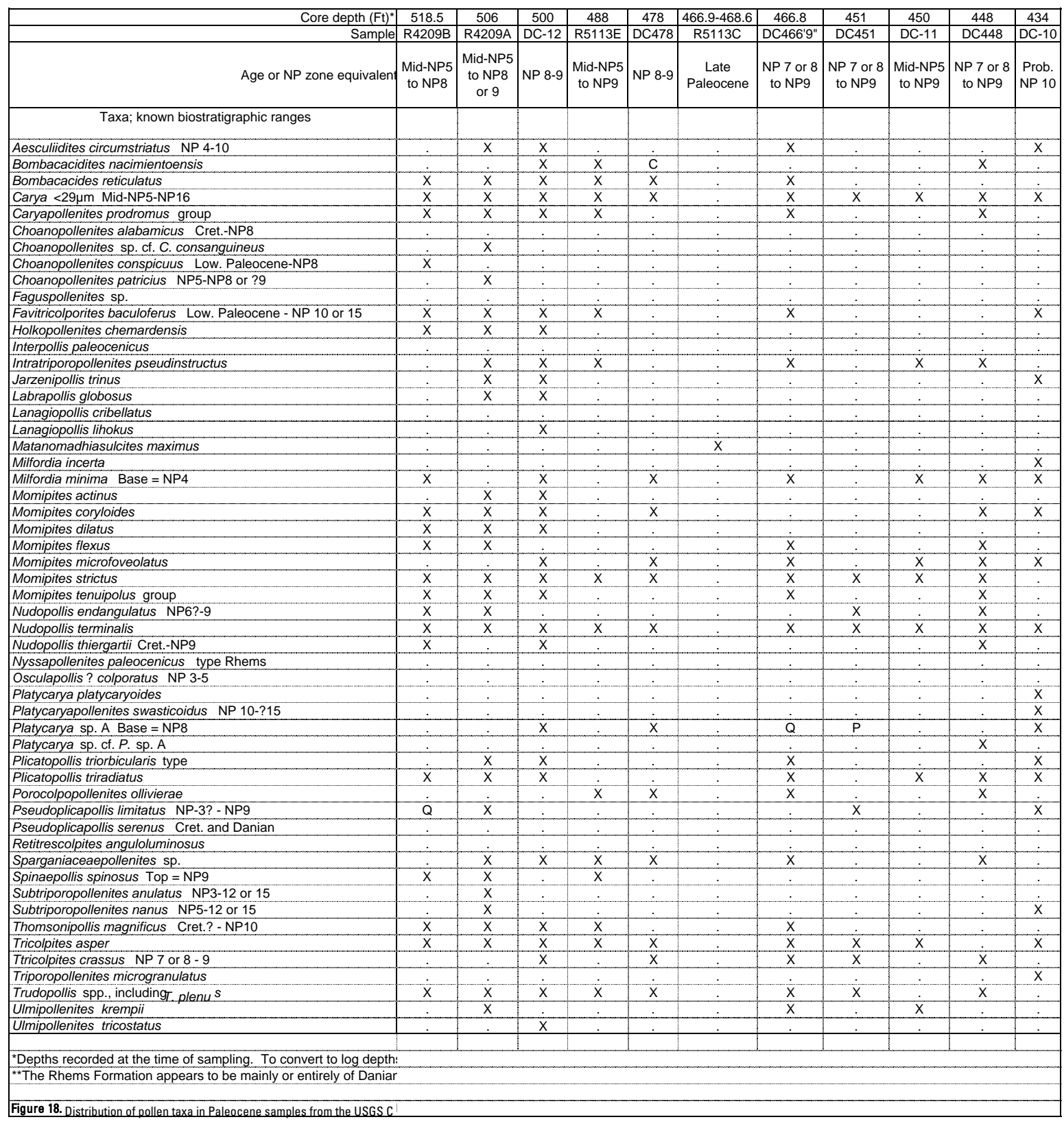




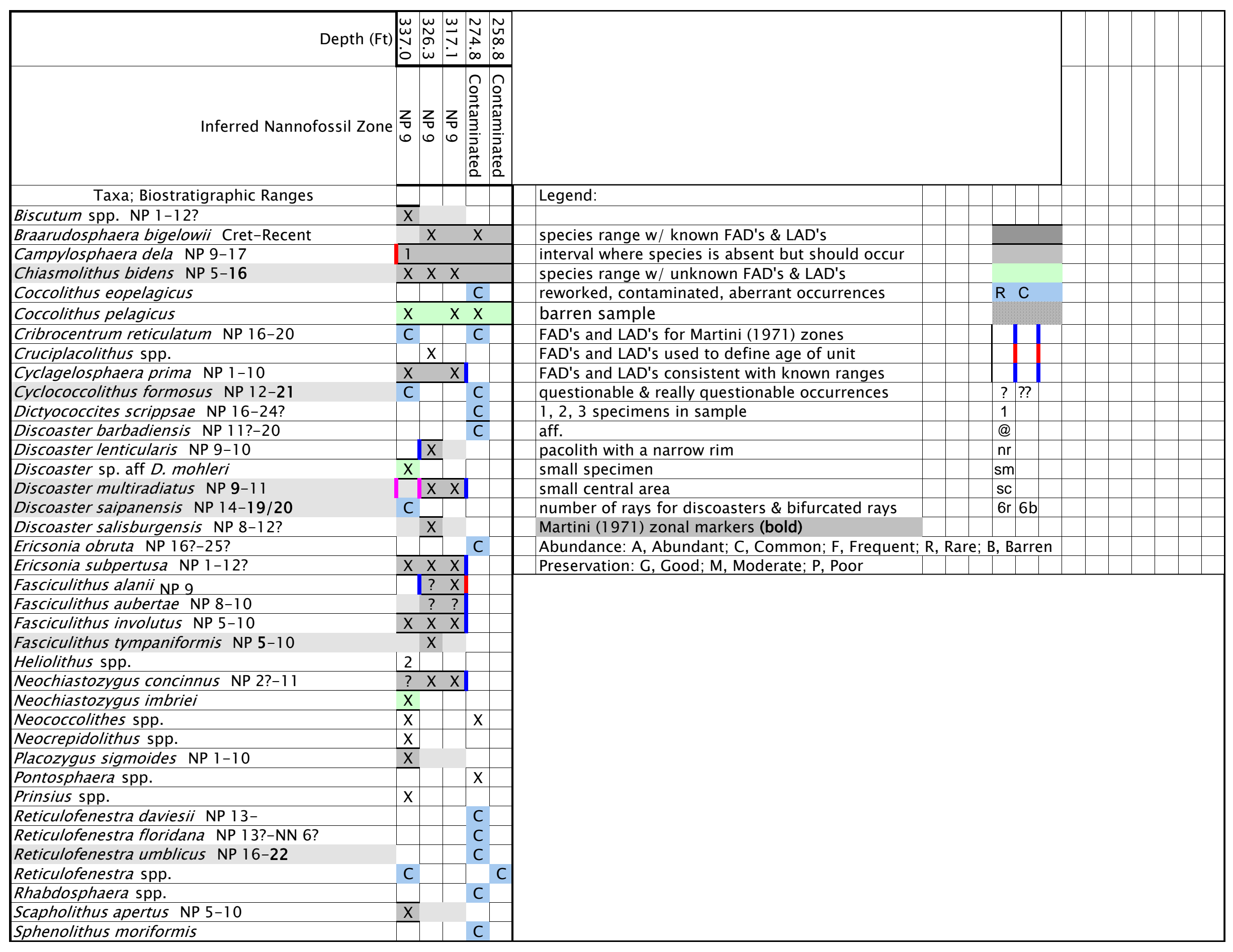




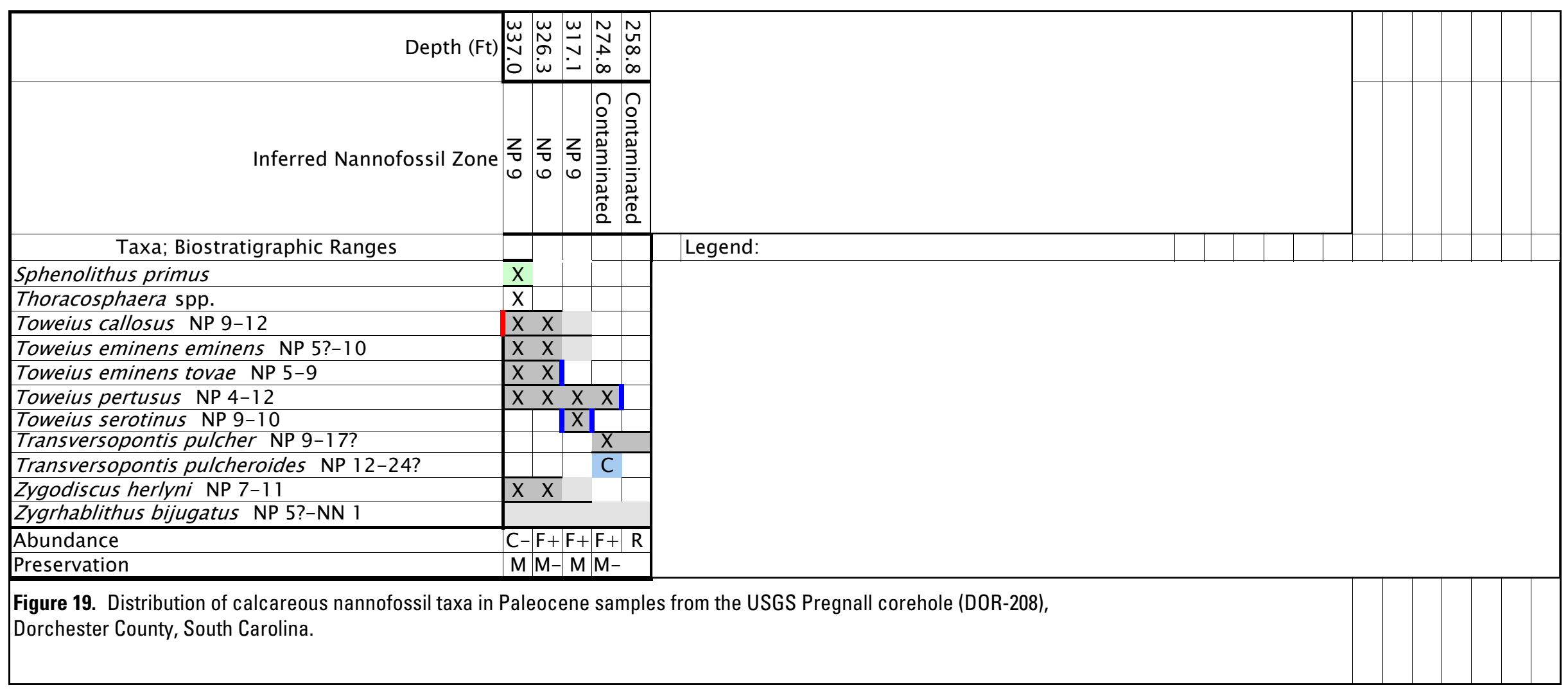




\begin{tabular}{|c|c|c|c|}
\hline Depth $(\mathrm{Ft})$ & 510 & 376 & 300 \\
\hline Sample & R3001 AC & R3001 AD & R3001 AE \\
\hline \multicolumn{4}{|l|}{ Taxa; Stratigraphic Ranges } \\
\hline Adnatosphaeridium robustum (Morgenroth 1966) de Coninck 1975 & . & . & $\bar{X}$ \\
\hline Amphorosphaeridium? multispinosum (Davey \& Williams 1966) Sarjeant 198 & . & . & $\mathrm{X}$ \\
\hline Apectodinium homomorphum (Defl. \& Cook. 1955) Lentin \& Williams 1977 & . & . & $\mathrm{X}$ \\
\hline Cerodinium diebelii (Alberti 1959) Lentin \& Williams 1987 & $\mathrm{X}$ & . & . \\
\hline Cordosphaeridium fibrospinosum Davey \& Williams 1966 & $\mathrm{x}$ & . & . \\
\hline Cordosphaeridium gracile (Eisenack 1954) Davey \& Williams 1966 & $\mathrm{X}$ & . & $\mathrm{X}$ \\
\hline Damassadinium californicum (Drugg 1967) Fensome et al. 1993 & $\mathrm{X}$ & . & . \\
\hline Deflandrea phosphoritica Eisenack 1938 & . & . & $\mathrm{X}$ \\
\hline Fibradinium annetorpense Morgenroth 1968 & $\mathrm{X}$ & . & . \\
\hline Hafniasphaera septata (Cookson \& Eisenack 1967) Hansen 1977 & $\mathrm{X}$ & . & . \\
\hline Heteraulacacysta Drugg \& Loeblich 1967 sp. & . & . & $\mathrm{X}$ \\
\hline Hystrichosphaeridium tubiferum (Ehrenberg 1838) Deflandre 1937 & . & . & $\mathrm{X}$ \\
\hline Impagidinium Stover \& Evitt 1978 sp. & . & . & $\mathrm{X}$ \\
\hline Isabelidinium bakeri (Defl. \& Cook. 1955) Lentin \& Williams 1977 & $\mathrm{X}$ & . & . \\
\hline Kallosphaeridium brevibarbatum de Coninck 1969 & . & . & $\mathrm{X}$ \\
\hline Lejeunecysta Artzner \& Dörhöfer 1978 sp. & $\mathrm{X}$ & . & . \\
\hline Palaeocystodinium golzowense Alberti 1961 & $\mathrm{X}$ & . & . \\
\hline Palaeocystodinium Alberti 1961 sp. & $\mathrm{X}$ & . & . \\
\hline Palaeoperidinium pyrophorum (Ehrenberg 1838) Sarjeant 1967 & $\mathrm{X}$ & . & . \\
\hline Phelodinium sp. of Edwards (1989) & $\mathrm{X}$ & . & $\mathrm{X}$ \\
\hline Senegalinium microgranulatum (Stanley 1965) Stover \& Evitt 1978 & $\mathrm{X}$ & . & . \\
\hline Senegalinium obscurum (Drugg 1967) Stover \& Evitt 1978 & $\mathrm{X}$ & . & . \\
\hline Senegalinium? dilwynense (Cookson \& Eisenack 1965) Stover \& Evitt 1978 & . & . & $\mathrm{X}$ \\
\hline Spiniferites Mantell 1850 spp. & $\mathrm{X}$ & . & $\mathrm{X}$ \\
\hline Thalassiphora pelagica (Eisenack 1954) Eisenack \& Gocht 1960 & $?$ & . & . \\
\hline Turbiosphaera sp. aff. T. magnifica Eaton of Edwards (1989) & . & . & $?$ \\
\hline miscellaneous areoligeracean forms & $\mathrm{x}$ & . & . \\
\hline small peridiniacean forms & $\mathrm{X}$ & . & . \\
\hline dinoflagellate fragments, indeterminate & . & $\mathrm{X}$ & . \\
\hline Pediastrum (freshwater alga) & . & $\mathrm{X}$ & $\mathrm{X}$ \\
\hline $\begin{array}{l}\text { Figure 20. Distribution of dinocyst taxa in Paleocene samples from the USGS St. Geo } \\
\text { (DOR-211), Dorchester County, South Carolina. } X=\text { present; ? = questionable. }\end{array}$ & rge corehole & & \\
\hline
\end{tabular}




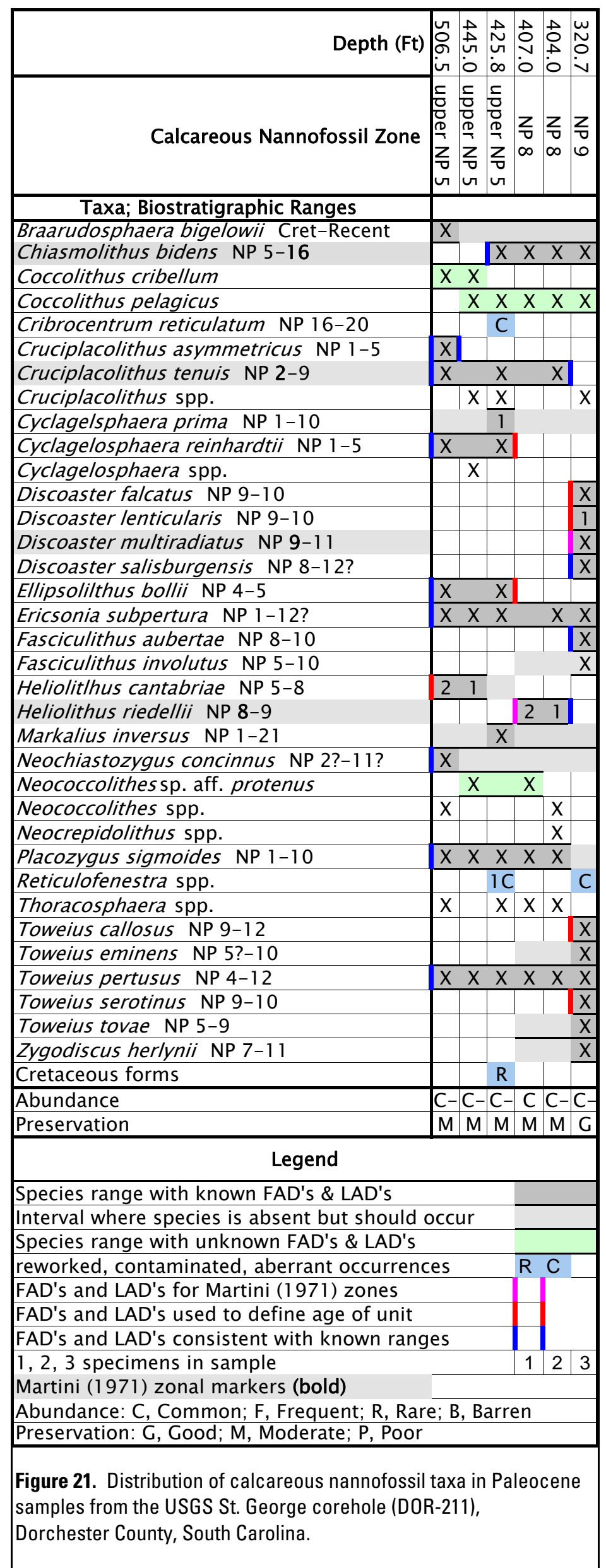




\begin{tabular}{|c|c|c|c|c|c|c|c|c|c|c|c|c|c|}
\hline Depth (Ft) & & & & & & & $\begin{array}{l}\infty \\
\infty \\
\sim\end{array}$ & & & & & & $\tilde{o}_{0}^{v}$ \\
\hline Calcareous Nannofossil Zone & $\frac{z}{v}$ & & 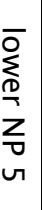 & & & 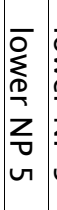 & $\begin{array}{l}\bar{o} \\
\sum_{0} \\
\underline{0} \\
z \\
u \\
v\end{array}$ & & & & $\begin{array}{l}\frac{c}{0} \\
\frac{0}{0} \\
\mathbb{D} \\
Z \\
Z \\
v \\
v\end{array}$ & & 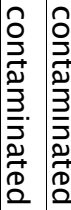 \\
\hline Taxa & & & & & stra & atig & grap & phi & ic $R_{i}$ & ang & & & \\
\hline Blackites spp. & & & & & & & & & & & & & $\mathrm{X}$ \\
\hline Braarudosphaera bigelowii Cret-Recent & $\mathrm{X}$ & & & $\mathrm{X}$ & & & $\mathrm{X}$ & & & & & & $\mathrm{x}$ \\
\hline Cepekiella lumina NP 11-24? & & & & & & & & & & & & & $\mathrm{C}$ \\
\hline Chiasmolithus bidens NP 5-16 & & & $\bar{X}$ & $\mathrm{X}$ & $\mathrm{X}$ & & & $\mathrm{X}$ & $\mathrm{X}$ & $\mathrm{x}$ & & $\mathrm{x}$ & \\
\hline Chiasmolithus sp. aff. C. bidens NP 4 & & & & & & & $1 ?$ & & & & & & \\
\hline Chiasmolithus consuetus s.I. NP 3- & & & $\mathrm{X}$ & $\mathrm{X}$ & & & & & $\mathrm{X}$ & & & & \\
\hline Chiasmolithus grandis NP 14-17 & & & & & & & & & & & & & C \\
\hline Chiasmolithus spp. small unsplit & & & & & & & & & & & & $\mathrm{X}$ & \\
\hline Coccolithus pelagicus & & & $\mathrm{X}$ & $\mathrm{X}$ & $\mathrm{X}$ & & $\mathrm{X}$ & & $\mathrm{X}$ & $\mathrm{X}$ & $\mathrm{X}$ & $\mathrm{X}$ & $\mathrm{x})$ \\
\hline Cribrocentrum reticulatum NP 16-20 & & & & & & & & & & & & $\mathrm{C}$ & \\
\hline Cruciplacolithus asymmetricus NP 1-5 & $\mathrm{x}$ & & $\mathrm{X}$ & $\mathrm{X}$ & $\mathrm{X}$ & $\mathrm{x}$ & $\mathrm{X}$ & & & & & & \\
\hline Cruciplacolithus intermedius NP 1- & $\bar{X}$ & & & & & & & & & & & & \\
\hline Cruciplacolit/hus primus NP 1-5 & $\bar{x}$ & & & & & & & & & & $\bar{X}$ & & \\
\hline Cyclagelosphaera prima NP 1-10 & & & & & $\mathrm{X}$ & & & & $\mathrm{X}$ & & & & \\
\hline Cyclococcolithus formosus NP 12-21 & & & & & & & & & & & & C & C \\
\hline Daktylethra punctulata NP 15-17 & & & & & & & & & & & & & C \\
\hline Dictyococcites bisectus NP 16-NN 1 & & & & & & & & & & & & & $\mathrm{C}$ \\
\hline Dictyococcites scrippsae NP 16-24? & & & & & & & & & & & & C & C \\
\hline Discoaster barbadiensis NP $11 ?-20$ & & & & & & & & & & & & & C \\
\hline Discoaster mohleri NP 7-8? & & & & & & & & & & & C? & & \\
\hline Ellipsolithus bollii NP 4-5 & & & & $\bar{x}$ & $\mathrm{X}$ & & & & & & & & \\
\hline Ellipsolithus distichus NP 4-12 & & & & & & & & $\bar{x}$ & & & & & \\
\hline Ellipsolithus macellus NP 4-12 & & & & & & & & $\mathrm{x}$ & & & & & \\
\hline Ericsonia subpertusa NP 1-12? & $\mathrm{X}$ & & $\bar{x}$ & $\mathrm{X}$ & $\mathrm{X}$ & $\mathrm{X}$ & $\mathrm{X}$ & $\mathrm{X}$ & $\mathrm{X}$ & $\mathrm{X}$ & & $\mathrm{X}$ & \\
\hline Fasciculithus janii/billii NP 4-5 & & & & & & & & $\mathrm{X}$ & & & & & \\
\hline Fasciculithus involutus NP 5-10 & & & & & & & $\mathrm{X}$ & & & & & $\mathrm{X}$ & \\
\hline Fasciculithus tympaniformis NP 5-10 & & & & & & & & & $\mathrm{X}$ & & $\mathrm{X}$ & & \\
\hline Fasciculithus spp. & & & & & & & & $\mathrm{X}$ & & & $\mathrm{X}$ & & \\
\hline Goniolithus fluckigeri Cret-NP 25? & $\mathrm{X}$ & & & & & & & & & & & & \\
\hline Helicosphaera compacta NP 16-24 & & & & & & & & & & & & & C \\
\hline Heliolithus cantabriae NP 5-8 & & & & & & & & & & & & $\mathrm{X}$ & \\
\hline Heliolithus kleinpellii NP 6-8 & & & & & & & & & & & & $\mathrm{X}$ & \\
\hline Hornibrookina arca NP 3-10 & & & & & & & & & & & & & 1 \\
\hline Markalius inversus NP 1-21 & $\mathrm{X}$ & & & & & & & & & & & & $\bar{x}$ \\
\hline Neochiastozygus concinnus NP 2?-11 & & & $\mathrm{X}$ & & & & & & & & & & \\
\hline Neococcolithes sp. aff. N. protenus & $\mathrm{X}$ & & $\mathrm{X}$ & $\mathrm{X}$ & & & & $\mathrm{X}$ & $\mathrm{X}$ & & & & \\
\hline Neococcolithes spp. & & & $\mathrm{X}$ & & & & & & & & & $\mathrm{X}$ & \\
\hline Placozygus sigmoides NP 1-10 & $\mathrm{x}$ & & $\mathrm{X}$ & $\mathrm{X}$ & & & $\mathrm{X}$ & $\mathrm{X}$ & $\mathrm{x}$ & & & & \\
\hline Praeprinsius spp. & $\bar{X}$ & & & & & & & & & & & & \\
\hline Reticulofenestra floridana NP13?-NN 6? & & & & & & & & & & & & & \\
\hline Reticulofenestra umblicus NP 16-22 & & & & & & & & & & & & & C \\
\hline Reticulofenestra spp. & & & & & & & & & & & & C & \\
\hline Reticulofenestra spp. small & & & & & & & & & & & & & C \\
\hline Thoracosphaera spp. & $\mathrm{X}$ & & $\mathrm{X}$ & $\mathrm{X}$ & $\mathrm{X}$ & $\mathrm{X}$ & & $\mathrm{X}$ & $\mathrm{X}$ & & & & \\
\hline Toweius eminens eminens NP 5?-10 & & & & & & & & & $\mathrm{X}$ & & & $\mathrm{X}$ & \\
\hline Toweius eminens tovae NP 5-9 & & & & & & & & & $\bar{X}$ & & $\mathrm{X}$ & $\bar{X}$ & \\
\hline Toweius pertusus NP 4-12 & & & $\mathrm{X}$ & $\mathrm{X}$ & $\mathrm{X}$ & & $\mathrm{X}$ & $\mathrm{x}$ & $\mathrm{X}$ & $\mathrm{X}$ & $\mathrm{X}$ & $\mathrm{X}$ & \\
\hline Transversopontis pulcher NP 9-17? & & & & & & & & & & & & & C \\
\hline Transversopontis pulcheroides NP 12-24? & & & & & & & & & & & & & C \\
\hline Zygodiscus herlyni NP 7-11 & & & & & & & & & & & $\mathrm{X}$ & & \\
\hline Zygrhablithus bijugatus NP 5?-NN 1 & & & & & & & & & & & & & \\
\hline placoliths & & $\mathrm{X}$ & & & & & & & & & & & \\
\hline
\end{tabular}




\begin{tabular}{|c|c|c|c|c|c|c|c|c|c|}
\hline Depth (Ft) & 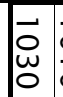 & $\begin{array}{ll}\overrightarrow{0} & \overrightarrow{8} \\
0 & 0 \\
\end{array}$ & $\begin{array}{lll}0 & 0 \\
0 & 0\end{array}$ & \begin{tabular}{l|l}
$w$ & $\infty$ \\
$w$ & $\infty$ \\
0 & $\sim$
\end{tabular} & \begin{tabular}{l|l}
$\infty$ & $\infty$ \\
0 & 0 \\
v & 0
\end{tabular} & $\begin{array}{l}\infty \\
0 \\
0\end{array}$ & $\infty$ & วั & wै \\
\hline Calcareous Nannofossil Zone & $\begin{array}{l}z \\
z \\
v \\
v\end{array}$ & 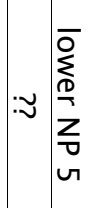 & 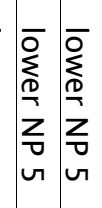 & 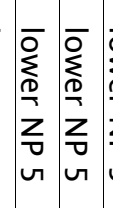 & 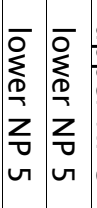 & 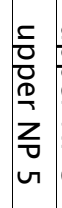 & 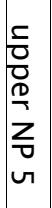 & $\begin{array}{l}z \\
\text { zo } \\
a\end{array}$ & 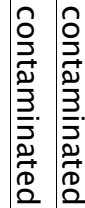 \\
\hline Taxa & \multicolumn{9}{|c|}{ Biostratigraphic Ranges } \\
\hline Cretaceous forms & $\mathrm{R}$ & & $\mathrm{R} R$ & $\mathrm{R}$ & & & & & \\
\hline Abundance & F- & $\mathrm{R} F+$ & $+\mathrm{F}+\mathrm{F}+$ & $+\mathrm{F}-\mathrm{F}+\mathrm{F}$ & $\mathrm{F}+\mathrm{F}+$ & $\mathrm{F}+$ & & $\mathrm{F}+$ & $\mathrm{A} F$ \\
\hline Preservation & $\mathrm{M}$ & $\mathrm{PM}+$ & $+\mathrm{MM}+$ & $+\mathrm{MM}+$ & $\mathrm{MM}+$ & $+\mathrm{M}$ & G & $\mathrm{P}$ & \begin{tabular}{l|l}
$\mathrm{G}$ & $\mathrm{P}$ \\
\end{tabular} \\
\hline \multicolumn{4}{|l|}{ Legend } & & & & & & \\
\hline \multicolumn{4}{|l|}{ Species range with known FAD's \& LAD's } & & & & & & \\
\hline \multicolumn{4}{|l|}{ Interval where species is absent but should occur } & & & & & & \\
\hline \multicolumn{4}{|l|}{ Species range with unknown FAD's \& LAD's } & & & & & & \\
\hline Reworked, contaminated, aberrant occurrences & & $\mathrm{R}$ & $\mathrm{C}$ & & & & & & \\
\hline \multicolumn{4}{|l|}{ FAD's and LAD's for Martini (1971) zones } & & & & & & \\
\hline \multicolumn{4}{|l|}{ FAD's and LAD's used to define age of unit } & & & & & & \\
\hline \multicolumn{4}{|l|}{ FAD's and LAD's consistent with known ranges } & & & & & & \\
\hline \multicolumn{2}{|l|}{ Questionable occurrences } & $?$ & & & & & & & \\
\hline \multicolumn{4}{|l|}{$1,2,3$ specimens in sample } & & & & & & \\
\hline \multicolumn{4}{|l|}{ Martini (1971) zonal markers (bold) } & & & & & & \\
\hline \multicolumn{4}{|c|}{ Abundance: C, Common; F, Frequent; R, Rare; B, Barren } & & & & & & \\
\hline \multicolumn{4}{|c|}{ Preservation: G, Good; M, Moderate; P, Poor } & & & & & & \\
\hline
\end{tabular}




\begin{tabular}{|c|c|c|c|}
\hline Depth $(\mathrm{Ft})$ & 73 & 66 & 46 \\
\hline Sample R5508 & $\mathrm{C}$ & $\mathrm{B}$ & \\
\hline Series & \multicolumn{3}{|c|}{ Paleocene } \\
\hline \multicolumn{4}{|l|}{ Taxa } \\
\hline Andalusiella polymorpha (Malloy 1972) Lentin \& Williams 1977 & $\mathrm{x}$ & . & \\
\hline Andalusiella sp. aff. A. polymorpha of Edwards (1980) & & & $\mathrm{x}$ \\
\hline Carpatella cornuta Grigorovich 1969 (fragment) & $\mathrm{x}$ & & \\
\hline Cordosphaeridium inodes (Klumpp 1953) Eisenack 1963 & & $\mathrm{x}$ & \\
\hline Damassadinium californicum (Drugg 1967) Fensome et al. 1993 & $\mathrm{x}$ & . & \\
\hline Deflandrea n. sp. aff. D. truncata Eisenack 1938 & ? & 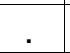 & \\
\hline Fibrocysta Stover \& Evitt 1978 sp. & & $\mathrm{x}$ & \\
\hline Hafniasphaera septata (Cookson \& Eisenack 1967) Hansen 1977 & & $\mathrm{x}$ & \\
\hline Oligosphaeridium complex (White 1842) Davey \& Williams 1966 & & $\mathrm{x}$ & \\
\hline Operculodinium Wall 1967 sp. & & $\mathrm{X}$ & \\
\hline Palaeocystodinium Alberti $1961 \mathrm{sp}$. (almost golzowense) & & . & $\mathrm{x}$ \\
\hline Palaeocystodinium Alberti 1961 sp. (fat) & $\mathrm{x}$ & & \\
\hline Palaeoperidinium pyrophorum (Ehrenberg 1838) Sarjeant 1967 & & $\mathrm{x}$ & \\
\hline Phelodinium sp. of Edwards (1989) & & $\mathrm{x}$ & $\mathrm{x}$ \\
\hline Phelodinium Stover \& Evitt 1978 spp. & & $\mathrm{x}$ & \\
\hline Spiniferites Mantell 1850 spp. & & $\mathrm{x}$ & $\mathrm{x}$ \\
\hline Tanyosphaeridium xanthiopyxides (Wetzel 1933) Stover \& Evitt 1978 & & $\mathrm{x}$ & \\
\hline Tectatodinium rugulatum (Hansen 1977) McMinn 1988 (sensu amplo) & $\mathrm{x}$ & . & \\
\hline Тепиа sp. cf T. formosa of Kurita and McIntyre (1995) & $\mathrm{x}$ & & \\
\hline Trigonipyxidia ginella (Cookson \& Eisenack 1961) Downie \& Sarjeant 1965 & & . & $\mathrm{x}$ \\
\hline Trithyroidinium sp. & $\mathrm{x}$ & & \\
\hline miscellaneous areoligeracean forms & & $\mathrm{x}$ & \\
\hline small peridiniacean forms & $\mathrm{x}$ & $\mathrm{x}$ & $\mathrm{x}$ \\
\hline $\mathrm{X}=$ present; .=not present; ?=questionable & & & \\
\hline & & & \\
\hline & & & \\
\hline
\end{tabular}




\begin{tabular}{|l|c|c|c|}
\hline \multicolumn{1}{|c|}{$\begin{array}{r}\text { Depth in ft } \\
\text { Sample }\end{array}$} & R5508C & R5508B & R5508A \\
\hline \multicolumn{1}{|c|}{ Taxa; age range } & $\begin{array}{c}\text { Danian; } \\
\text { younger } \\
\text { than type } \\
\text { Rhems? }\end{array}$ & $\begin{array}{c}\text { NP5 and } \\
\text { younger }\end{array}$ & $\begin{array}{c}\text { No useful } \\
\text { species }\end{array}$ \\
\hline Alnus sp. & & & \\
\hline Bombacacidites paulus &. &. & $\mathrm{X}$ \\
\hline Bombacacides reticulatus &. &. & $\mathrm{X}$ \\
\hline Diporate, tiny & $\mathrm{X}$ & $\mathrm{X}$ & $\mathrm{X}$ \\
\hline Favitricolporites baculoferus & $\mathrm{X}$ &. &. \\
\hline Lanagiopollis cribellatus & $\mathrm{X}$ & $\mathrm{X}$ &. \\
\hline Momipites coryloides/microfoveolatus & $\mathrm{X}$ &. &. \\
\hline Momipites tenuipolus group & $\mathrm{X}$ & $\mathrm{X}$ & $\mathrm{X}$ \\
\hline Nudopollis terminalis & $\mathrm{X}$ & $\mathrm{X}$ &. \\
\hline Nyssapollenites paleocenicus & $\mathrm{X}$ &. & $\mathrm{X}$ \\
\hline Plicatopollis triradiatus &. &. & $\mathrm{X}$ \\
\hline Pseudoplicapollis limitatus & $\mathrm{X}$ & $\mathrm{X}$ &. \\
\hline Pseudoplicapollis serenus & $\mathrm{X}$ &. & $\mathrm{X}$ \\
\hline Subtriporopollenites nanus NP5 and younger &. & $\mathrm{X}$ &. \\
\hline Thomsonipollis magnificus &. & $\mathrm{X}$ &. \\
\hline Tricolpites asper & $\mathrm{X}$ & $\mathrm{X}$ &. \\
\hline Trudopollis spp., including T. plenus &. & $\mathrm{X}$ & $\mathrm{X}$ \\
\hline \multicolumn{2}{|r|}{} & & \\
\hline Figure 24. Distribution of pollen taxa in Paleocene samples from the USGS/DNR & \\
Swansea corehole (LEX-844), Lexington County, South Carolina. & & \\
\hline
\end{tabular}




\begin{tabular}{|c|c|c|c|c|c|}
\hline Depth $(\mathrm{Ft})$ & $\begin{array}{l}\tilde{0} \\
\infty\end{array}$ & $\begin{array}{l}N \\
0 \\
0 \\
0 \\
1 \\
1 \\
0 \\
0 \\
\end{array}$ & $\begin{array}{l}\text { N } \\
\infty \\
0 \\
1 \\
\sim \\
D \\
0 \\
\end{array}$ & $\underset{N}{N}$ & N \\
\hline Sample R5317 & $\mathrm{BE}$ & $\mathrm{V}$ & $\mathrm{W}$ & & $\overline{B C}$ \\
\hline Series & \multicolumn{5}{|c|}{ Paleocene } \\
\hline Subseries & \multicolumn{5}{|c|}{ Lower } \\
\hline Taxon & & & & & \\
\hline ?Andalusiella rhombohedra of Edwards and others (1984) & $\mathrm{X}$ & $\mathrm{X}$ & . & $\mathrm{X}$ & \\
\hline Achomosphaera alcicornu (Eisenack 1954) Davey \& Williams 1966 & . & . & . & . & $\mathrm{P}$ \\
\hline Andalusiella polymorpha (Malloy 1972) Lentin \& Williams 1977 & & . & . & $\mathrm{X}$ & \\
\hline Andalusiella sp. aff. A. polymorpha of Edwards (1980) & ? & . & . & . & \\
\hline Areoligera volata Drugg 1967 & $\mathrm{X}$ & . & . & $\mathrm{X}$ & $\mathrm{P}$ \\
\hline Carpatella cornuta Grigorovich 1969 & . & . & $\mathrm{X}$ & $\mathrm{X}$ & $\mathrm{P}$ \\
\hline Catillopsis abdita Drugg 1970 & & . & . & . & $\mathrm{P}$ \\
\hline Cordosphaeridium fibrospinosum Davey \& Williams 1966 & $\mathrm{X}$ & $\mathrm{X}$ & $\mathrm{X}$ & . & . \\
\hline Cordosphaeridium Eisenack 1963 spp. & & . & . & $\mathrm{X}$ & $\mathrm{P}$ \\
\hline Cribroperidinium Neale \& Sarjeant 1962 sp. & $\mathrm{X}$ & . & . & . & . \\
\hline Cyclapophysis monmouthensis Benson 1976 & $\mathrm{X}$ & . & . & . & . \\
\hline Damassadinium californicum (Drugg 1967) Fensome et al. 1993 & & . & $\mathrm{X}$ & $\mathrm{X}$ & $\mathrm{P}$ \\
\hline Deflandrea cf. D. diebelii Alberti of Drugg (1967) & ? & . & $\mathrm{X}$ & ? & ? \\
\hline Deflandrea n. sp. aff. D. truncata Eisenack 1938 & $\mathrm{X}$ & $\mathrm{X}$ & . & $\mathrm{X}$ & $\mathrm{P}$ \\
\hline Diphyes colligerum (Deflandre \& Cookson 1955) Cookson 1965 & . & . & . & $\mathrm{X}$ & $\mathrm{P}$ \\
\hline Exochosphaeridium Davey et al. 1966 sp. & & . & $\mathrm{X}$ & $\mathrm{X}$ & . \\
\hline Fibrocysta Stover \& Evitt 1978 spp. & $\mathrm{X}$ & . & . & . & . \\
\hline Hafniasphaera Hansen 1977 spp. & $\mathrm{X}$ & . & $\mathrm{X}$ & . & \\
\hline Hystrichosphaeridium Deflandre 1937 spp. & $\mathrm{X}$ & . & . & $\mathrm{X}$ & . \\
\hline Ifecysta Jan du Chêne \& Adediran 1985 sp. & $\mathrm{X}$ & . & . & . & \\
\hline Impagidinium Stover \& Evitt 1978 sp. & & . & . & . & $\mathrm{P}$ \\
\hline Operculodinium Wall 1967 spp. & $\mathrm{X}$ & $\mathrm{X}$ & . & $\mathrm{x}$ & $\mathrm{P}$ \\
\hline Palaeocystodinium Alberti 1961 (fat) & . & . & . & $\mathrm{X}$ & $\mathrm{P}$ \\
\hline Palaeocystodinium golzowense Alberti 1961 & . & $\mathrm{X}$ & . & . & $\mathrm{P}$ \\
\hline Pentadinium group & & . & $\mathrm{C}$ & . & \\
\hline Phelodinium magnificum (Stanley 1965) Stover \& Evitt 1978 & $\mathrm{X}$ & . & $\mathrm{X}$ & $\mathrm{X}$ & $\mathrm{P}$ \\
\hline Polyspheridium zoharyi (Rossignol 1962) Bujak et al. 1980 & & . & & & $\mathrm{P}$ \\
\hline Senoniasphaera inornata (Drugg 1970) Stover \& Evitt 1978 & & . & $\mathrm{X}$ & $\mathrm{X}$ & $\mathrm{P}$ \\
\hline Spinidinium pulchrum (Benson 1976) Lentin \& Williams 1977 & $\mathrm{X}$ & $\mathrm{X}$ & $\mathrm{X}$ & $?$ & ? \\
\hline Spinidinium Cookson \& Eisenack 1962 spp. & $\mathrm{X}$ & $\mathrm{X}$ & $\mathrm{X}$ & $\mathrm{X}$ & $\mathrm{P}$ \\
\hline Spiniferites Mantell 1850 spp. & $\mathrm{X}$ & $\mathrm{X}$ & $\mathrm{x}$ & $\mathrm{X}$ & $\mathrm{P}$ \\
\hline Tectatodinium rugulatum (Hansen 1977) McMinn 1988 & $\mathrm{X}$ & . & $\mathrm{X}$ & & $\mathrm{cfF}$ \\
\hline Tenua sp. cf. T. formosa of Kurita and McIntyre (1995) & & . & $\mathrm{X}$ & $\mathrm{X}$ & $\mathrm{P}$ \\
\hline Turbiosphaera Archangelsky 1969 sp. & $\mathrm{X}$ & $\mathrm{X}$ & . & & \\
\hline miscellaneous areoligeracean forms & $\mathrm{X}$ & $\mathrm{X}$ & . & $\mathrm{X}$ & $\mathrm{P}$ \\
\hline small peridiniacean forms & $\mathrm{X}$ & $\mathrm{X}$ & $\mathrm{X}$ & $\mathrm{X}$ & $\mathrm{P}$ \\
\hline $\begin{array}{l}\mathrm{X}=\text { present; } .=\text { not present; ?=questionable; } \mathrm{P}=\text { present in the sample, but not } \\
\text { necessarily in place stratigraphically. }\end{array}$ & & & & & \\
\hline Figure 25. Distribution of dinocyst taxa in Paleocene samples & & & & & \\
\hline School No. 1 corehole (ORG-393), Orangeburg County, South Carolina. & & & & & \\
\hline
\end{tabular}




\begin{tabular}{|c|c|c|c|c|}
\hline Depth (Ft) & 298 & $289.0-289.3$ & $278.0-278.3$ & 274.0 \\
\hline Sample & R5317BE & R5317V & R5317W & R5317BD \\
\hline $\begin{array}{r}\text { Age, correlation, } \\
\text { or NP zone } \\
\text { equivalent }\end{array}$ & Danian & \begin{tabular}{|c|} 
Type Rhems \\
Formation \\
or slightly \\
younger* $^{\star}$
\end{tabular} & NP 3-5 & Rare pollen \\
\hline \multicolumn{5}{|l|}{ Pollen Taxa And Known Ranges } \\
\hline Bombacacidites nacimientoensis & $\mathrm{X}$ & . & $\mathrm{X}$ & . \\
\hline Bombacacides reticulatus & $\mathrm{X}$ & $\mathrm{X}$ & $\mathrm{X}$ & $\mathrm{X}$ \\
\hline Caryapollenites prodromus group & . & $?$ & $\mathrm{X}$ & . \\
\hline Choanopollenites alabamicus NP8 \& older & . & $\mathrm{X}$ & $\mathrm{X}$ & . \\
\hline Diporate, tiny & $\mathrm{X}$ & $\mathrm{X}$ & . & . \\
\hline Favitricolporites baculoferus & . & . & $\mathrm{X}$ & . \\
\hline Holkopollenites chemardensis & . & . & $\mathrm{X}$ & . \\
\hline Interpollis paleocenicus & . & . & $\mathrm{X}$ & . \\
\hline Intratriporopollenites pseudinstructus & $?$ & . & . & . \\
\hline Milfordia minima & . & $\mathrm{X}$ & . & . \\
\hline Momipites coryloides/microfoveolatus & $\mathrm{X}$ & & $\mathrm{X}$ & $\mathrm{X}$ \\
\hline Momipites dilatus & . & $\mathrm{X}$ & . & . \\
\hline Momipites dilatus/flexus & . & $\mathrm{X}$ & . & . \\
\hline Momipites tenuipolus group & $\mathrm{X}$ & $\mathrm{X}$ & $\mathrm{X}$ & $\mathrm{X}$ \\
\hline Nudopollis terminalis & . & & $\mathrm{X}$ & . \\
\hline Nudopollis thiergartii & . & $\mathrm{X}$ & . & . \\
\hline Nyssapollenites paleocenicus - type Rhems & . & $\mathrm{X}$ & . & . \\
\hline Osculapollis ? colporatus NP 3-5 & . & $\mathrm{X}$ & $\mathrm{X}$ & . \\
\hline Pseudoplicapollis serenus Up Cret.-top of Danian & $\mathrm{X}$ & $\mathrm{X}$ & . & . \\
\hline Retitrescolpites anguloluminosus & $?$ & . & . & . \\
\hline Sparganiaceaepollenites sp. & . & . & $\mathrm{X}$ & . \\
\hline Subtriporopollenites anulatus & . & . & $\mathrm{X}$ & . \\
\hline Tricolpites asper & . & . & $\mathrm{X}$ & . \\
\hline Tricolpites microreticulatus & . & $\mathrm{X}$ & . & . \\
\hline * The Rhems Formation is mainly or entirely Danian in age. & & & & \\
\hline
\end{tabular}




\begin{tabular}{|c|c|c|}
\hline Depth (feet) & $\begin{array}{l}\text { N } \\
0 \\
0 \\
\stackrel{1}{0} \\
\mathbb{0} \\
\infty\end{array}$ & 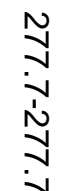 \\
\hline Sample R5979 & $E$ & $\mathrm{~J}$ \\
\hline $\begin{array}{r}\text { Series } \\
\text { Subseries }\end{array}$ & \multirow{2}{*}{\multicolumn{2}{|c|}{$\begin{array}{c}\text { Paleocene } \\
\text { Lower }\end{array}$}} \\
\hline Subseries & & \\
\hline \multicolumn{3}{|l|}{ Taxa } \\
\hline Andalusiella polymorpha (Malloy 1972) Lentin \& Williams 1977 & $\mathrm{X}$ & \\
\hline Andalusiella Riegel 1974 spp. & & $\mathrm{X}$ \\
\hline Areoligera volata Drugg 1967 & ? & $\mathrm{X}$ \\
\hline Carpatella cornuta Grigorovich 1969 & & $\mathrm{X}$ \\
\hline Catillopsis Drugg 1970 sp. & & $\mathrm{cf}$ \\
\hline Cordosphaeridium fibrospinosum Davey \& Williams 1966 & $\mathrm{X}$ & $\mathrm{X}$ \\
\hline Cribroperidinium Neale \& Sarjeant 1962 spp. & $\mathrm{X}$ & \\
\hline Damassadinium californicum (Drugg 1967) Fensome et al. 1993 & & $\mathrm{X}$ \\
\hline Deflandrea cf. D. diebelii Alberti of Drugg (1967) & ? & $\mathrm{X}$ \\
\hline Deflandrea n. sp. aff. D. truncata Eisenack 1938 & $\mathrm{X}$ & $\mathrm{X}$ \\
\hline Diphyes colligerum (Deflandre \& Cookson 1955) Cookson 1965 & $\mathrm{X}$ & $\mathrm{X}$ \\
\hline Exochosphaeridium Davey et al. $1966 \mathrm{sp}$. & & $\mathrm{X}$ \\
\hline Fibrocysta Stover \& Evitt 1978 spp. & $\mathrm{X}$ & \\
\hline Hafniasphaera Hansen 1977 spp. & $\mathrm{X}$ & \\
\hline Hystrichokolpoma unispinum Williams \& Downie 1966 & & $\mathrm{X}$ \\
\hline Hystrichosphaeridium tubiferum (Ehrenberg 1838) Deflandre 1937 & & $\mathrm{X}$ \\
\hline Phelodinium magnificum (Stanley 1965) Stover \& Evitt 1978 & & $\mathrm{X}$ \\
\hline Phelodinium Stover \& Evitt 1978 sp. & $\mathrm{X}$ & \\
\hline Senoniasphaera inornata (Drugg 1970) Stover \& Evitt 1978 & & $\mathrm{X}$ \\
\hline Spinidinium pulchrum (Benson 1976) Lentin \& Williams 1977 & & $\mathrm{X}$ \\
\hline Spinidinium Cookson \& Eisenack 1962 spp. & $\mathrm{X}$ & $\mathrm{X}$ \\
\hline Spiniferites Mantell 1850 spp. & $\mathrm{X}$ & $\mathrm{X}$ \\
\hline Tectatodinium rugulatum (Hansen 1977) McMinn 1988 sensu amplo & $\mathrm{X}$ & $\mathrm{X}$ \\
\hline Tenua sp. cf. T. formosa of Kurita and McIntyre (1995) & & $\mathrm{X}$ \\
\hline miscellaneous areoligeracean forms & & $\mathrm{X}$ \\
\hline small peridiniacean forms & $\mathrm{X}$ & $\mathrm{x}$ \\
\hline $\mathrm{X}=$ present; $=$ not present; ?=questionable; $\mathrm{cf}=$ comparable & & \\
\hline & & \\
\hline
\end{tabular}

Figure 27. Distribution of dinocyst taxa in Paleocene samples from the USGS/DNR Clark School No. 2 corehole (ORG-430), Orangeburg County, South Carolina. 


\begin{tabular}{|c|c|c|c|c|c|c|c|}
\hline Depth in $\mathrm{ft}$ & 298.0-298.1 & $295.3-295.5$ & 295.5-295.6 & 291.4-291.5 & $284.9-285.0$ & 281.3-281.4 & 277.7-277.8 \\
\hline Sample & R5979E & \begin{tabular}{|l|} 
R5979F \\
\end{tabular} & R5979CE & R5979CD & R5979CC & R5979CB & R5979CA \\
\hline Age or correlation & $\begin{array}{l}\text { Type } \\
\text { Rhems } \\
\text { Formation }\end{array}$ & $\begin{array}{l}\text { Type } \\
\text { Rhems } \\
\text { Formation }\end{array}$ & $\begin{array}{l}\text { Type } \\
\text { Rhems } \\
\text { Formation }\end{array}$ & $\begin{array}{c}\text { Rare pollen } \\
\text { grains }\end{array}$ & $\begin{array}{c}\text { Upper type } \\
\text { Rhems Fm. } \\
\text { or slightly } \\
\text { younger }\end{array}$ & $\begin{array}{c}\text { Sparse, } \\
\text { useless } \\
\text { pollen } \\
\text { grains }\end{array}$ & $\begin{array}{c}\text { Sparse, } \\
\text { useless } \\
\text { pollen } \\
\text { grains }\end{array}$ \\
\hline \multicolumn{8}{|l|}{ Sample } \\
\hline \multicolumn{8}{|l|}{ Taxa } \\
\hline Bombacacides reticulatus & $\mathrm{X}$ & $\mathrm{X}$ & . & . & $\mathrm{X}$ & . & . \\
\hline Diporate, tiny & . & . & . & . & $\mathrm{X}$ & . & . \\
\hline Momipites coryloides/microfoveolatus & . & $\mathrm{X}$ & . & . & . & . & . \\
\hline Momipites dilatus & . & . & . & . & $\mathrm{X}$ & . & . \\
\hline Momipites flexus & . & . & . & . & $\mathrm{X}$ & . & . \\
\hline Momipites tenuipolus group & 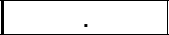 & . & $\mathrm{X}$ & . & $x$ & . & . \\
\hline Nyssapollenites paleocenicus & $\mathrm{X}$ & & $\mathrm{X}$ & . & $x$ & . & . \\
\hline Sparganiaceaepollenites sp. & $\mathrm{X}$ & $\mathrm{X}$ & $\mathrm{X}$ & . & . & . & . \\
\hline Tricolpites microreticulatus & $\mathrm{X}$ & $\mathrm{X}$ & . & . & . & . & . \\
\hline & & & & & & & \\
\hline $\begin{array}{l}\text { Figure 28. Distribution of pollen taxa } \\
\text { Orangeburg County, South Carolina. }\end{array}$ & in Paleocene & omolo fo & tho IISG & S & $\mathrm{abe} \mathrm{No}$ ? & & \\
\hline
\end{tabular}

